\title{
Accelerator-based Nuclear Techniques for Processing and Characterization of Oxide Semiconductors for Solar Energy Conversion
}

Zeljko Pastuovic ${ }^{1}$, Mihail lonescu ${ }^{* 1}$, Ettore Vittone ${ }^{2}$, Ivana Capan ${ }^{3}$

${ }^{1}$ Australian Nuclear Science and Technology Organisation, Australia

2University of Torino, Italy

${ }^{3}$ Rudjer Boskovic Institute, Croatia

Corresponding author: zkp@ansto.gov.au

Keywords: accelerator-based nuclear techniques; defects in semiconductors; ion implantation

Accelerator-based nuclear techniques are an important tool for the modification and characterization of surfaces in general, down to a depth of around one micrometer. For oxide semiconductors used in solar energy conversion, the surface plays a critical role in facilitating the use of solar photon energy to obtain hydrogen via spontaneous water oxidation. For such a process, the required surface properties are complex and include specific chemical composition, as well as the defect composition, and both of these characteristics may be augmented using accelerator-based nuclear techniques. The targeted modification of surfaces makes use of ion implantation for changing the chemical composition, and ion irradiation for changing the defect structure. The defect formation is a very complex process, and in this work we placed more emphasis on this aspect. We attempted to present the defect formation under the irradiation of ion beams at the two extremes: formation of extensive and large-scale cluster defects; and formation of small-scale point defects. In addition, we review the main characterization techniques based on ion beams, with examples from work carried out on semiconductors and oxide semiconductors. 


\section{Introduction}

Oxide semiconductors are promising candidates for the conversion of solar energy into the chemical energy, primarily for their resistance to water corrosion. The performance in partial or total oxidation of water depends on the surface, the near-surface and the bulk properties [1]. Currently this performance is low, due to an efficiency of around $2 \%$, and a viable commercial product would require efficiency above $10 \%$. This may be achieved by maximizing the key performance-related properties (KPPs), as defined in the previous chapters, using a combination of surface, near-surface and bulk techniques to modify them in a systematic manner.

The present chapter introduces the accelerator-based nuclear techniques and their application to the modification and characterization of semiconductors. It is believed that the low level defects induced in semiconductor materials and junctions are of interest for photo-catalysis, and therefore this aspect will be treated in more detail.

\section{Ion Accelerators}

Ion accelerators are typically electrostatic devices, where ions are produced in an ion source, and mass analyzed by a magnetic, electric or a combination of magnetic and electric fields. The selected ion is then accelerated over a gap with electric potential difference in order to acquire the necessary kinetic energy. This can be a single stage gap or a stack of multiple gaps, each at an appropriate electric potential difference, forming an accelerating column. At one end of the column and electrically isolated, there is a metallic dome which is charged to the appropriate accelerating voltage, which is distributed to all accelerating gaps using appropriate resistors. The maximum attainable voltage on the dome is a main characteristic of an ion accelerator, and is a measure of the maximum attainable kinetic energy for accelerated ions. The main parameters of the ion beam produced by an electrostatic accelerator are the mas of ion, the kinetic energy of ion and the ion beam current.

Ion sources can produce negative or positive ions from solid or gas primary feed. The choice is dictated by the type of ion electrostatic accelerator, which may have one or two accelerating columns. For a single-column accelerator the ion sources may produce either positive or negative ions. However, for double column accelerators (tandems), the ion sources associated with them must produce negative ions only, the reason being the necessity to exchange the charge of accelerated ions when passing from the first accelerating column to the second accelerating column, and the charge exchange is easier from a negative to a positive charge then the other way around.

In the process of charge exchange, the negative ion can produce a number of positive ions, with different charges, and different charge distributions, each of which will be accelerated at different energies in the second column. Thus, at the exit of the second column, the ion beam represents a bunch of various beams with different energies and beam currents. The selection of the appropriate beam is then necessary, and it is performed with the aid of magnetic, electric or a combination of magnetic and electric fields. At the end of this mass and energy analysis process the beam is mono energetic and is ready to interact with the target. In order to achieve this, a typical ion accelerator system has a number of experimental beamlines, configured for specific experimental conditions.

A schematic of an ion accelerator system with a visual depiction of the above-mentioned stages of ion acceleration is shown in Figure 1.

The experimental beamlines terminate in a specific experimental vacuum chamber, which house the sample, the sample manipulation system, the detection system and other auxiliary systems, such as microscopes, hot/cold stages, etc. A more detailed 
description of one experimental chambers is shown in Figure 2, which represents a micro-beam setup. In this case, the ion beam is focused down

to around $1 \mu \mathrm{m}$ diameter, using a set of magnetic quadrupoles, just before it enters in the vacuum chamber. This arrangement is called an ion microprobe, and it works in a similar fashion as an electron microscope, using ions instead of electrons, which are rastered on the sample surface.

\section{Accelerator Techniques}

There are two broad classes of accelerator techniques, grouped under the name of accelerator mass spectrometry (AMS) and ion beam analysis (IBA). AMS is using the electrostatic accelerator as a mass spectrometer, and measures ratios of specific isotopes. It has a very high resolution $\left(1: 10^{15}\right)$, but a poor dynamic range being capable to measure only one isotope ratio at a time, with the destruction of the sample. IBA is the group of techniques which encompasses anything else which is not AMS, such as ion implantation, irradiation, study of nuclear reactions, depth profiling, thin film analysis, neutron production, etc.

\section{Ion Implantation}

Implantation is the process of bombarding the solid with specific ions, which possess the kinetic energy that is required to penetrate the surface and come to rest at a particular depth inside the target (crystalline or amorphous). In this process, the original chemical composition is changed and some atoms of the crystal lattice will be displaced by recoil from their equilibrium position. Therefore, implantation cannot be considered as doping in terms of the formation of solid solutions that can be obtained using either solid state reactions or soft chemistry routes. As a consequence, implantation process results in the formation of systems that are remote from thermodynamic equilibrium. The properties of implanted solids depend on the selected ion and its energy used for implantation. As a result, thermodynamics cannot be applied to describe the properties of solids formed by ion implantation. However, the post-implantation annealing may be used to gradually transfer the system from its non-equilibrium state to a system that can be equilibrated at the local atomic level. In this process, the implanted ions may diffuse inside the target or may diffuse and coalesce into nanoparticles, under the target surface or on the surface [2]. Therefore the post implantation annealing process may be used in the formation of systems that are partially equilibrated. The related systems exhibit the properties that represent the characteristics related to both implantation and chemical doping.

This technique has the capability to implant ions of most of the elements in the Periodic Table at both low energy (few keV) and medium energy (few MeV). It is a very versatile and powerful tool for surface and near-surface modifications of materials mostly because this is a non-equilibrium technique, and thus it can be used to implant any ion in any matrix. The choice of energy is a function of the mass of implanted ion, the required implantation depth and the target properties. For a particular ion, the lower the energy, the shallower the implantation depth, but below a specific threshold energy, the ion is no longer able to penetrate the energy barrier of the target surface, and comes to rest on the target surface. For some ion-energy-target combinations this result in sputtering of target atoms, associated with surface damage. If the ion energy is further reduced, it may results in a deposition process. At the higher end of implantation energy, sputtering of the target surface may be the dominant process. Implantation offers precise control of composition and when it is carried out through masking or with a focused ion beam, it also offers precise control of location on the surface of the sample.

A variation of this technique called "Micro-Implantation" is achieved when the ion beam is focused down to a micron-size spot. If the ion current is sufficiently high, for some 
target materials this leads to micro-implantation or micro-sputtering and can be used to create implanted or damaged islands, or to "write" complex shapes on the surfaces [3].

There are numerous examples of the use of ion implantation for materials, such as semiconductors, corrosion resistant materials, polymers, glasses, etc. In the case of oxide semiconductors, implantation can offer the capability to change the surface composition and defects. In particular for $\mathrm{TiO}_{2} \mathrm{POS}$, shallow implantation is one of the few ways to control the surface and sub-surface doping [4]. This leads to controlled changes in chemical composition at the surface and sub-surface regions, with the direct effect on electronic structure modifications. In addition, surface and sub- surface doping can lead to nano-meter cluster formation on the surfaces and the enhancement of plasmonic effects.

In many cases, photo-catalytic materials obtained by ion implantation are called second generation photo-catalytic materials. In the case of thin films of polycrystalline $\mathrm{TiO}_{2}$ made by ICB (ion cluster beam) on glass [5], and implanted by $\mathrm{V}^{+}, \mathrm{Cr}^{+}, \mathrm{Mn}^{+}$, $\mathrm{Fe}^{+}$, at $150 \mathrm{keV}$ and a dose of $10^{16}$ ions $/ \mathrm{cm}^{2}$ and $6 \times 10^{16}$ ions $/ \mathrm{cm}^{2}$, the UV-VIS transmittance show a shift towards higher wavelength with the increase of implanted dose. XAFS indicated the replacement of $\mathrm{Ti}^{4+}$ by $\mathrm{V}^{3+}, \mathrm{V}^{4+}$, and ab-initio DFT calculation showed a mixture of $\mathrm{Ti}(\mathrm{d}), \mathrm{V}(\mathrm{d})$ and $\mathrm{O}(\mathrm{d})$ orbitals, which suggest an explanation for the red-shift [6].

In a similar study [7], $\mathrm{Cr}^{+}$and $\mathrm{V}^{+}$ions implanted in $\mathrm{TiO}_{2}$ thin film show 2 to 3 times higher photocatalitic activity than non-implanted material, but the film thickness and the substrate temperature during the film deposition played a critical role in UV-VIS absorption. In the case of $\mathrm{Cr}$ implantation, it was showed that it also decreased the band gap (BG) and $\mathrm{TiO}_{2}$ absorbed visible light, suggesting that implanted $\mathrm{Cr}$ does not form recombination centres like the $\mathrm{Cr}$ doped chemically. Also, $\mathrm{V}, \mathrm{Mn}, \mathrm{Ni}, \mathrm{Ar}, \mathrm{Mg}, \mathrm{Ti}$, and $\mathrm{Fe}$ were implanted in $\mathrm{TiO}_{2}$, and the red shift achieved increased in the following order: $\mathrm{V}>$ $\mathrm{Cr}>\mathrm{Mn}>\mathrm{Fe}>\mathrm{Ni}$, but required annealing at 723-823K in $\mathrm{O}_{2}$ for achieving it. Molecular orbital calculations showed that $\mathrm{V}^{3+}, \mathrm{V}^{4+}, \mathrm{V}^{5+}$ substitute octahedral $\mathrm{Ti}^{4+}$, and therefore $\mathrm{Ti}(\mathrm{d})$ and implanted ion-metal (d) orbital mixing led to BG narrowing. Preliminary investigations show that ion implantation is advantageous as compared to other semiconductors and dye sensitised materials for which electron $\mathrm{e}^{-}$mediator and sacrificial agents are necessary, which is not the case for implantation.

In the same study [7], polycrystalline $\mathrm{TiO}_{2}$ and $\mathrm{WO}_{3}$, loaded with metals ( $\mathrm{Pt}, \mathrm{Au}, \mathrm{Pd}, \mathrm{Rh}$, $\mathrm{Ni}, \mathrm{Cu}, \mathrm{Ag}$ ) and/or doped with metal ions (Fe, Mo, Ru, Os, Re, V, Rh) by implantation, increase the photocatalitic activity. Also, out of (La, Ce, Er, Pr, Gd, Nd, Sm), Gd was the best in increasing the photocatalitic activity, and doping with $\mathrm{Be}$ at near the surface is beneficial, but when Be was implanted deeper the benefit is lost. In the case of $\mathrm{WO}_{3}$, doping with $\mathrm{Ni}$ achieved better photocatalitic activity than doping with $\mathrm{Fe}, \mathrm{Co}, \mathrm{Cu}, \mathrm{Zn}$, $\mathrm{Mn}$, but it was shown that $\mathrm{Cu}, \mathrm{Mn}$ and $\mathrm{Fe}$ can trap both $\mathrm{e}^{-}$and $\mathrm{h}^{+}$. Anion doping of polycrystalline $\mathrm{TiO}_{2}$ with $\mathrm{N}, \mathrm{C}, \mathrm{F}, \mathrm{S}$ can also narrow the $\mathrm{BG}$, and is less likely to form recombination centres (unlike metal ions), and so be more effective. Doping by implantation with $C$ and $P$ were less effective as the introduced states were too deep and photo-generated charge carriers were difficult to be transferred to the surface. Sulphur was showed to provide $S 3 p$ states mixed with the valence band (VB) of $\mathrm{TiO}_{2}$ and increase the width of VB, by shifting upwards with no change on the width of conduction band $(\mathrm{CB})$, and consequently narrowing the BG. Similar behaviour was observed for $\mathrm{N}$-doping.

Ion implantation is one of the most promising technologies for improving $\mathrm{TiO}_{2}$ properties because it allows for a high impurity filling factor, beyond the equilibrium limit of impurity solubility, and also provide control on the nanoparticles formed at different depth levels. Anatase phase appears to be better than rutile because of native higher band bending, deeper under the surface, and with a steeper gradient, enhancing the surface holes trapping [8]. In rutile, the $\mathrm{e}^{-/} \mathrm{h}^{+}$recombination is pronounced in the bulk regions, so only 
a smaller fraction of holes are trapped and transferred to the surface. The low ion implantation dose of less than $10^{14}$ ions $/ \mathrm{cm}^{2}$ results in well dispersed implanted ions. Between $10^{15}$ ions $/ \mathrm{cm}^{2}$ and $10^{17}$ ions $/ \mathrm{cm}^{2}$, the implanted ions exceed the solubility limit and form nano-particles (NP) by nucleation and growth. Low implantation energies between 10-300keV is of higher interest because it results in the implanted species being in the proximity of the surface. Implantation with $\mathrm{Fe}, \mathrm{V}, \mathrm{Cr}, \mathrm{Mn}, \mathrm{Co}, \mathrm{Ni}, \mathrm{Cu}$ was effective in the red shift of $\mathrm{TiO}_{2}$, whilst $\mathrm{Ti}, \mathrm{Ar}$, Na was not. From EXAFS, $\mathrm{Fe}^{3+}$ appears to replace $\mathrm{Ti}^{4+}$ and the same for $\mathrm{V}$ and $\mathrm{Cr}$. Also implanted $\mathrm{Nb}$ and $\mathrm{Sn}$ are effective for increasing the matrix conductivity, in particular $\mathrm{Sn}$, which is substitutional up to $\sim 1$ at\%, when increases conductivity very high $\sigma=5-30 / \Omega \cdot \mathrm{cm}$, compared to typical $\sigma=1 / \Omega \cdot \mathrm{cm}$, obtained by conventional doping. Implantation with $\mathrm{Cr}$ at room temperature (RT) increases $\sigma$ from $10^{-13} / \Omega \cdot \mathrm{cm}$ to $10^{-2} / \Omega \cdot \mathrm{cm}[7]$.

Synthesis of metal nanoparticles (MNP) for optic and magnetic properties by ion implantation is very attractive because the MNP can be well controlled to any dimension and structure. $\mathrm{Au}, \mathrm{Ag}$ and $\mathrm{Cu}$ show strong surface plasmon resonance (SPR) in visible or near IR range for $\mathrm{Au}$ implanted at $2 \mathrm{MeV}$ in $\mathrm{TiO}_{2}$ at an angle of $60^{\circ}$, at $\mathrm{RT}$. Also $\mathrm{Ag}$ implanted at $40 \mathrm{keV}$ and $10^{15}-10^{16} \mathrm{ions} / \mathrm{cm}^{2}$ in $\mathrm{TiO}_{2}$ shows poly-colour photochromism and antibacterial properties [8].

Thin films of $\mathrm{TiO}_{2}$ grown by reactive magnetron sputtering to $75 \mathrm{~nm}$ thick, were doped by ion implantation with $\mathrm{Au}$ at $150 \mathrm{keV}$, and a dose between $5 \times 10^{15}-10^{17}$ ions $/ \mathrm{cm}^{2}$. At the lower fluence of $5 \times 10^{16}$ ions $/ \mathrm{cm}^{2}$ Au nanoclusters $3-5 \mathrm{~nm}$ (by XRD) start to form in the film at $\mathrm{RT}$, and during annealing at $350^{\circ} \mathrm{C}$ in reduced atmosphere, the nanocluster start to coalesce, following an Oswald ripening mechanism. Following annealing at $600^{\circ} \mathrm{C}$, the precipitate dimensions reach $40 \mathrm{~nm}$ (all by in-situ XRD), and annealing above $700^{\circ} \mathrm{C}$ promote the formation of two Au-rich regions, at the interface and at the surface, shown by He-RBS [9].

Rutile (110) single crystals were implanted with $\mathrm{Er}$ at 40,80, 150, 200, 350keV, at fluences of $10^{14}, 1.5 \times 10^{14}, 2 \times 10^{14}, 1.5 \times 10^{14}$, and $6 \times 10^{14}$ ions $/ \mathrm{cm}^{2}$ respectively. Some samples were annealed in $\mathrm{Ar}$ for $30 \mathrm{~min}$ at 800,900 and $1000^{\circ} \mathrm{C}$. Non-Rutherford Resonant Scattering/Channelling with $3.05 \mathrm{MeV} \mathrm{He}$ along $<110>$, showed no detectable crystal damage, and $\mathrm{Er}$ diffuse a little above $800^{\circ} \mathrm{C}$, suggesting an interstitial position [10].

Irradiation of surfaces with ions can lead to radiation damage and therefore the formation of defects. In well-defined experiments, energy loss mechanisms governing the ion-target interaction are responsible in producing point defects or defect clusters and this defect engineering may be a useful tool in enhancing specific properties of materials, like surface reactivity (including photocatalysis), magnetic flux pinning and others.

In the case of nonstoichiometric oxides, implantation may be used to drive the deviation from stoichiometry in the desired direction. For example, implanting with the host metal cation results in a reduction process, leading to increasing concentration of oxygen vacancies and decreasing the concentration of metal vacancies. However, the implantation process results in destruction of the crystal lattice, therefore, the restoration of the crystalline structure requires post-implantation annealing.

\section{Ion Irradiation}

Ion irradiations is defined as the process of exposure of matter to accelerated ions, with the purpose of creating radiation damage, or trigger specific nuclear reactions, and use the nuclear reaction products as means to achieve other goals, such as the production of neutrons, transmutation doping, and others. Here we treat the high level radiation damage effects, and the low level radiation effects will be treated later. When any ionising radiation (neutron, alpha particles, Gamma-rays etc) interacts with matter, the incident radiation invariably causes damage, and as mentioned earlier, the origin of this damage is the transfer of radiation energy to the target atoms, resulting in permanent 
displacement from their equilibrium lattice positions. It implies that this type of damage is afforded to highly ordered matter (crystalline), and the process is cumulative, decreasing the order (amorphization). The type of damage depends on the incident radiation, predominantly mass and energy, and may result in point defects or clusters of defects, which will be treated later. External particles interacting on the surface of a material, e.g. ion beam, fusion plasma, leads to a defect accumulation process, and it is different from the damage created by a nuclear decaying process, called direct impact damage.

Each of the two is significantly different in their methods of damage production, and in some cases the effects produced in matter. Also, a high level of damage has different macroscopic effects on materials and is treated differently from the low level of damage, shown later in specific cases, taking silicon as example. The direct impact is a form of damage most prevalent in actinide containing materials, e.g. nuclear fuel $\mathrm{UO}_{2}$, and waste forms. It is mentioned here because it may be used to create defects in semiconductor materials by doping with radioactive elements. For example, during a fission event or alpha decay process, two daughter particles are formed. In the case of nuclear fission the two particles can adopt a wide range of isotopes, whereas in alpha decay the daughter particles are an alpha particle $\left({ }^{4} \mathrm{He}\right)$, with the other particle having an atomic mass 4 less than before. The process is schematically represented in Figure 3 , and the full decay chain is described in Table 1.

Using alpha decay as an example, once the decay has occurred there are two particles, one ejected from the other. Due to the conservation of energy and momentum, each ejected particle will have equal and opposite momentum, but different velocities since their masses are significantly different. As the result, the ${ }^{4} \mathrm{He}$ nucleus can move with an energy of $\sim 4 \mathrm{MeV}$, where the recoiling ${ }^{228} \mathrm{Th}$ has an energy range of 80-120 keV. However, while ${ }^{4} \mathrm{He}$ has the higher energy, most of the energy is transferred through electronic interactions (ionisation) and does proportionally little damage. Most of the damage comes from the recoiling ${ }^{228}$ Th nucleus and each decay can cause the movement of thousands of matrix atoms.

During the early stages of damage the amorphous volume grows rapidly and then begins to tail off as it approaches an amorphous fraction of 1 . Mathematically it can be described in the general terms, $f_{a}=1-e^{-0 a D}$, where $f_{a}$ is the fraction of amorphous material, $\sigma_{\mathrm{a}}$ is the damage cross section, and $D$ is the irradiation dose. The $\sigma_{\mathrm{a}}$ is specific to the particle-target atom combination, and it represents the probability for that combination to result in a permanent atom displacement.

The defect accumulation is a process of damage accumulation different to the direct impact process, as amorphisation occurs through the build-up of defects (interstitial and vacancy), which over time result in the loss of long range order. This mechanism is that which occurs in nuclear fusion cores, and from impacting ions, electrons and neutrons. The process of damage can be thought of as follows:

i) Incident ion impacts with 1 atom in the structure, causing displacement of this target atom. It can be any atom within the structure and is known as a "primary knock out atom" (PKA):

ii) Generally both the incident ion and the PKA will continue to move within the structure and impact with other atoms, causing further atoms to be displaced which in turn cause further damage (see Figure 4-a).

iii)This process continues until the entire energy from the incident atom has been transferred to the target material. This process can occur quickly or can take multiple 
impacts, and the process can be elastic or inelastic.

The ion interaction with matter can be simulated with various codes. A good level of accuracy for these processes is provided by the code SRIM [11]. Using this code, an example of damage in $\mathrm{TiO}_{2}$ by $50 \mathrm{keV} \mathrm{Ti}^{+1}$ ions is shown in Figure 4 . On the left (a) it is show a single ion track, where the multiple collisions of the primary $\mathrm{Ti}^{+1}$ ion with the target atoms is visible, with the creation of multiple defects along the ion track. On the right (b) is the formation of damage cascade, following a number of similar ion tracks.

The accumulation of amorphous material is different to the direct impact model. In this case the amorphous fraction remains at a low level, but grows exponentially with dose. Thus the material will rapidly transform from a broadly crystalline material to a largely amorphous material rapidly.

This can be described numerically by the following equation: $f_{a}=e^{\sigma_{a} D}-1$

where $f_{\mathrm{a}}$ is the fraction of amorphous material, $\sigma_{\mathrm{a}}$ is the damage cross section, and $D$ is the dose.

\section{Macroscopic Effects of High Level Radiation Damage}

In general, radiation damage can affect materials in many ways, a few examples are:

1. Expansion of material

2. Cracking

3. Change of properties

In many cases it is found that an amorphous structure occupies more volume, thus a lower density, than the crystalline equivalent, which results in expansion. This by itself can cause problems, particularly for materials which are constrained by containment, e.g. fuels. A secondary factor which can cause problems is the formation of gas bubbles, from ${ }^{4} \mathrm{He}$ and in nuclear fission, $\mathrm{Xe}$ and $\mathrm{Kr}$. These tend to form bubbles within the matrix which in turn cause expansion of the material.

One by-product of sample expansion is the formation of cracks within the material. The primary cause of this is differential expansion which gives rise to stress within the material which is relieved by crack formation. This can occur deep within the material, or in many cases, can occur on the surface. This type of cracking tends to occur when alpha decay introduces stress into the surface, which is minimized by spallation of material.

When cracks form they can be both an advantage and disadvantage. For example, cracks can help remove any gas build up within the samples, but at the same time cracks increase the surface area and increase area for reaction with surroundings, e.g. water in a repository.

One of the effects of radiation damage that is easy to forget is the change in bulk properties that can arise. In many cases metallic samples become brittle and lose their ductility, while in others the material 'crumbles' away. This is one area of materials research where it is vital to model such processes.

A second area of materials research where a change in 'bulk' properties is problematic is the effect of radiation damage on creep. In metallic systems, which have simple crystal structures and in many cases multiple slip planes, radiation damage can induce creep i.e. the metallic sample can move during irradiation by slippage.

Ion beam radiation damage effects can be modelled with ion beams in two ways:

i) In-situ - generally when an ion beam is coupled to an electron microscope or other 
device and the material evaluated during irradiation, without sample removal.

ii) Ex-situ - this is where the material is irradiated, examined separately, with further irradiations and analysis where necessary.

Both techniques have their advantages and disadvantages, for example with in-situ samples within a TEM, the sample has to be thin enough for electrons to pass through, this results in a sample which may not model exactly the processes involved in damage recovery. However, it does allow to study the early stages of damage and recovery quickly.

One advantage for using ex-situ irradiation is that large volumes of damage can be studied, which can be examined as a function of depth using cross-sectional TEM analysis. During this type of analysis the effects of irradiation can be studied, such as loop and bubble formation within metallic samples, relatively quickly.

A special case is plasma immersion whereby plasma of gas is pulsed onto the surface of the sample. This has one big advantage - the area of the sample which can be irradiated is only limited by the size of the chamber, and high fluences $\left(\mathrm{cm}^{-2}\right)$ can be undertaken quickly. One disadvantage of this technique is the energy of incoming ions tends to be much lower than ion beam irradiation from accelerators, but for shallow damage close to the sample surface, this may be an adequate solution.

There are a few ways by which the high level of damage can be described. The most common is to use displacements per atom (dpa). This unit allows comparison between ion beam, electron beam and neutron irradiation damage. The method for converting fluences to dpa for ion beam irradiation ls: $=\frac{D v}{\rho a}$, where $D$ is the number of displacement per unit length per ion, $v$ is the fluence of atoms per unit area, and $\rho_{\mathrm{a}}$ is the atomic number density. The value which is pre-calculated is $D$, the displacements per unit length per ion which can be found using damage simulation codes such as SRIM [11].

Of particular importance for oxide semiconductor surfaces is the case of creating surface defects. In Fig 5 is shown schematically the (110) structure of rutile, where the two characteristic positions for $\mathrm{O}$ (red) are visible: in-plane and out-of-plane.

Ion irradiation can more easily remove the out-of-plane (bridging) surface oxygen, forming defects. This is introducing an inter-band donor state, below the Fermi level, with a Ti-3d character, leading to the formation of $\mathrm{n}-\mathrm{TiO}_{2}$. Heat treatment in reduced oxygen partial pressure may have a similar effect.

\section{Particle Induced X-ray Emission (PIXE)}

When the probe ions collide with target atoms on or under the surface, one possible consequence is the ionisation of the target atoms, and the subsequent emission of characteristic X-rays.

PIXE is a surface and near surface analysis technique, and is suitable for analysis of target elements heavier than Al. The depth of analysis is dictated by the end of range of an incident ion which is determined by the type of the sample matrix, the energy of probe ion and the type of probe ion. The analysis is usually carried out with protons between $1 \mathrm{MeV}$ and $3 \mathrm{MeV}$, but for specific samples other probe ions such as $\mathrm{He}$ or $\mathrm{C}$ may be used. A typical PIXE spectrum obtained with $2.5 \mathrm{MeV}$ protons is shown in Figure 6 , for a thick sample of glass.

The characteristic X-rays are detected, and the quantitative analysis is achieved by a calibration procedure with known standard samples, by direct comparison of the area 
under the respective peaks. Optimum detection sensitivity is near Fe (1ppm), and other element sensitivities range from 1 - 100 ppm, which is about 100 times better than EDX, and is mainly due to a much lower background. For proton PIXE the typical depth of analysis ranges from $20 \mu \mathrm{m}$ to $100 \mu \mathrm{m}$, but the depth information is absent $[14,15]$.

PIXE can be carried out with a broad ion beam or with a focused ion beam. In the latter case, the ion beam is focused down to micron or even sub-micron size and rasterscanned over the analysed area, which confers the additional capacity to extract spatial information on the surface distribution of a particular element, with a lateral resolution of less than $0.5 \mu \mathrm{m}$. In this way, surface X-ray mapping of element distribution can be measured.

PIXE is a powerful tool in the determining the concentration of a wide range of secondary elements, which are added intentionally (doping) or unintentionally (impurities). Since the presence of aliovalent ions already at the level 5-10 ppm results in substantial changes in properties [1] the characterisation of semiconductors should include the determination of the impurities.

\section{Rutherford Backscattering (RBS)}

The technique which measures the energy of an ion-projectile scattered from target atoms in materials provides information about their composition depth profile in the near surface region. Surface degradation, such as corrosion or exposure to ultra-violet radiation can be examined by the RBS to determine occurred changes in elemental composition of tested materials.

Low energy $(<0.1 \mathrm{MeV})$ ion beams scatter from solid surfaces and, as a result, may be used to determine the composition of surfaces. Intermediate energy (0.5 - 5.0 MeV) beams of light ions, like $\mathrm{H}^{+}, \mathrm{D}^{+}, \mathrm{He}^{+}$, penetrate to a considerable distance below the surface of a material and thereby enable us to probe the composition depth profile of a sample below the surface. When high energy ions penetrate a material they lose energy in direct proportion to the thickness of material traversed, so that a depth scale can be assigned quantitatively to the energy spectrum of detected particles. The composition of a material at a particular depth is determined from the measured energy loss of backscattered ions and the scattering cross sections of the atoms present.

When energetic light ions such as $\mathrm{H}^{+}$or $\mathrm{He}^{+}$move through a solid, energy loss occurs mainly via interaction with electrons which are raised to excited energy states or ejected from the atoms. Energy loss by interaction with nuclei is small compared with interaction with the electrons and therefore, to a first approximation the nuclear interaction can be neglected.

Ion collisions with target atoms are divided into two regimes depending on the velocity of the incident ions. When the ion velocity is large compared with the velocity of an orbital electron, the collision may be regarded as a sudden small perturbation producing a sudden transfer of energy to the target electron. The energy loss from the ion to the essentially stationary electron in this high energy regime can be calculated using the theory of scattering in a central force field. Under these circumstances the stopping power (cross section) decreases with increasing ion velocity. When the ion velocity is small compared with the orbital electron velocity the scattering can no longer be treated as a central force problem and the stopping power is found to be proportional to the ion velocity. The maximum in stopping power occurs at the energy separating these two ion velocity regimes, and backscattering spectrometry is concerned with the region near and above the maximum stopping power. 
$=2.2 \times 10^{6} \mathrm{~m} \mathrm{~s}^{-1}$, which is equivalent to the velocity of a $25 \mathrm{keV} \mathrm{H}^{+}$ion. Thus, if the energy of incident hydrogen ion beam is much larger than $25 \mathrm{keV}$ it will be in the high energy regime.

The variation of ion energy with depth below the surface of a material is given by:

$$
-\frac{d E}{d x}=\frac{4 \pi Z^{2} e^{4} N Z_{2}}{m v^{2}} \operatorname{Ln}\left(\frac{2 m v^{2}}{I}\right)
$$

$Z_{1} \quad$ is the atomic number of the incident ion (projectile) $Z_{2}$ is the atomic number of the target atom $\mathrm{E} \quad$ is the charge on the incident ions, $\mathrm{N} \quad$ is the number of target atoms per unit volume, $\mathrm{M} \quad$ is mass of an electron in the target atoms, I is the average excitation energy of electrons in the target atoms, and $v$ is the velocity imparted to electrons by collision.

For $\mathrm{H}^{+}$ions of a few $\mathrm{MeV}$ energy, relativistic effects are negligible and equation 1 can be used to calculate $\mathrm{dE} / \mathrm{dx}$.

The rate of energy loss as a function of distance below the surface of the target material from the ions incident on the target nuclei can be estimated by a similar method to that used to derive equation 1 giving the following result

$$
\left.\frac{d E}{d x}\right|_{n}=\frac{4 \mathrm{Z}_{2}^{2} \mathrm{Z}_{1}^{2} \mathrm{e}^{4} \mathrm{~N}}{M_{2} v^{2}} \operatorname{Ln}\left(\frac{b_{\max }}{b_{\min }}\right)
$$

The ratio of energy loss from the incident ions to nuclei, to energy loss to electrons can be estimated by taking the ratio of equation (2) to equation (1), neglecting the logarithmic terms, and shows that the rate of loss to nuclei is about $1 / 3600$ of the rate of loss of energy to electrons.

\section{Backscattering From Thin Films}

In thin film analysis the total energy loss, $\Delta \mathrm{E}$, from ions traversing a distance t of material is, to a good approximation, proportional to $\mathrm{t}$ giving:

$$
\Delta E_{\text {in }}=\left.\int \frac{d E}{d x} d x \cong \frac{d E}{d x}\right|_{\text {in }} \cdot t
$$

where $[\mathrm{dE} / \mathrm{dx}]_{\text {in }}$ is evaluated at an average energy between the incident ion beam energy $E_{o}$ and the energy at depth $t, E_{o}-t[d E / d x]$.

After large angle scattering through angle $\theta$ the particle loses energy along the outward path and emerges with energy given by:

$$
{ }_{|\quad|} E_{1}(t)=K E(t)-\left.\frac{t}{\cos \theta} \frac{d E}{d x}\right|_{\text {out }}
$$

where the kinematic factor $\mathrm{K}$ is the ratio of the projectile energies before and after a collision, and $[\mathrm{dE} / \mathrm{dx}]$ is evaluated at the average energy of the ions as they traverse the outward path. The energy width of the spectrum expected from a film of thickness $\Delta t$ is then given by:

$$
\Delta \mathrm{E}=\Delta \mathrm{t}\left(\left.K \frac{d E}{d x}\right|_{\text {in }}+\left.\frac{1}{\cos \theta} \frac{d E}{d x}\right|_{\text {out }}\right)
$$


The backscattering spectrum at $\theta=170^{\circ}$ for $3 \mathrm{MeV}^{4} \mathrm{He}^{+}$ions incident on a $400 \mathrm{~nm}$ aluminium film with thin Au coatings consisting of about 3 monolayers of Au atoms at the bottom \& top surfaces of the aluminium is shown in Figure 7 . 
At the incident energy of $3 \mathrm{MeV}$, on the way in, the energy loss along the forward path is $220 \mathrm{eV} / \mathrm{nm}$, and after the interaction with a target atom, the energy loss on the way out is $290 \mathrm{eV} / \mathrm{nm}$ at the detected energy of about $1.5 \mathrm{MeV}$. The kinematic factor $\mathrm{K}$ for Al is approximately 0.55 . Inserting these values into equation 5 , it gives a peak width of $165 \mathrm{keV}$ for the Al peak. The energy separation between the Au peaks can be calculated similarly using $\mathrm{K}$ for Au in equation 5 together with $[\mathrm{dE} / \mathrm{dx}$ ] for Al. The Au peak separation estimated from equation 5 is $195 \mathrm{keV}$.

When the film thickness or path becomes appreciable, a better approximation to the above theory can made by selecting a constant value of $\mathrm{dE} / \mathrm{dx}$ at a mean energy, $<\mathrm{E}>$, intermediate between the values at the end points of each track. Values for the scattered peak width $\Delta \mathrm{E}$ calculated by this method differ from the result determined using equation 3.6 by approximately $3 \%$ at $2.0 \mathrm{MeV}$ ion

energies. As a result either method of analysis can be used to determine backscattered spectral line widths. The backscattered spectrum obtained in this way can be used to obtain a linear depth profile of elements within a sample.

\section{Backscattering from a Thick Sample}

The energy spectrum of backscattered $1.4 \mathrm{MeV} \mathrm{He}^{+}$ions from an infinitely thick $\mathrm{Au}$ target, for which ions come to rest inside the target, has the characteristic shape shown in Figure 3.5, which can be predicted using equation, (5) and the relationship for the energy dependence of the Rutherford scattering cross section.

In backscattering experiments the detector subtends a solid angle $\Omega$ so that the number of detected particles $Y$ scattered from a thin layer of target atoms, $\Delta t$, is given by:

$$
Y=\sigma(\theta) \Omega Q N \Delta \mathrm{t}
$$

where $Q$ is the measured number of incident particles and $N$ is the number of target atoms per unit volume in the thin layer. For thick targets, ions can scatter from a range of depths, $t$, resulting in a continuous energy spectrum to low energy. The yield from a slice of width $\Delta t$ at depth $t$ and at a scattering angle of $180^{\circ}$ is given by:

$$
Y(t)=\left(\frac{Z_{1} Z_{2} e^{2}}{4 E(t)}\right)^{2} N Q \Omega \Delta \mathrm{t}
$$

where $E(t)$ is the energy of an ion at depth t. In backscattering we obtain the spectrum of the scattered ion energies, $E_{1}$, for the ions scattered out of the target material.

If $\Delta E_{\text {in }}=E_{0}-E(t)$ is the energy loss on the inward path and $\Delta E_{\text {out }}=K E(t)-E_{1}$ is the energy loss on the outward path, the ratio $\mathrm{A}=\Delta \mathrm{E}_{\text {out }} / \Delta \mathrm{E}_{\text {in }}$ is approximately constant for slowly varying energy loss as is the case for $1.4 \mathrm{MeV} \mathrm{He}^{+}$ions. Then it is easily shown by rearranging the expression for $A$ that

$$
E(t)=\frac{E_{1}+A E_{0}}{K+A}
$$

For medium to heavy atomic mass target materials $K \approx A \approx 1, E(t) \approx\left(E_{0}+E_{1}\right) / 2$, and

$$
Y\left(E_{1}\right)=\frac{1}{\left(E+E_{1}\right)^{2}}
$$

The spectral shape corresponding to equation 9 is plotted as the dashed curve in Figure 8. 


\section{Examples of Depth Profiles using RBS}

\section{(a) Implanted substrate}

For dilute concentrations of implanted species the stopping cross section is determined by the host atoms. Figure 9 shows the spectrum of Eu implanted into $\mathrm{Si}$, at a certain depth $\mathrm{t}$. The energy scale on the horizontal axis can be converted to a depth scale using equation 5 and substituting $\mathrm{K}=\mathrm{K}(\mathrm{Eu})$ and $\mathrm{dE} / \mathrm{dx}$ for $\mathrm{Si}$. The energy variation $\Delta \mathrm{E}$ gives the distance below the surface at which the maximum concentration of Eu occurs.

\section{(b) Thin Films}

The backscattered spectrum of a $20 \mathrm{~nm}$ film of $\mathrm{SiN}_{\mathrm{x}}$ deposited on $\mathrm{Si}$, and obtained with $1.8 \mathrm{MeV} \mathrm{He}$ ions, is shown in Figure 10. Nearly all of the incident ${ }^{4} \mathrm{He}^{+}$ions penetrate microns into the target before scattering.

lons scattered from the front surface of the $\mathrm{SiN}_{\mathrm{x}}$ film have an energy given by $\mathrm{E}_{1}=\mathrm{KE}_{0}$ where the kinematic factor $\mathrm{K}$ for backscattering of ${ }^{4} \mathrm{He}^{+}$at $170^{\circ}$ is 0.31 for $\mathrm{N}$ and 0.56 for $\mathrm{Si}$. Thus, the $\mathrm{Si}$ atoms in the top layer of the $\mathrm{SiN}_{\mathrm{x}}$ film will backscatter He at an energy $\mathrm{E}_{1}=1016 \mathrm{keV}\left(\mathrm{K}_{\mathrm{si}} \mathrm{E}_{0}\right)$. When ions traverse the $\mathrm{SiN}_{\mathrm{x}}$ target film on the inward path they lose energy at a rate of $100 \mathrm{eV} \mathrm{nm}^{-1}$. Therefore a $1.8 \mathrm{MeV}$ He particle will lose about 20 $\mathrm{keV}$ of energy in penetrating the $\mathrm{SiN}_{\mathrm{x}}$ film to the $\mathrm{SiN}_{\mathrm{x}} \mathrm{Si}$ interface. Immediately after scattering from the interface, He ions backscattered from $\mathrm{Si}$ in the first layer of substrate, and will have energy of $E_{2}=996 \mathrm{keV}\left[\mathrm{K}_{\mathrm{si}}\left(\mathrm{E}_{0}-20\right)\right]$. On the outward path particles will have a slightly different energy loss of $110 \mathrm{eV} \mathrm{nm}^{-1}$, so that the particles scattered from $\mathrm{Si}$ in the $\mathrm{SiN}_{\mathrm{x}}$-Si interface (or the first layer of $\mathrm{Si}$ substrate) will emerge from the $\mathrm{SiN}_{\mathrm{x}}$ film with energy of $976 \mathrm{keV}$. The energy difference expected between ions scattered from the surface and the $\mathrm{SiN}_{\mathrm{x}}-\mathrm{Si}$ interface is about $41 \mathrm{keV}(\Delta \mathrm{E})$.

Derivation of the rate of energy loss with penetration depth $\frac{d E}{d x}$ for ions incident on a solid.

In 1913 Bohr derived an expression for the rate of energy loss of a charged particle that penetrates a solid on the basis of classical considerations. He considered a heavy particle, such as an a particle or a proton, of charge $Z_{1} e$, mass $M$, and velocity v passing a bound electron of a target atom of mass $m$ at a distance $b$, see Figure 11. As the heavy particle passes, the Coulomb force acting on the electron changes direction continuously. If the electron moves negligibly during the passage of the heavy particle, the impulse, $\int \mathrm{F} \mathrm{dt}$, parallel to the path is zero by symmetry, since for each position of the incident particle in the $-x$ direction there is a corresponding position in the $+x$ direction which makes an equal and opposite contribution to the $x$ component of the momentum. However, throughout the passage, there is a force in the y direction, and momentum $\Delta p$ is transferred to the electron. This energy transfer is similar to the Coulomb force scattering used to derive the Rutherford scattering law.

Rutherford considered the process in which a charged particle undergoes a head on collision with a nucleus and transfers all of its kinetic energy into electrostatic potential energy at the point of closest approach, b. Equating kinetic energy at large distance with electrostatic energy at position b, we have

$$
\frac{1}{2} M v^{2}=k \frac{z_{1} e q}{b}
$$

in [cgs] units $\mathrm{k}=1$. If we consider collision with an electron, $\mathrm{q}=\mathrm{e}$, and we have:

$$
M v=\Delta p=\frac{2 Z_{1} e^{2}}{v b}
$$

where $\Delta p$ is the change in momentum of the charged particle in [cgs] units, and this 
will be equal to the momentum change of the electron during collision.

If the electron has not achieved a relativistic velocity, its kinetic energy is given by

$$
\frac{(\Delta p)^{2}}{2 m}=\frac{2 Z_{1}^{2} e^{4}}{b^{2} m v^{2}}=T
$$

where $\mathrm{T}$ is the kinetic energy transfer in the collision.

The differential cross section, $d \sigma(T)$, for an energy transfer between $T$ and $T+d T$ is

$$
d \sigma(T)=-2 \pi b d b
$$

and the energy loss per unit path length, $d E / d x$, is

$$
-\frac{d E}{d x}=n \int_{T_{\min }}^{T_{\max }} T d \sigma
$$

where $\mathrm{n}$ is the number of electrons per unit volume. In terms of impact parameter $\mathrm{b}$,

$$
-\frac{d E}{d x}=n b \int_{b_{\min }}^{b_{\max }} T 2 \pi b d b
$$

which reduces to

$$
-\frac{d E}{d x}=\frac{4 \pi Z_{1}^{2} e^{4} n}{m v^{2}} \operatorname{Ln}\left(\frac{b_{\max }}{b_{\min }}\right)
$$

To choose a meaningful value for $b_{\min }$, note that if the heavy particle collided head on with the electron, the maximum velocity transferred to a stationary electron is $2 \mathrm{v}$ throughout the whole passage. The corresponding maximum kinetic energy, for a nonrelativistic $v$, is $T_{\max }=1 / 2 m(2 v)^{2}=2 m v^{2}$. If this value of $T$ max is inserted into equation $A 3.3$, the corresponding $\mathrm{b}$ min becomes

$$
b_{\min }=\frac{z_{1} e^{2}}{m v^{2}}
$$

If $b_{\max }$ is allowed to become infinite, $-\mathrm{dE} / \mathrm{dx}$ goes to infinity. The smallest energy an atomic electron can accept must be sufficient to raise it to an allowed excited state. If I is the average excitation energy of an electron, choose $\mathrm{T}_{\min }=\mathrm{I}$, which gives:

$$
b_{\max }=\frac{2 Z_{1} e^{2}}{\sqrt{2 m v^{2} I}}
$$

When equations 17 and 18 are substituted in equation 16, we obtain

$$
-\frac{d E}{d x}=\frac{2 \pi Z_{1}^{2} e^{4} n}{m v^{2}} \operatorname{Ln}\left(\frac{2 m v^{2}}{I}\right)
$$

This calculation is based on direct collisions with electrons in the solid. There is another term of comparable magnitude due to distant resonant energy transfer, which leads in its simplest form, to a total stopping power twice that shown above

Or:

$$
-\frac{d E}{d x}=\frac{4 \pi Z_{1}^{2} e^{4} n}{m v^{2}} \operatorname{Ln}\left(\frac{2 m v^{2}}{I}\right)
$$

$$
-\frac{d E}{d x}=\frac{2 \pi Z_{1}^{2} e^{4}}{E} N Z_{2}\left(\frac{M}{m}\right) \operatorname{Ln}\left(\frac{2 m v^{2}}{I}\right)
$$


where $\mathrm{E}=\mathrm{Mv}^{2} / 2$ and $\mathrm{n}=\mathrm{NZ} \mathrm{Z}_{2}$ with $\mathrm{N}$ given by the atomic density in the stopping medium, and $Z_{2}=$ number of electrons per atom.

Thus we can regard the electronic interactions as composed of two contributions:

(1) close collisions with large momentum transfers where the particle approaches within the electronic orbits

(2) distant collisions with small momentum transfers where the particle is outside the electronic orbits. The two contributions are nearly equal for particle velocities used in Rutherford backscattering.

In practice RBS, the probe ions are typically light ions, from $\mathrm{H}$ to $\mathrm{O}$. The energies range from $1 \mathrm{MeV}$ to a few MeV, typically below the Coulomb barrier for target atoms. The probe ions are directed towards the sample surface at an angle between $0^{\circ}$ and $90^{\circ}$ relative to the sample normal. When a probe ion encounters a heavier sample atom at or below the sample surface, a precise amount of kinetic energy will be exchanged, and the probe ion will be backscattered with a precise amount of kinetic energy, measured by a detector, such as a surface barrier detector. This backscattered energy, together with the initial (known) energy of the probe ion and the experiment geometry, are used to calculate the mass of the target atom and the location of the collision relative to the sample surface. For these reasons, RBS is a standard-less technique, and in particular $\mathrm{H}$-RBS and He-RBS, is a quasi-non-destructive technique for characterisation of surfaces, and near surface regions. It provides the depth distribution of elements and the distribution of impurities. This can reveal additional information on the condition of interfaces and the thickness of thin films and of implanted layers. Under ideal conditions a depth resolution of up to $0.05 \mathrm{ML}$ may be achieved, for example, for Au evaporated on cleaved Mica in UHV.

The RBS thickness of thin films differs from the optical thickness provided by the opticbased techniques such as SEM, as it measures the number of atoms per unit area of the beam and is therefore independent of the sample density. The flexibility in the choice of ions, energies and the experiment geometry offers the possibility to maximise the signal from a specific element, but the method is most suitable for analysing thin films and surfaces composed of elements heavier than the substrate or matrix, respectively. In addition, under specific circumstances, this technique offers information on multilayers and on interfaces, such as inter-diffusion.

For $\mathrm{TiO}_{2}$ implanted with elements heavier than Ti, RBS can offer non-destructive depth distribution of the implanted ion and therefore the measured samples can be further used for other characterisation work, such as photo-catalytic properties, thus reducing the necessity to infer identical properties for different samples characterised by the same techniques.

In Figure 12 we show the result of in-situ RBS obtained with $2.5 \mathrm{MeV}$ protons, on (110) $\mathrm{TiO}_{2}$ single crystal annealed in oxygen at $900^{\circ} \mathrm{C}$ and in $\mathrm{Ar}$ at $600^{\circ} \mathrm{C}$. This shows an unchanged oxygen stoichiometry for the as-grown and oxygen-treated sample. However, for the Ar-annealing case, the oxygen depletion is readily measurable, which allows to determine the oxygen stoichiometry, and the depth distribution of oxygen depletion in the sample under the surface.

Ion Channelling is a variation of RBS technique, described above. The main difference consists in the direction of primary beam of probe ions, and the sample, which in this case must display a long range order. The choice of probe ions in this case is almost exclusively $\mathrm{He}$, directed along specific crystallographic directions of the sample. For single crystals, under specific orientation conditions, the direction of probe ions may be aligned with specific crystallographic channels in the sample. In that case, He probe ion can penetrate along the channel and encounter a much smaller number of the target atoms with which to collide, and be back scattered. However, if the crystallographic channel is blocked by interstitials or distorted by the presence of defects, the channelled 
He ions will probe the channel integrity. At the core of this technique is the low angle forward scattering of ions by the atoms bordering the channel, and any disruption in the position of those atoms will change the angle of collision, which may result in back scattering rather than forward scattering. This may lead to the possibility of extracting additional information on the sample surface orientation, the interstitial atoms and defects $[12,13]$.

For example, for a $\mathrm{TiO}_{2}$ single crystal implanted with an ion heavier than $\mathrm{Ti}$, if channelling is carried out after ion implantation, it may reveal the type of implanted ion, its depth distribution as well as the damage that the implanted ion has created and the depth of the damage. In Figure 13 is shown the main crystallographic directions in $\mathrm{P} 42 / \mathrm{mnm}$ system of rutile, looking down along the

[001] direction. If the single crystal surface is represented by the (110) plane as in our previous examples, then for a channelling experiment, the He probe ions will be directed along [100] direction, and the detector normal will be along [010] direction. In this configuration the crystallographic channels parallel to these directions will be probe for distortions and interstitials.

In Figure 14 we show the results of channelling on (100) Si implanted with Ru at various equivalent concentrations, measured with $1.8 \mathrm{MeV} \mathrm{He}^{+}$. For comparison reasons, in the same figure it is also shown the He-RBS on a randomly-oriented $\mathrm{Si}$ and on a (100) oriented $\mathrm{Si}$ crystal before $\mathrm{Ru}$ implantation. The result indicate not only the $\mathrm{Ru}$ concentration and its depth distribution, but also the associated Si damage peak, and its depth distribution, noting the direct correlation between the Ru concentration and the level of damage.

The RBS results can be analyzed with a number of dedicated software available on the market, such as SIMNRA [18].

\section{Elastic Recoil Detection Analysis (ERDA)}

ERDA is another characterisation technique for the surface and near surface regions, and it differs from RBS only in its experimental geometry. In ERDA the probe ions, which are heavier than the matrix elements, are directed at the sample surface under a large angle $\left(60-85^{\circ}\right)$ from the sample normal. Thus, the heavier projectile having an adequate kinetic energy recoils the sample atoms, and their recoil energy is measured by a particle detector. The sample damage in this case can be higher than in RBS, but the beam flux is very small and a number of repeated examinations of the same area still provide indistinguishable results. The advantage is a complete depth resolution analysis for light as well as for heavy elements. In particular, when the energy detection is carried out with a time-of-flight (ToF) detector, the depth resolution can be greatly enhanced to $1-5 \mathrm{~nm}[12]$.

ERDA and RBS can be carried out in-situ or ex-situ, in conjunction with ion implantation or heat treatment procedures.

An example is hydrogen depth profiling. A direct measurement of hydrogen is difficult to perform, and in addition, due to elevated $\mathrm{H}$ mobility in matter, an in-situ / in-operando approach is preferred. Ion beams provide two techniques for direct hydrogen depth profiling: elastic recoil detection analysis (ERDA) and nuclear reaction analysis (NRA).

ERDA is based on the recoil of Hydrogen from the sample surface and sub-surface regions by a higher mass ion, accelerated to a suitable energy, in the range of $1-5 \mathrm{MeV}$. By measuring the energy of the recoil Hydrogen, with a simple surface barrier detector, a precise determination of its original depth in relation to the sample surface is possible, with a depth resolution of around $20 \mathrm{~nm}$. The schematics of ERDA measurement is shown in Figure 15 below. 
The probe ion in this case is ${ }^{4} \mathrm{He}$, accelerated to a primary energy of $1-2 \mathrm{MeV}$, and directed towards the sample surface at an angle $\alpha$, between $70^{\circ}$ and $80^{\circ}$. Hydrogen present on the surface of the sample will be recoiled by ${ }^{4} \mathrm{He}$ due to its higher mass, with a precise energy $E_{1}$, calculated from experimental conditions (angles and $E_{0}$ ).

At the surface of the sample:

$$
E_{1}=E_{0} \frac{4 M_{1} M_{2} \cos ^{2} \theta}{\left(M_{1}+M_{2}\right)^{2}}
$$

In addition to recoiled $\mathrm{H}$, some ${ }^{4} \mathrm{He}$ will also be scattered by the sample surface and to prevent it to reach the energy detector, a filter is used. The filter in this case can be a

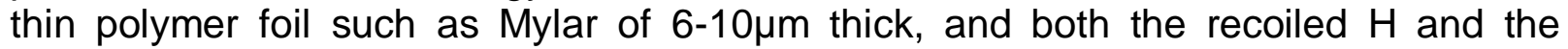
scattered $\mathrm{He}$, will lose energy traversing the foil. The precise thickness of the range foil is chosen in such a way as to stop all ${ }^{4} \mathrm{He}$, and pass all ${ }^{1} \mathrm{H}$. The energy measured by the detector in this case is:

$$
E_{d}=E_{1}-\Delta E_{\text {foil }}\left(E_{1}\right)
$$

The concentration of hydrogen $(\mathrm{N})$ is measured in [atoms $\left./ \mathrm{cm}^{2}\right]$, and it is given by

$$
N\left[\mathrm{at} / \mathrm{cm}^{2}\right]=\frac{Y[\mathrm{cts}] \cos \alpha}{N_{i} \Omega \frac{d \sigma}{d \Omega}}
$$

Where $\mathrm{Y}$ is the yield (area under the $\mathrm{H}$ peak); $\alpha$ is the angle between the beam and the sample normal; $\mathrm{N}_{\mathrm{i}}$ is the number of ions incident on sample surface; $\Omega$ is detector solid angle; $\sigma$ is the scattering cross section.

During the surface exposure to ${ }^{4} \mathrm{He}$ bombardment, some ${ }^{4} \mathrm{He}$ will penetrate the sample surface, and once inside the sample it starts to lose some of its primary energy $\left(\mathrm{E}_{01}\right)$ through interaction with sample atoms. At a depth $x$ its energy will be $E_{0 x}<E_{o}$. If ${ }^{4} \mathrm{He}$ will encounter $\mathrm{a}^{1} \mathrm{H}$ atom at that depth $x$ under the surface, it will recoil it, with the energy of the recoil $E_{1 x}$, which in this case, will be lower than the recoil energy at the surface. The recoiled $\mathrm{H}$ will lose more energy on the way to the sample surface, where its energy will be $E_{2}$. Further, the recoiled $\mathrm{H}$ will lose more energy through the filter $D E_{\text {foil, }}$ and its final detected energy will be $E_{d}$. All these energy losses can be accounted for from the experimental conditions, as follows.

At the depth $x$ under the surface:

$$
E_{1 x}=k\left[E_{0}-\frac{x}{\cos \alpha} \frac{d E_{0 x}}{d x}\right]
$$

The energy of ${ }^{1} \mathrm{H}$ leaving the sample surface will be:

$$
E_{2}=E_{1 x}+-\frac{x}{\cos \beta} \frac{d E_{1 x}}{d x}
$$

The energy measured by the detector will be: 


$$
E_{d}=E_{2}-\Delta E_{\text {foil }}\left(E_{2}\right)
$$

The result of Hydrogen depth distribution shown in Figure 16 below was obtained using the above ERDA method. The samples were polycrystalline $\mathrm{TiO}_{2}$ pressed in the form of $10 \mathrm{~mm}$ diameter pellets, and one of them was annealed at $1,000^{\circ} \mathrm{C}$ in an atmosphere of $\mathrm{Ar}+10 \% \mathrm{H}_{2}$.

ERDA can also be performed with heavier energetic projectiles, such as iodine or gold. The advantage in that case is that the heavy and energetic projectiles can recoil all the sample atoms, and thus afford the possibility for a complete sample depth composition, from the lightest $(\mathrm{H})$ to the heaviest element present in the sample. In such cases, the energy of recoiled atoms is typically measured with a time-of-flight detector, eliminating the need for a range foil, and achieving a depth resolution between $1 \mathrm{~nm}$ and $10 \mathrm{~nm}$.

In Figure 17 is shown the ERDA result using $82 \mathrm{MeV} \mathrm{I}^{+10}$ probe ions, for a LiNbO thin film deposited by CVD on (100) Si, measured with a time-of-flight detector. On the right it is shown the schematics of ERDA experimental geometry, and on the left the detection result, with each individual atoms present in the sample separated by its mass into an individual trace in the (time-of- flight vs Energy) plane. This result indicate the depth distribution of all elements present in the LiNbO film, including the $\mathrm{Li}$ isotopes, the thickness of the film ( 100ML), the presence of a $\mathrm{SiO}_{2}$ film ( 6ML thick) at the interface, and the diffusion of $\mathrm{Nb}$ in $\mathrm{Si}$ substrate.

\section{Nuclear Reaction Analysis (NRA)}

When an ion collides with the nucleus of the target atoms, if the energy is high enough to overcome the Coulomb barrier, a nuclear reaction can take place, as shown schematically in the figure below. A nuclear reaction can be written:

Ion + Target $\rightarrow$

$A+B$, or Target

(Ion, A) B

Where A, B are reaction products, and A could be another atom, a $\mathrm{Y}$-ray photon, a neutron, a proton, or a $\mathrm{He}$ nucleus. The type and energy of the reaction products is specific for each nuclear reaction, and can be used to identify the type of target atom as well as to perform quantitative analysis. This is particularly attractive for light elements, up to $\mathrm{Si}$, for which the ionization techniques are much less efficient, due mostly to two reasons: low ionization cross sections and a very steep slope of the background radiation where the characteristic peaks of light elements are present.

Depending on the nuclear reaction products, there are several types of reactions:

1- Particle-particle: an example of such reaction is ${ }^{14} \mathrm{~N}(\alpha, p){ }^{17} \mathrm{O}$, for detection of ${ }^{14} \mathrm{~N}$ using ${ }^{4} \mathrm{He}$ as the primary beam

2- Particle-Gamma reactions: an example of such reaction is ${ }^{27} \mathrm{Al}(\mathrm{p}, \mathrm{y})^{28} \mathrm{Si}$, for detection of ${ }^{27} \mathrm{Al}$ using ${ }^{1} \mathrm{H}$ as the primary beam

In the case of ${ }^{27} \mathrm{Al}(\mathrm{p}, \mathrm{y})^{28} \mathrm{Si}$ reaction the incident proton of $2.6 \mathrm{MeV}$, interact with the nucleus of target atoms $\left({ }^{27} \mathrm{Al}\right)$, and two characteristic $\mathrm{y}$-ray photons with energies of $843 \mathrm{keV}$ and $1,014 \mathrm{keV}$ are produced. The $\mathrm{y}$-rays produced can undergo Compton scattering by interacting with the electron cloud of target atoms, resulting in a continuum $\gamma$-ray background, with typical steps on the low energy side of the characteristic peaks. Some of the Compton-scattered $\mathrm{y}$-rays with energy equal or higher than $1.022 \mathrm{MeV}$ may come close to a nucleus of a target atom, and convert into electron- positron pairs, each having energy of $511 \mathrm{keV}$. Finally, the electrons and positrons produced will annihilate and two $511 \mathrm{keV}$-ray photons are produced and emitted back-to-back, for momentum 
The yield of characteristic $\mathrm{y}$-ray is proportional with the number of incident ions and target atoms, and can be used in quantitative analysis for light elements such as $\mathrm{Li}, \mathrm{F}$, $\mathrm{Na}, \mathrm{Mg}$ and Al. A typical $\mathrm{Y}$-ray spectrum from a solid sample containing $\mathrm{Li}, \mathrm{F}, \mathrm{Al}$ and $\mathrm{Na}$ is shown in Figure 18 below.

Some additional y-ray lines may be present, and could originate from the decay of naturally occurring radioisotopes present around the detector. Amongst the most abundant is ${ }^{40} \mathrm{~K}$, with a relative abundance of $0.012 \%$, and a half life of $1.28 \times 10^{9} \mathrm{y}$, whose signature is a $\mathrm{Y}$-ray line of $1,480 \mathrm{keV}$.

Another useful example for the utility of NRA is the direct measurement of hydrogen, using ${ }^{15} \mathrm{~N}(\mathrm{H}, \alpha \mathrm{y})^{12} \mathrm{C}$. This reaction has a large cross section and a narrow resonance of $1.8 \mathrm{keV}$ at a laboratory-frame energy for ${ }^{15} \mathrm{~N}$ of $6.385 \mathrm{MeV}$, resulting in a depth resolution of around $1 \mathrm{~nm}$. It is

one of the most sensitive techniques for a direct measurement of Hydrogen. It is more demanding in terms of equipment, experimental setup and procedures, but the data interpretation is relatively straightforward. The schematics of NRA is shown in Figure 19 , where ${ }^{15} \mathrm{~N}$ ions with primary energy of $6.385 \mathrm{MeV}$ are directed towards the surface to be analyzed, under a shallow angle $\alpha$, where it reacts with ${ }^{1} \mathrm{H}$ producing ${ }^{4} \mathrm{He}$ and one y quanta of $4.43 \mathrm{MeV}$. The number of ${ }^{1} \mathrm{H}$ atoms present on the surface of the sample is proportional with the number of $\mathrm{y}$ photons detected. When the primary energy of ${ }^{15} \mathrm{~N}$ is increased, the reaction resonance moves away from the surface inside the sample due to the small loss in energy of ${ }^{15} \mathrm{~N}$, which will decrease to the resonance energy of 6.385MeV at some depth $x$ under the surface, and will again react with ${ }^{1} \mathrm{H}$ if present at that depth. The previous steps are repeated until the entire depth of interest is probed. The result of Hydrogen depth distribution shown in Figure 20 below was obtained using the above NRA technique, for a thick DLC film deposited on Si.

There is a large number of nuclear reactions, and specific nuclear reactions can be used for surface and near surface characterisation, when, for example, the direct measurement of a particular element is difficult to achieve through other techniques. Light elements (up to Al) usually fall in this category, and nuclear reactions can yield prompt reaction products. In particular nuclear

reactions with narrow resonances and $y$-ray products are a first choice for depth profiling of light elements, with a depth resolution of around 10nm.

NRA can be used for both crystalline as well as for amorphous samples, and in specific cases it can be combine with the isotopic exchange kinetics, representing a powerful combination of isotope diffusion and isotope depth profile characterisation

\section{Ion Beam Induced Charge (IBIC)}

IBIC is a single-ion microprobe analytical technique [19] which is used for characterization of electronic transport properties in semiconductor microelectronic devices. It is usually carried out using focused ion beams of mostly light ions (protons and alphas). The measured signal is proportional to the charge collected by the sensing electrodes of semiconductor depletion region which is under ion bombardment. The IBIC signal depends on the free charge carrier properties, device electrostatics, surface and interface properties, and the pre-existing defects or the defects induced by the ion beam. This will be treated later in this chapter in relation to low level defects in semiconductors.

The micro beams can also be used for micro-implantation, single-ion implantation, 
micro-RBS, micro-ERDA or micro-NRA. In addition, the ionization processes induced by the micro-beam, coupled with the beam rastering on the target surface, can be used to generate elemental distribution maps using the characteristic transitions of elements present.

\section{Isotopic Exchange Kinetics}

This technique is a powerful combination of isotope diffusion and isotope depth profile characterisation, and it allows for diffusion kinetics to be determined for specific markers in various materials. It makes use of isotope-specific nuclear reactions capable to distinguish subtle variations in concentrations of specific isotopes.

For the purpose of understanding the $\mathrm{TiO}_{2}$ surface properties, partial replacement of ${ }^{16} \mathrm{O}$ with ${ }^{18} \mathrm{O}$, or the use of $\mathrm{D}_{2}{ }^{16} \mathrm{O}$, or $\mathrm{D}_{2}{ }^{18} \mathrm{O}$ instead of $\mathrm{H}_{2}{ }^{16} \mathrm{O}$ may offer advantages in monitoring surface adsorption of water, and possibly identify specific adsorption sites.

For example, the bridging oxygen in Fig 5 removed by ion bombardment or by thermal treatment may be replaced by ${ }^{18} \mathrm{O}$ instead of ${ }^{16} \mathrm{O}$, from a gas phase or from $\mathrm{H}_{2}{ }^{18} \mathrm{O}$. Surface and sub-surface ${ }^{18} \mathrm{O}$ can then be detected by elastic recoil detection (ERDA) with low energy $\mathrm{Ne}^{+}$as projectile, or by the use of specific nuclear reactions such as ${ }^{18} \mathrm{O}(\mathrm{p}, \alpha){ }^{15} \mathrm{~N}$ using proton primary beam of $845 \mathrm{keV}$.

\section{Positron Annihilation Lifetime Spectroscopy (PALS)}

The positrons emitted from a radioactive source such as ${ }^{22} \mathrm{Na}$ can penetrate the surface of a conductor or an insulator, and quickly thermalize. The decay of ${ }^{22} \mathrm{Na}$ can be described by the following:

${ }^{22} \mathrm{Na} \rightarrow \gamma^{+} \beta^{+}+v_{e}+{ }^{22} \mathrm{Ne}$

When the positron energy falls down to $511 \mathrm{keV}$ they annihilate with the free electrons existent in the material, or can undergo a spin coupling with electrons and form two quasi particles, para- positronium and orto-positronium. In all cases, the annihilation reaction is producing the characteristic back-to-back $y$-ray photons of $511 \mathrm{keV}$, but ortopositronium lifetime is approximately 140 times longer. If defects (vacancies, pores) are present in the material then the lifetime of orto-positronium will further increase, and a correlation can be established between the additional increase in the lifetime and the size of the defects.

A refinement of this technique consists of the use of a micro beam of pulsed positrons for enhanced lateral resolution which afford the possibility to measure both the lifetime and the Doppler broadening of the annihilation reaction, and produce 2D defect maps [16].

An example is taken from [16], where fluorine was implanted in $\mathrm{TiO}_{2}$ single crystal at $200 \mathrm{keV}$, and at doses between $10^{16}$ and $10^{17} / \mathrm{cm}^{2}$. After annealing in air at $1,200^{\circ} \mathrm{C}$, SIMS shows fluorine migration towards the surface, but channelling-RBS with $2 \mathrm{MeV} \mathrm{He}$ shows damage, and XPS suggest $F$ is replacing $O$. When PALS was performed with positron energies between $0.2-25 \mathrm{keV}$, it measured the broadening and $\mathrm{S}$ parameter of the annihilation response before and after implantation and recovery annealing, which indicate creation of vacancies [17].

In order to understand the effects of low level radiation damage in semiconductors, we start with a brief look at the processes which govern the interaction of ion radiation with matter. 


\section{Interaction of ions with matter}

Interaction of accelerated ions with matter is a very complex phenomenon which is the subject of research since the beginning of the 20 -th century until the present days. The main aspects of this interaction are the energy transfer from accelerated ions to the target atoms, and the modification of target composition if accelerated ions come to rest inside the target. The energy loss results in elastic and inelastic interactions with the target electrons and nuclei. A special case of inelastic interaction takes place when the accelerated ion has enough kinetic energy and overcomes the potential barrier of a target nucleus, colliding with it, and triggering a specific nuclear reaction.

The energy loss of an accelerated ion in matter is synonymous with the stopping power of the target material, measured in $\left[\mathrm{eV} \mathrm{cm}^{2}\right]$ per unit length (mono-layer, micron, etc). Figure 21 shows the stopping power of $\mathrm{Si}$ for various light ions $(\mathrm{H}, \mathrm{He}, \mathrm{Li}, \mathrm{C}, \mathrm{O}$ and $\mathrm{Cl}$ ) as a function of energy of accelerated ions indicating separately the nuclear and electronic contributions. Up to energy of approximately $100 \mathrm{MeV} /$ nucleon, the interaction is dominated by the interaction with electrons and nuclei. Above that energy, the interaction is dominated by radiative losses (bremsstrahlung, Cerenkov) and nuclear reactions. In the lower energy regime, the interaction with target nuclei results in their displacement, which can lead to point defects, whilst the interaction with target bound electrons results in ionization, and formation of cluster defects. In the case of elastic interaction, this results in scattering of ions by the target nuclei and electrons, and/or recoil of target atoms by the incident ions.

In general, an interaction of the ionizing radiation and matter is a statistical process which can be described using statistical values: (i) the cross section $\sigma$ and (ii) the mean free path $\lambda$, which are related with the following expression: $\lambda=1 / N \sigma$, where $N$ is a concentration of scattering centers in a material. The cross section determines a probabilty of interaction between a beam particle and a target particle. The angular differential cross section $d \sigma / d \Omega$ determines a probabilty for detection of interaction products in given direction, while an energy dependent differential scoss section $d \sigma / d E$ determines probability of interaction with products having an energy in the range $<E$, $E+d E>$. The mean free path is an average distance traveled by a beam particle before an interaction with a target particle. Beam particles transfer a part of their energy to target particles; their energy gradually decreases until they are brought to a rest (or transverse through material of interest carrying a final energy lower than an initial). Two mechanisms characterise an energy loss during a passage of charged particles (ions) through a solid material: (i) the energy loss due to a non-elastic scattering of a charged particle on free or atomic (binded) electrons - electronic stopping or energy loss and (ii) the energy loss due to a scattering of a screened atomic nucleus - nuclear stopping. A particle stopping can be treated as a stochastic process due to a large number of individual interactions of a beam particle and target atoms. The observed effect is a cumulative result of many events. The stopping power, $\mathrm{S}=\mathrm{dE} / \mathrm{dx}$, is an average energy loss over unit distance in a target. The stopping depends on: $a$ ) the ion velocity, b) the masses of a beam and target particle, $c$ ) the atomic number $Z$ of an ion and target atom, d) the elemental content and material density. Fig 22 shows nuclear and Fig 23 electronic stopping powers for selected ions in silicon calculated using the SRIM (Stopping and Range of lons in Material) code [20]. A scattering on atomic nuclei or nuclear stopping dominates in a case of ion velocities $\mathrm{v}$, significantly lower than the Bohr velocity $\mathrm{v} 0=\mathrm{c} / 137$. Nuclear stopping decrease with an energy increase as $1 / \mathrm{E}$, while an electronic stopping increase and eventually dominates over the nuclear stopping. In the ion velocity range $v=<0.1 \mathrm{v} 0, \mathrm{Z} 2 / 3 \mathrm{v}>$ the electronic stopping is proportional to an ion velocity, i.e. increases as $\sqrt{ } E$. For presented studies the most interesting ion velocities are in the non-relativistic range well above the Thomas-Fermi velocity, $v>>Z 12 / 3 v 0$. 
In this condition the charge state of an ion changes along its trajectory in material until it reaches its equilibrium value, and the ion electronic stopping is proportional to the square of an effective ion charge and can be expressed by the Bethe-Bloch formula:

$$
S_{e l}=\frac{4 \pi e^{2} N Z_{2} Z_{1}^{2}}{m_{e} v^{2}} L
$$

where $Z_{1}$ i $Z_{2}$ are atomic numbers of the ion and the target atom; $N$ is the atomic concentration in the target; $m_{e}$ is the electron mass; and $L=\ln \left(2 m_{e} v^{2} /<>\right)$ is the stopping number which changes slowly with ion energy. The average ionization potential $\langle>$ is an averaged ionization energy $l_{i}$ for all electronic subshells with the following satisfied condition: $2 m_{e} v^{2}>l_{i}$. It follows that the electronic stopping of the same ions is larger in a target with the higher target atomic number $Z_{2}$.

Another important parameter for studies of ion - semiconductor interactions is the end of an ion range $R$ in a material, which is the averaged distance which an ion travels in material before it loses its entire kinetic energy:

$$
R=\int_{E_{0}}^{0}(d E / d x)^{-1} d E
$$

where the stopping power $d E / d x$ includes both the electronic and nuclear stopping contributions. The end of an ion range as a function of energy for ion stopping in silicon is shown in Fig 24.

The calculated depth dependent stopping distributions for selected light and heavy ions, having an initial velocity of the order of $1 \mathrm{MeV} / \mathrm{u}$, in silicon are shown in Fig 25 (light ions) and Fig 26 (heavy ions). Presented calculated values are averaged results over 10000 incident ions. Commonly used stopping units are $\mathrm{keV} / \mathrm{ion} / \mu \mathrm{m}$ or $\mathrm{eV} / \mathrm{ion} / \mathrm{A}$. The electronic stopping of light ions like protons and alpha particles increases slowly up to a maximum value, because their velocity slowly changes with a depth in material. The proton electronic stopping dominates over almost the entire simulated energy range (Fig 23). The nuclear stopping (which doesn't contribute to the ionization) of the protons or alphas starts to dominate at velocities of approximately $0.1 \mathrm{v} 0$. Close to the end of an ion range the electron stopping deminishes, and ion stopping is entirely due to the nuclear stopping.

The electronic stopping of heavier ions in the same velocity range is substantially different from previously discussed light ions. It decreases continously at different rates as their energy decreases, and at certain energy the nuclear stopping overcomes the electronic stopping and dominates. The majority of studies utilizing accelerated ions presented here focuss on the ion energy range between $0.1 \mathrm{MeV} / \mathrm{u}$ and $1 \mathrm{MeV} / \mathrm{u}$ produced by small single ended or tandem accelerator with the highest accelerating voltage in the 1-10 MV range, and as such, the energy straggling (loss) of ions in a material can be neglected. On contrary, a spatial straggling, i.e. a lateral deviation from an initial ion direction with a depth in material, has to be taken in consideration for microprobe (raster- scanned microbeam) applications.

IBIC measurements are mostly performed using light ions, which have a significantly smaller lateral straggling than heavier ions, comparable with a microbeam resolution or a scanning resolution (pixel size). The microprobe assisted heavy ion implantation for single ion defect or radiation damage production utilizes rapidly raster scanned medium rate $(1-10 \mathrm{kHz})$ ion microbeam with a pixel dwell time of the order of 100 microseconds to generate homogenous defect distribution by intentionally or accidentally overlapping ion cascades. 
The vacancy production rate generated by ion interactions can be calculated, and in Fig. 27 this calculation is shown for a selection of light and heavy ions in silicon. A MonteCarlo simulation of the disordered region dense with primary defects following a single 8.3 MeV O ion implantation in silicon is shown in Fig. 28.

\section{Primary Defect Generation Rate Non-Ionizing Energy Loss (NIEL)}

Radiation damage correlations in semiconductors exposed to different types of ionizing radiation can be established using the Non-lonizing Energy Loss (NIEL) concept [21]. The NIEL is the rate at which energy is lost to nonionizing events (energy per unit length). It is a direct analogue of the linear energy transfer (LET) or stopping power for ionization events [22]. The NIEL concept received considerable attention recently because it was succesful to correctly decribe the observed effects of radiation damage in various simple semiconductor devices irradiated by different types of

ionizing radiation (gamma rays, heavy charged particles, and electrons) [23]. Examples have included JFET structures, solar cells, high temperature superconductors, optical sensors, and high energy ( $>\mathrm{GeV}$ ) particle detectors. The utility of the concept rests upon the fact that, to a good

approximation, displacement damage effects produced by many different particles over a wide range of energies are proportional to the nonionizing energy losses of the primary particle and the energetic nuclear recoils produced.

It is important to stress that the phenomenological NIEL concept does not take into account details about (i) the spatial distribution of primary produced defects in a particle cascade and (ii) the relaxation processes in a material after generation of a cascade leading to formation of final stable defects.

The units of NIEL are typically $\mathrm{MeV} / \mathrm{cm}$ or $\mathrm{MeV} \mathrm{cm} / \mathrm{g}$. The calculation of NIEL requires information regarding the differential cross section for atomic displacements $d \sigma / d \Omega$, the average recoil energy of the target atoms $(\mathrm{T})$, and a term which partitions the energy into ionizing and nonionizing events, called the Lindhard partition factor (L). NIEL can be written as an integral over solid angle [Sum93]:

$$
N I E L(E)=\frac{N}{A} \int_{\theta}^{\pi}\left(\frac{d \sigma(\theta, E)}{d \Omega}\right) T(\theta, E) L[T(\theta, E)] d \Omega
$$

In equation (23) $N$ is the Avogadro's number, $\mathrm{A}$ is the atomic mass, and $\theta$ is the scattering angle for which the recoil energy equals the threshold for atomic displacement. The total particle energy loss rate in a material is a sum of the NIEL and LET contributions.

The displacement damage dose $\left(D_{d}\right)$ is the total absorbed energy of an ionizing radiation for a displacement damage per unit mass of a materials, and can be expressed as a product of the average NIEL value and the radiation dose $(\Phi)$ :

$$
D_{d}=N I E L_{A V} \cdot \Phi(24)
$$

The NIEL can be calculated using the Monte-Carlo code SRIM [20], Marlowe code or CrystaITRIM (SRIM adopted for crystalline material), i.e. different software for simulation of the particle - matter interaction, which is able to calculate a depth profile of the ionization energy loss

(LET) and the depth profile of primary monovacancy generation rate per unit length in a solid material. For these calculations the SRIM uses the modified Kinchin-Pease theory. A detailed procedure of NIEL calculation using the SRIM results is given in [24]. The required input parameters for calculation are: 
- Ion type and it's initial energy $E_{0}$, in [keV];

- Threshold energy for an atom displacement from its regular lattice site $T_{d}$, in [eV];

- Composition and density of (semiconductor) material - target $\rho$, in $\left[\mathrm{g} / \mathrm{cm}^{3}\right]$;

- End of an ion range $R$, in $[\mu \mathrm{m}]$.

A graphical presentation of the NIEL profile (NIEL(z)) calculation from depth profiles for the ionization rate and the vacancy generation rate given by the SRIM is shown in Fig 29 for a case of $6.5 \mathrm{MeV} \mathrm{O}$ ion penetrating at the right angle in to a silicon target.

In Fig. 30 is shown the calculated non-ionizing energy loss depth profile NIEL(z) for $6.5 \mathrm{MeV} \mathrm{O}$ ion in silicon. Is shown together with the average NIEL value (NIELAV), which is given by:

$$
N I E L_{A V}=\frac{1}{z_{0}} \int_{0}^{z_{0}} \operatorname{NIEL}(z) d z
$$

\section{Basic Properties of Semiconductor Materials}

The semiconductor materials of interest for fabrication of electronic devices, and in particular solid state detectors/sensors/monitors of ionizing radiation for operation in various conditions include:

(i) silicon grown by various crystal growth techniques (Czochralski (CZ), Float-zone (FZ), ...),

(ii) galium arsenide ( $\mathrm{GaAs}$ ), (iii) silicon carbide (4H-SiC, $6 \mathrm{H}-\mathrm{SiC}$ and $3 \mathrm{C}-\mathrm{SiC}$ ), (iv) cadmium telluride (CdTe), (v) cadmium zinc telluride (CdZnTe), (vi) mercury zinc telluride

(HgZnTe), and diamond (CVD); (vii) titanium oxide. The basic physical properties of semiconductor materials are shown in Table 2, collected from various sources in the open literature.

The basic theory of semiconductors which is required for understanding of the operation of simple semiconductor electronic structures and devices, and will be presented here was taken from the book by S.M. Sze [26]. At the finite temperature small number of electron bonds in a crystal are broken and as a result the free electrons populate states in the conductive band $(F(E)>0)$ while the free holes populate states in the valance band $(F(E)<1)$, Fig. 31. If the concentration of free electrons in the conductive band is $n$, and of free holes in the valance band is $p$, than the intrinsic free carrier concentration $n_{i}$ at the temperature $T$ is defined as:

$$
n \cdot p=n_{i}^{2}=N_{C} \cdot N_{V} \cdot \exp \left[-\frac{E_{g}}{k \cdot T}\right]
$$

where $N_{C}$ i $N_{V}$ are effective densities of states in the conductive and valence band; $k$ is the Boltzman constant, and $E_{g}$ is the band-gap energy. The semiconductor material with the same densities of states in the valance and conductive band $\left(N_{C}=N_{V}\right)$ is an intrinsic semiconductor. The temperature dependence of the intrinsic concentration can be visualized in a case of germanium: at $T=77 \mathrm{~K} \rightarrow n_{i}<10^{8} \mathrm{~cm}^{-3}$, while at the room temperature $T=293 \mathrm{~K} \rightarrow n_{i}=2.4 \cdot 10^{13} \mathrm{~cm}^{-3}$. As a contrast, the intrinsic carrier concentration in silicon at the room temperature, due to exponential dependence on the band-gap energy, is only $n_{i}=1.5 \cdot 10^{10} \mathrm{~cm}^{-3}$, i.e. in silicon only one atom in $10^{12}$ atoms contributes one electron to the conductivity.

The concentration of free electrons in the conductive band and of free holes in the valance band can be significantly modified by doping of semiconductors with either donor or acceptor states, i.e. impurity atoms whose valance number is either +1 or -1 compared with initial constitutive atoms. 
In general, the substitution of a lattice atom with an imputiry atom is adding an energy state in the forbiden energy band-gap of a semiconductor. The amount of energy required for ionization of those impurity states below the conduction or above the valance band makes a dinstinction between shallow and deep donor or acceptor states. Shallow donors or acceptors are states with the ionization energy of the order of $\sim k_{B} T$ in respect to the lower edge of conductive or the upper edge of valance band, Fig 32 and Fig 33 respectively.

In general, donors are impurities which can be neutral or positively charged, while acceptors are impurities which can be neutral or negatively charged. The charge state of an impurity depends on the ionization energy and the temperature. At the room temperature almost all shallow donor states below the conduction band and all shallow acceptor states above the valance band in doped semiconductor are ionized. In a case of the large concentration of shallow dopants, the free electron (hole) concentartion $n$ $(p)$ in conductive (valance) band is equal to the concentration of ionized donor (acceptor) atoms $N_{D}\left(N_{A}\right)$ present in a semiconductor. Two cases are distinguished: a) the donor concentration $N_{D}$ is larger than the acceptor concentration $N_{A}(\mathrm{tj} . n>p)$ and material is $n$-type semiconductor with electrons being the majority charge carriers and b) the acceptor concentration $N_{A}$ is larger than the donor concentration $N_{D}(\operatorname{tj} p>n$ ) and material is $p$-type semiconductor with holes being the majority charge carriers.

\section{BASIC PROPERTIES OF $p-n$ JUNCTION}

It is often the case that $p-n$ junctions form in semiconductors due to doping. The $p-n$ junction forms in a case of the changing dopant concentration from the excess acceptor concentration $N_{A, p}$ on $p$-type side to the excess donor concentration $N_{D, n}$ on $n$-type side of a semiconductor (Fig 34a). A gradient of the hole and electron concentration on both sides of the junction causes a difussion of majority charge carriers towards the junction and their recombination at the junction. Gradually the concentration of majority carriers on both sides of the junction decreases, giving rise to the fixed charged distribution (Fig 34b). Also, the Fermi energy in n-type material decreases and the Fermi energy in $p$ type material increases. The stationary ionized acceptor and donor atoms left on opposite sides of $p-n$ junction give rise to the potential gradient across junction $V(x)($ Fig 34c).

The potential gradient generates an electric field across the depletion region opposing further diffusion of free majority carriers. The diffusion ends when Fermi energies in both $p$ - and $n$-type semiconductors become equal. The potential difference across the depletion region can be calculated using the Poisson equation:

$$
-\frac{d^{2} V}{d x^{2}}=\frac{\rho(x)}{\varepsilon \varepsilon_{0}}
$$

In the „abrupt“ junction approximation, assuming all dopant atoms being ionized in the depletion region, the charge density can be expressed as:

$$
\rho(x)=\left\{\begin{array}{c}
e_{0} N_{D, n}\left(-x_{n}<x<0\right) \\
-e_{0} N_{A, n}\left(0<x<x_{p}\right) \\
0
\end{array}\right.
$$

where $x_{n}$ i $x_{p}$ present an extent of the depletion region in $n$ and $p$-type semiconductor respectively (Fig. 35b). 
From the condition that the final system is electrically neutral:

$$
N_{A, p} x_{p}=N_{D, n} x_{n}
$$

It follows that the extent of depletion region on both sides of the junction is inversly proportional to the doping concentration. Doping concentrations are often substantially different across each $p$ - $n$ junction in electronic devices, e.g. a highly doped $p$-type material $\left(p^{+}\right)$is brought in contact with a low doped $n$-type material $(n)$. In that case $\left(x_{p}\right.$ $<x_{n}$ ), the entire width of depletion region $\omega$ might be approximated with the extent of depletion region in the low doped $n$-type material $x_{n}$ :

$$
\omega=x_{p^{+}}+x_{n} \approx \sqrt{\frac{\varepsilon \varepsilon_{0} V_{b i}}{e_{0} N_{D, n}}}
$$

The contact potential Vbi represents the potential difference arising from formation of the p-n junction (Fig 34c). Let us assume doping concentrations across $p+-n$ junction in silicon are $p_{+} \sim 1018 \mathrm{~cm}-3$ and $n \sim 1012 \mathrm{~cm}-3$. At room temperature with the contact potential of $\mathrm{Vbi} \sim 1 \mathrm{~V}$, the width of depletion region is a few micrometers.

\section{Reversly Biased $p-n$ Junction}

In a case of the simple semiconductor elements, having various geometrical configurations, the penetration depth of an ionizing radiation is usually larger than the width of a depletion region due to formation of the contact potential barrier across the $p$ $n$ junction. The electron and hole pairs are being mostly generated in a bulk semiconductor area with no electric field present. In order to prevent the electron-hole recombination a bias $V$ of the same polarity as the built in potential $V_{b i}$ is applied to the $p-n$ junction ( $V_{b i}$ in expression (30) is substituted with $V_{b i}+V$ ). The applied bias $V$ increases the width of depletion region in a direction of the lower doped bulk material, and decreases the minority carrier current through the $p-n$ junction. The reverse bias generates the electric field which separates generated free carriers along an ionization track. In a case of semiconductor diodes used for detection of an ionizing radiation, an applied reverse bias is significantly larger than the built-in voltage $\left(V>>V_{b i}\right)$, so the width (30) as a function of bias is approximatelly:

$$
\omega(V)=\sqrt{\frac{2 \varepsilon \varepsilon_{0} V}{e_{0} N_{D, n}}}
$$

In another case, if a bias voltage of opposite polarity to the built-in voltage is applied, both the potential difference across $p-n$ junction and the width of depletion region decrease, while the minority current carrier increases, until finally the blocking barrier of $p-n$ junction for majority carriers diminishes. A current through the junction increases linear with further increase of a forward applied voltage, and the junction becomes an Ohmic conductor.

It follows that for detection of an ionizing radiation, the value of applied reverse bias has to be at least sufficient to the spread the depletion region across the whole active volume of detector (corresponding to the thickness of a low doped material). In that case, the whole charge generated by an ionizing radiation will be collected. The total collected charge determines the amplitude of a measured electronic signal corresponding to the energy of an ionizing radiation. Such bias value is called the full depletion bias $\left(V_{F D}\right)$, and for a diode of thicknes $\mathrm{D}$, it can be calculated using:

$$
V_{F D}=\frac{e_{0} N_{D, n} D^{2}}{2 \varepsilon \varepsilon_{0}}
$$

It is obvious that lower doped material $\left(N_{D, n}\right)$ of the same thickness (D) used for formation of the 
$p^{+}-n$ diode requires the lower reverse bias voltage.

The radiation damage of semiconductor in the form of defects produced by ionizing radiation might change doping in bulk semiconductor material, which leads to changes of the leakage current of minority carriers and the full depletion bias. In extreme cases the type of semiconductor might change also. Negative effects on semiconductor properties and device performance will be discussed in more detail later.

\section{Capacitance of $p$-n Junctions}

The capacitance of the depletion region created by $p-n$ junction $C\left(V_{0}\right)$ biased at a reverse bias voltage $V_{0}$ is defined as the change of a fixed charge in the depletion region for a given change of bias value:

$$
C\left(V_{0}\right)=\left.\frac{d Q}{d V}\right|_{V=V_{0}}
$$

The amount of fixed charge in depletion region of the planar $p^{+}-n$ diode is the product of the unit charge, the donor concentration and the volume of depletion region:

$$
Q(V)=e_{0} N_{D, n} A \omega(V)
$$

Substituting (34) in (33) it follows that the capacitance of a planar semiconductor diode $C(V)$ is equal to the capacity of a planar capacitor whose electrode separation is $\omega(V)$, its cross section is $A$ and relative permeability is $\varepsilon$ :

$$
C(V)=\frac{\varepsilon \varepsilon_{0} A}{\omega(V)}=\left\{\begin{array}{l}
\sqrt{\frac{\varepsilon \varepsilon_{0} e_{0} N_{D, n}}{2 V}} A, V<V_{F D} \\
\sqrt{\frac{\varepsilon \varepsilon_{0} e_{0} N_{D, n}}{2 V_{F D}}} A, V>V_{F D}
\end{array}\right.
$$

In a case of under-depleted planar semiconductor diode, the capacity decreases with bias for bias values lower than the full depletion bias, $C(V) \sim 1 / \mathrm{V}$. Ionizing radiation increases the number of defects in semiconductors, so in $n$-type silicon the effective donor concentration and consequently the capacity of a semiconductor diode increases. The lowest capacity is achieved for the full depletion bias voltage apllied to a planar semiconductor diode.

\section{Reverse Current}

The current flowing through a $p-n$ junction connected to a reverse voltage supply is called the reverse current or "dark" or "leakage" current, $I_{r}$. The reverse current of an „ideal“ or pristine semiconductor diode is the diffusion current originating from impurities, contaminations and defects, present in a bulk semiconductor or on interfaces, generated by a manufacturing process of semiconductor wafers, doping by ion implantation and subsequent annealing, or metalic contact deposition. Even the best manufacturing practices are unable to produce comercially available silicon junctions with reverse current densities below $1 \mathrm{nA} / \mathrm{cm}^{2}$.

The reverse current of a partially damaged semiconductor diode by ionizing radiation consits of two components:

- Previously discussed diffusion current Is component which saturates at high reverse biases;

- Generation current component which originates from: 1) the electron-hole pair creation from deep defect states (produced by ionizing radiation) laying close to 
the middle of energy band gap - bulk generation current ( $\left.I_{\text {bulk }}\right)$ and 2 ) the surface generation current $I_{\text {surf }}$ due to trapped

free charge carriers at interfaces.

In a respect of understanding the detrimental effects of radiation damage on the reverse current in semiconductor diodes, the bulk generation current is the most sensitive to accumulation of radiation defects. Therefore, results and a discussion about changes of the reverse current in this chapter is limited to changes in the bulk generation current due to deep defect production. In this case, only defects produced in a depletion region contribute to the reverse current. It's dependece on bias, for bias voltages below the full depletion bias value $\left(V<V_{F D}\right)$, is similar to a dependence of the width of a depletion region $\omega$ on bias:

$$
\text { Ibulk } \propto \omega \propto \sqrt{V}
$$

For reverse voltages above the full depletion bias value, this bulk generation component of reverse current will saturate. Moreover, the bulk generation current is proportional to the ionization probability of generation centers which increases with a temperature. The ionization probabilty might be related to the characteristic generation time $\tau_{g}$ :

$$
\text { Ibulk } \propto \frac{\omega}{\tau_{g}}(37)
$$

All measurements in this study were performed at room temperature, and it is worthwhile to note that lowering the temperature it reduces the value of current generated through a semiconductor due to reduced probability of ionization of active centers.

\section{Defects in Semiconductors}

Defects in semiconductors play a significant role in defining, modifying or fine tuning elecrtonic properties of a material (including some of those specified in Table 2) which are crucial for design, manufacturing, operation and performance of the semconductor devices. Defects can be classified in several different ways, and the most common classifications are according to:

$>$ Origin (intrinsic and radiation induced)

$>$ Complexity (point and cluster or extended);

$>$ Electronic properties (donors and acceptors; deep and shallow)

$>$ Electrical activity (neutral and active)

In the following we will cover all types of defects, although the emphasis of this whole book chapter is given to (i) characterization of deep defects formed after exposure of a material to irradiation by particles and photons, and (ii) changes of electronic properties of a material due to a presence of the low concentration of deep defects in the depletion region of semiconductor.

The deep level defects that act as charge carrier traps have high importance in semiconductor industry and applications of semiconductor devices [27], [28]. These defects are being created during: a) semiconductor growth process, b) electronic device fabrication and c) operation in harsh environments. We will focus our attention on defects created in semiconductor devices exposed to irradiation by ions with energies in the MeV range, although other particles like electrons and neutrons, and photons will be compared and discussed.

It is well known that high energy ionizing particles traversing through or being stopped in a sensitive volume of semiconductors, deposit part of their initial energy in atomic elastic collisions displacing atoms from their lattice sites [29]. Those primary defects might annihilate or reorganize themselves with impurities to form stable deep defects. 
Defect accumulation in reasonably low concentrations (well below an extended defect formation threshold value) might modify electronic transport properties of charge carriers introduced into active region of a device, and consequently alter or deteriorate its performance [30, 31, 32, 33, 34, 35, 36, 37, 38, 39, 40, 41]. Protons, alpha particles and other heavier ions, as well as other hadrons like neutrons and high energy leptons like electrons and mions cause radiation damage in semiconductor materials by knocking lattice atoms out of their regular sites - primary knock-out atom (PKA). As the result of such collisions above the threshold energy for atom displacement, an interstitial atom and a vacancy in a crystal lattice - Frenkel pair (Fig 35) is produced. Radiation damage production does not end just by knocking out lattice atoms from their sites by incoming projectiles. If the recoil energy is much larger than the threshold displacement energy, recoiling atoms can further displace other lattice atoms from their sites and create secondary cascades. The maximal energy $E_{R, \max }$ a particle with the mass $m_{p}$ and energy $E_{p}$ can transfer to an atom with the mass Mtarget in an elastic collision in the nonrelativistic limit is:

$$
E_{R, \max }=4 E_{P} \frac{m_{P} m_{S i}}{\left(m_{P}+m_{S i}\right)^{2}}
$$

Substituting well established values for the threshold displacement energy $E_{c} \approx 21 \mathrm{eV}$ and the threshold energy for a cluster defect formation in silicon $\approx 5 \mathrm{keV}$ [42] as ( $E_{R}=$ $\left.E_{d}\right)$ in to above equation, follows that a proton of at least $\approx 158 \mathrm{eV}$ is required for the Frenkel pair production and a proton energy of $\approx 37.5 \mathrm{keV}$ is required for a secondary cascade generation and cluster defects formation. Heavier ions than protons require less energy for secondary cascade formation $\left(\approx 11.5 \mathrm{keV}\right.$ for $\alpha$ particles, $\approx 8 \mathrm{keV}$ for ${ }^{7} \mathrm{Li}$, $\approx 5.4 \mathrm{keV}$ for ${ }^{16} \mathrm{O} \mathrm{i} \approx 5.1 \mathrm{keV}$ for ${ }^{35} \mathrm{Cl}$ ions). Therefore, heavier ions are more prolific for cascade damage and high defect concentration production compared with protons or other lighter particles (Fig 36).

Primary defects are mobile at finite temperature (in silicon above $150 \mathrm{~K}$ ), so vacancies and interstitial can immediately recombine. Simulations show that approximately $60 \%$ of Frenkel pairs recombine in silicon at the room temperature [43]. Mobile primary defects can interact with other impurity atoms or defects and form stable point defects or even small clusters of point defects.

\section{Point Defects}

Point defects are formed when an initially produced interstitial atom or vacancy combine with other existing primary defect or impurity atom. Point defects can be clasified accoring to their electrical properties (Fig 37):

\section{- Acceptors, donors, and amophoteric defects:}

Point defects with their energy levels $E_{t}$ in the forbiden energy band-gap can capture and emit electrons and holes; therefore those statets can be called traps. The trap ionization energy $\Delta E_{t}$ required for emission of an electron (hole) from a trap to the conductive (valance) band is equal to

the energy difference between the edge of the conductive $E_{C}$ (vallance $E_{V}$ ) band and the energy state of a trap $E_{t}$. Acceptors are negatively charged defects when occupied by an electron, while donors are neutrally charged defects when occupied by an electron. Amphoteric defects are those that have both the acceptor and donor state. In thermal equilibrium the defect charge state is determined by the positon of the Fermi level. If the Fermi level is above the energy of a defect, an acceptor is negatively charged $(-)$, and a donor is neutral $(0)$; if the Fermi level is below the energy of a defect, an acceptors is neutral (0), and a donor is positively charged (+). The lower charge state corresponds to an ionized defect state, and the upper to a defect state 
filled with an electron (Fig.

38). Some defects can have more than a single energy state in the band-gap. Examples are thermal double donors (TDD) and amphoteric divacancies (VV). The defect population in the depletion region of a semiconductor depends on defect emission coeficients (probabilities); defects in the upper half of the bandgap are usually unoccupied, while defects in the lower half are usually occupied by electrons. It follows that in silicon for example, the vacancy - oxygen complex $\mathrm{VO}_{\mathrm{i}}$ (acceptor in the upper half of the band-gap) and the carbon - oxygen complex $\mathrm{C}_{i} \mathrm{O}_{i}$ (donor in lower half) are neutral in depletion region; so they don't have an influence on the full depletion reverse bias of partly damaged silicon.

\section{- Shallow and deep defects (traps):}

The charge state in thermal equilibrium is determined by the Fermi level, and therefore depends of the type of a semiconductor and the doping concentration. Therefore, a commonly used approach that shallow acceptors and donors are defects ionized at the room temperature can be missleading. It is more appropriate to state that a defect is shallow in a case the ionization energy is less than $70 \mathrm{meV}$.

\section{- Electron vs hole traps}

The clasification of defects on electron and hole traps, which follows from characteriazation techniques of electrical properties of defects based on changes to a distribution of space charge in depletion region of semiconductor connected to a modular source of external bias voltage (DLTS, TSC,..) is also missleading, because any defect can capture both an electron and a hole. A defect in upper half of the band gap below the Fermi level in $\mathrm{n}$-type semiconductor is occupied with electron in thermal equilibrium. The applied reverse bias extends the depletion region, and captured electrons are being emitted for these states. Measurement of the electron emission by changes to the junction capacitance as a function of the temperature enables determination of the defect ionization (activation) energy and the capture cross section of a defect, but it is not possible to conclude whether a defect which emitted an electron is an acceptor or a donor. Therefore defects characterised by junction capacitance techniques are called the electron or the hole trap. Detailed information about defects, their charge states, activation energies and annealing temperatures for silicon are given later.

\section{Cluster Defects}

The cluster defect model was first introduced by B.R. Gossick [44] to explain a high recombination rate of minority charge carriers in silicon irradiated by heavy particles compared to a low rate in silicon irradaited by electrons and gamma radiation. Despite early pioneering work by Gossick,

only few studies dealing with cluster defects have been published since then, limiting the present knowledge to evidences that cluster defects consist of larger number of vacancies or interstitial atoms. Simulation of the radiation damage created in semiconductors due to prolonged exposure in very intense radiation enivoroments (large hadron colliders, outer space, fussion reactors) supported by availability of super computers capable of handling enormous quantity of data reignited recent activities in this field of research. Monte-Carlo (MC) models based on PKA approximation and molecular dynamics models have been used for simulation of defect production by individual projectiles in a variety of materials, especially ions.

The following discussion is limited to a low level radiation damage which does not lead to substantial changes of electronic properties like a doping type or a carrier drift velocity in the depletion region, or even total destruction by extended material structural changes of studied devices. 
Trap Occupancy in Thermal Equilibrium and Concentration of Free Charge Carriers

The probability for the electron occupancy of the energy state $E$ is defined by Fermi-

Dirac distribution:

$$
F(E)=\frac{1}{1+\exp \left(\frac{E-E_{F}}{k T}\right)}
$$

where $k$ is the Boltzman constant, $T$ is the temperature in $[K]$ and $E_{F}$ is the energy of the Fermi level. The Fermi level corresponds to an energy value for which the probability for the electron occupancy is equal to one half. The concentration of free charge carriers ( $n$ - electrons and $p$ - holes) in the conductive or valance band can be calculated from following equations using the known Fermi energy:

$$
\begin{aligned}
& n=\int_{E_{C}}^{E} d E g_{C}(E) \frac{1}{1+\exp \left(\frac{E-E_{F}}{k T}\right)} \\
& p=\int_{E_{C}}^{E} d E g_{V}(E) \frac{1}{1+\exp \left(\frac{E_{F}-E}{k T}\right)}
\end{aligned}
$$

where $E g c, v(E)$ is the density function of states in the conductive $(C)$ and the valance $(V)$ band, and $E_{C}$ and $E_{V}$ are energies of band edges. The formulae (40) and (41) are valid for all semiconductors, including those which contain impurities and defects that modifiy free carrier densities and the Fermi energy level compared to the intrinsic semiconductors. Commonly assuming that $\left|E_{C, V}-E_{F}\right|$ $>3 k T$, the above formulae simplifies to:

$$
n, p=N_{C, V} \exp \left( \pm \frac{E_{F}-E_{C, V}}{k T}\right)
$$

The effective densities of states in the conductive $\left(N_{C}\right)$ and valance $\left(N_{v}\right)$ band can be calculated by following expression:

$$
N_{C, V}=2\left(\frac{2 \pi m_{d C, d V}^{*} k T}{h^{2}}\right)^{3 / 2}
$$

Where $m_{d c}^{*}$ and $m_{d v}^{*}$ are effective masses of states in the bands. Earlier, temperature independent values for silicon $1.084 \mathrm{mo}_{0}$ and $0.549 \mathrm{mo}$ from the most popular textbook of semiconductor theory [26], should be replaced by new temperature dependent values which are $1.091 \mathrm{mo}_{0}$ and $1.153 \mathrm{mo}_{0}$ at the room temperature (300K) [45]. Details about calculation of effective masses can be found in work by M.A. Green et al.

\section{Fermi Energy}

In a case of the intrinsic semiconductor the free electron and hole concentrations are 
equal $(n=p)$, the intrinsic Fermi energy $E_{i}$ is given by:

$$
E_{i}=\frac{E_{C}+E_{V}}{2}+k T \operatorname{Ln}\left(\frac{N_{V}}{N_{C}}\right)=\frac{E_{C}+E_{V}}{2}+\frac{3}{4} k T \operatorname{Ln}\left(\frac{m_{d C}^{*}}{m_{d V}^{*}}\right)
$$

and intrinsic carrier concentration is:

$$
n_{i}=\sqrt{N_{C} N_{V}} \exp \left(-\frac{E_{g}}{k T}\right)
$$

$E_{g}=E_{C}-E_{V}$ is the width of the energy band gap. Using (44) and (45) the expresion for free carrier concentrations (42) becomes:

$$
n, p=n_{i} \exp \left( \pm \frac{E_{F}-E_{i}}{k T}\right)
$$

Note that $n^{2}=n p$ even for the extrinsic semiconductor in thermal equilibrium.

If the total concentration of defects (traps) is $N_{t}$ and the trap energy level in the energy band gap is $E_{t}$, the trap concentration filled by electrons $n_{t}$ and holes $p_{t}$ can be calculated for the known Fermi energy:

$$
\begin{gathered}
n_{t}=N_{t} \frac{1}{1+\exp \left(+\frac{E_{t}-E_{F}}{k T}\right)}=N_{t} F\left(E_{t}\right) \\
p_{t}=N_{t} \frac{1}{1+\exp \left(-\frac{E_{t}-E_{F}}{k T}\right)}=N_{t}\left(1-F\left(E_{t}\right)\right)
\end{gathered}
$$

Assuming that $N_{t}=n_{t}+p_{t}$, i.e. the trap which is not occupied by an electron is occupied by a hole, and vice versa, the trap which is not occupied by a hole is occupied by an electron.

Similarly, an acceptor occupied by an electron is negatively charged, while a donor occupied by an electron is neutral, and vice versa, an acceptor occupied by a hole is neutral, while a donor occupied by a hole is positively charged. It follows that the effective concentration of trapped charge carriers $N_{\text {eff }}$ is:

$$
N_{\text {eff }}=\sum_{\text {donors }} p_{t}-\sum_{\text {acceptors }} n_{t}
$$

In the thermal equilibrium the total charge, taking into an account the free carrier concentrations $p$ i

$n$ has to be zero, i.e.

$$
0=p-n+N_{e f f}
$$

The relations (42), (43) and (47-50) determine the Fermi energy of an extrinsic semiconductor at any given temperature.

\section{Gibbs Free Energy}

Changing the charge carriers between the defects and the conduction band and valence band is treated as a statistical process. According to thermodynamic theory instead of energy we use Gibbs free energy $\mathrm{E}$, so that the change of energy becomes the change 
in Gibbs free energy when the emission of charge carriers from the traps: $A E=D H$ $\mathrm{T} \Delta \mathrm{S}$, where the $\mathrm{DH}$ is the change in enthalpy and Ds the change in entropy. If we introduce the entropic factors $X_{n, p}$ defined by the $X_{n, p}=\exp \left(\Delta S_{n, p} / k T\right)$ for change in energy during the emission of electrons and holes from the traps we get the following expressions:

$$
\begin{aligned}
& E_{C}-E_{t}=\Delta H_{n}-k T \operatorname{Ln}\left(X_{n}\right) \\
& E_{t}-E_{V}=\Delta H_{p}-k T \operatorname{Ln}\left(X_{p}\right)
\end{aligned}
$$

These terms can be used to calculate emission coefficients from the coefficients of traps.

\section{SHOCKLEY-READ-HALL Statistics}

The occupancy of traps in the energy ban gap by charge carriers is determined by the interaction of traps and charge carriers in the bands. According to the Shockley-ReadHall statistics [Sho52][Hal52] four different interaction processes are distinguished (Fig 39):

a) electron emission into the conducting band;

b) electron capture by a non-occupied trap;

c) hole capture from the valance band by a trap occupied by an electron (i.e. electron emission from a trap into valance band);

d) hole emission from a trap into the valance band (i.e. electron capture from the valance band).

Those four processes are described by emission (capture) rates $r_{i}$. The electron (hole) emission rate to the conductive (valance) band $r_{a}\left(r_{d}\right)$ are proportional to the ratio of traps occupied by electrons $n_{t}$ (holes $p_{t}$ ):

$$
r_{a}=e_{n} n_{t}, r_{d}=e_{p} p_{t}
$$

where $e_{n}\left(e_{p}\right)$ is the proportionality factor corresponding to the probabilty of an electron (hole) emission per unit time.

The electron (hole) capture rate from the conductive (valance) band by a trap $r_{b}\left(r_{c}\right)$ are proportional to the ratio of traps occupied by holes $p_{t}$ (electrons $\left.n_{t}\right)$ and the free electron $n$ (hole $p$ ) concentration in the conductive (valance) band:

$$
r_{b}=c_{n} n p_{t} \text { and } r_{c}=c_{p}
$$

where the proportionality factor $c_{n}\left(c_{p}\right)$ corespond to the probabilty of free electron (hole) capture by a trap. It follows the rate of trap occupancy $d n_{t} / d t$ is given by

$$
\frac{d n_{t}}{d t}=-r_{a}+r_{b}-r_{c}+r_{d}=-e_{n} n_{t}+c_{n} n p_{t}-c_{p} p n_{t}+e_{p} p_{t}
$$

Moreover, the concentration of free electrons in conducting band and the concentration of free holes in valance band are constant according to the principle of detailed charge balance. Therefore, the carrier interaction within the conduction band (processes «a» and $\langle b »)$, and the carrier interaction with the valance band (processes «c» and «d») 
have to proceed at the same rates. Using the principle of detailed balance and equations for concentrations of the free and trapped charge carriers

(50) the following relationship between the emission coefficients $e_{n, p}$ and capture coefficients $c_{n, p}$ is obtained:

$$
\begin{gathered}
e_{n, p}=c_{n, p} n_{j} \exp \left( \pm \frac{E_{t}-E_{i}}{k T}\right)=c_{n, p} N_{C, V} \exp \left( \pm \frac{E_{t}-E_{C, V}}{k T}\right) \\
=c_{n, p} N_{C, V} X_{n, p} \exp \left(-\frac{\Delta H}{k T}\right)
\end{gathered}
$$

Additionally, it is useful to relate the entropy factor $X_{n, p}$ and the capture coefficient $c_{n, p}$ using the cross section for a carrier capture $\sigma_{n, p}$ :

$$
c_{n, p} X_{n, p}=\sigma_{n, p} v_{t h, n, p}
$$

and the thermal electron (hole) velocity $v_{t h, n, p}$ :

$$
v_{t h, n, p}=\sqrt{\frac{3 k T}{m_{d C, V}^{*}}}
$$

\section{Equations of Free Charge Carrier Motion}

The motion of charge carriers in a semiconductors are defined by the following relations:

- Poisson equation:

$$
\frac{\partial E}{\partial x}=\frac{q_{0}}{\varepsilon \varepsilon_{0}}\left(n+p+N_{e f f}\right)
$$

- Continuity equations:

$$
\frac{\partial n}{\partial \mathrm{t}}=G_{\text {ext }}+\sum_{\text {traps }} e_{n} n_{t}-n \sum_{\text {traps }} c_{n} p_{t}+\frac{1}{q_{0}} \frac{\partial j_{n}}{\partial \mathrm{x}}
$$

and

$$
\frac{\partial p}{\partial \mathrm{t}}=G_{\text {ext }}+\sum_{\text {traps }} e_{p} p_{t}-p \sum_{\text {traps }} c_{p} n_{t}+\frac{1}{q_{0}} \frac{\partial j_{p}}{\partial \mathrm{x}}
$$

where $j_{n}$ and $j_{p}$ are the electon and hole current densities, and $G_{e x t}$ is the generation current of electron - hole pairs (e.g. ionization by ions or photons);

- Carrier current density equations:

$$
j_{n}=q_{0} \mu_{n} n E+q_{0} D_{n} \frac{\partial \mathrm{n}}{\partial \mathrm{x}}
$$

The total carrier current density consists of: (i) the drift component due to an electric field $E$ and the diffusion component due to a gradient of carrier concentration $(\partial n / \partial x$, $\partial p / \partial x) . \mu_{n}$ and $\mu_{p}$ are the electron and hole mobilities, and $D_{n, p}$ the carrier diffusivity related to mobilities through the Einstein relation $D_{n, p}=\left(k T / q_{0}\right) \mu_{n, p}$.

The total current density is the sum of individual carrier current densities $\left(j_{n}+j_{p}\right)$ and the displacement current due the electric field strength change $\partial E / \partial t$ : 


$$
j_{\text {total }}=j_{n}+j_{p}+\varepsilon \varepsilon_{0} \frac{\partial E}{\partial t}
$$

with the following condition satisfied:

$$
\frac{\partial j_{\text {total }}}{\partial x}=0
$$

\section{Trap Occupancy in the Depletion Region of Semiconductors}

The $p-n$ junction, and the corresponding depletion region forms when $p$ - and $n$-type semiconductor are brought into contact. The depletion region widens if a reverse bias is applied to the $p-n$ junction, as mentioned above. In a such condition, the equilibrium trap occupancy with charge carriers (47,

48 ) is no longer valid becuase the concentration of free charge carriers in the depletion region is negligeble $(n \approx p \approx 0)$. Traps no longer capture charge carriers and (55) becomes:

$$
\frac{d n_{t}}{d t}=-e_{n} n_{t}+e_{p} p_{t}
$$

In a stationary condition, the solution for the trap concentration filled by electrons $n_{t}$ and holes $p_{t}$ is given either by:

$$
n_{t}=N_{t} \frac{e_{p}}{e_{n}+e_{p}}, p_{t}=N_{t} \frac{e_{n}}{e_{n}+e_{p}}
$$

or, using (56) by:

$$
n_{t}, p_{t}=\frac{N_{t}}{1+\left(\frac{c_{n}}{c_{p}}\right) \exp \left( \pm 2 \frac{E_{t}-E_{i}}{k T}\right)}
$$

The effective dopping concentration $N_{\text {eff }}$ is a sum of the occupied donor (hole) and the occupied acceptor (electron) concentrations in the depletion region (the space charge region):

$$
N_{\text {eff }}=\sum_{\text {donors }} p_{t}+\sum_{\text {acceptors }} n_{t}(70)
$$

Moreover, the generation of free electron - hole pairs by a trap in the depletion region is given by:

$$
G_{t}=e_{n} n_{t}=e_{p} p_{t}=N_{t} n_{i} \frac{c_{n} c_{p}}{c_{n} \exp \left(\frac{E_{t}-E_{i}}{k T}\right)+c_{p} \exp \left(-\frac{E_{t}-E_{i}}{k T}\right)}
$$

It follows that only traps with energy states $E_{t}$ in a vicinity of the intrinsic Fermi energy $E_{i}$ contribute to the generation/recombination of carrier pairs. Experimental techniques like Deep Level Transient Spectroscopy (DLTS) are limited to characterisation of the single carrier traps and determination of the single carrier capture coefficient (either electron or hole; $c_{n}=c_{p}$ or vice versa). In this case relation (71) simplifies to:

$$
G_{t}=\frac{N_{t} n_{i} c_{n}}{2 \cosh \left(\frac{E_{t}-E_{i}}{k T}\right)}
$$


The total bulk generation current density jbulk (which determines an increase of the reverse current in partly damaged semiconductors) is a sum over all possible defect states:

\section{Crystal Lattice Defects}

The generation of primary defects in semiconductor crystal lattice, i.e. interstitial atom and vacancies is described further. Those primary defects which are mobile at a finite temperature interact with lattice atoms, impurities and other defects already present, to form final defects. Those final stable defects are being characterised by capacitive and current transient techniques.

\section{Vacancy Related Defects}

From the density functional theory (DFT) modelling it is known that the monovacancy defect $(\mathrm{V})$ in silicon, for example, can be in one of five different charge states $(--1-10 /+/+$ $+)$. Their thermal activation energies specified in the Table 3 have been taken from the book "Deep centres in semiconductors" by G. Watkins [46]. Although an existance of the acceptor states $\mathrm{V}^{(--)}$and $\mathrm{V}^{(-/ 0)}$ has been indirectly proven by the electron paramagnetic resonce, their energy levels specified in the Table 3 are results of theoretical calculations. The energy of the double donor state $\mathrm{V}^{(++/+)}$is $+0.13 \mathrm{eV}$ below the energy of the single donor state $\mathrm{V}^{(+/ 0)}(\approx+0.05 \mathrm{eV})$. Therefore, two holes are emitted by thermal activation form the $\mathrm{V}^{++}$state:

$$
V^{++} \stackrel{0.13 e V}{\longrightarrow} V^{+}+h \stackrel{0.05 e V}{\longrightarrow} V^{0}+2 h
$$

since the energy for emission of the first hole is larger than the energy for emission of the second one. Moreover, the energy required for a thermally activated vacancy diffusion is larger than the energy for a hole emission, and it depends on the charge state of the vacancy: $0.45 \mathrm{eV}$ for $\mathrm{V}^{0}, 0.32 \mathrm{eV}$ for $\mathrm{V}^{++}$and $0.18 \mathrm{eV}$ for $\mathrm{V}^{--}$(Table 3 ).

Thermally activated vacancies diffuse through a crystal lattice, and as mentioned previously, they can:

i. Recombine with silicon interstitial atoms;

ii. Interact with other atoms and form stable complexes with acceptors (B, Al, Ga), donors ( $\mathrm{P}, \mathrm{As}, \mathrm{Sb})$ or impurities (Ge, Sn, $\mathrm{H}, \ldots)$;

iii. Pair with other vacancies and form stable divacancies $\left(\mathrm{V}_{2}\right.$ or $\left.\mathrm{VV}\right)$ or small clusters of higher order vacancies $\left(\mathrm{V}_{3}, \mathrm{~V}_{4}, \ldots\right)$.

The di-vacancy is the amphoteric state that can be in one of four possible charge states $(--,-, 0,+)$, and beside the VO complex is the most prominent point defect formed by ionizing radiation in high resistivity (purity) single crystal silicon. Vacancy related defects are stable at the room temperature and have profound effect on electronic properties, such as the free carrier lifetime, the charge collection efficiency, the bulk generation current and the effective doping concentration in depletion region of silicon devices. The energy states of vacancy related defects for $n$-type $\mathrm{Si}$ are given in Table 3 . The di-vacancy $\left(\mathrm{V}_{2}\right)$ is thermally stable up to the temperature of $\sim 500 \mathrm{~K}$, while the vacancy oxygen (VO) complex is stable up to $\sim 550 \mathrm{~K}$ (Fig 40 ). The annealing temperature of vacancy related defects is $\sim 650 \mathrm{~K}$.

\section{Interstitial Atom Defects}

The interstiatial atom related point defects, e.g. the silicon atom interstial $(\mathrm{Si})_{\mathrm{i}}$, are second group of the most important defects in semiconductors, although only few defects have been characterized in detail. The most common interstitial defects in crystal silicon are shown in schematic temperature dependent diagram showing their 
thermodynamic properties (Fig 41). The thermal activation threshold temperature for diffusion of interstitials is $\sim 150 \mathrm{~K}$ [Wat92], i.e. at temperatures higher than the threshold value, such as the room temperature, interstials are highly mobile and can interact with other defects present in a silicon crystal lattice. Silicon interstials can exchange the supstitutional impurity atoms $\left(\mathrm{C}_{\mathrm{s}}, \mathrm{B}_{\mathrm{s}}, \mathrm{Al}_{\mathrm{s}}\right)$ and take their lattice site by means of the Watkins exchange mehanism. In high purity silicon the most important exchange process is the one involving an interstitional silicon and a substitutional carbon:

$$
(S i)_{i}+C_{s} \rightarrow(S i)_{s}+C_{i}
$$

Impurities left over at interstitial positions $\left(\mathrm{C}_{\mathrm{i}}, \mathrm{B}_{\mathrm{i}}, \mathrm{Al}_{\mathrm{i}}\right)$ are electrically active and mobile at room temperature. Additionally, an interstitial silicon atom can interact with an interstitial oxygen atom $\mathrm{O}_{\mathrm{i}}$, but this complex is unstable at the room temperature and decays quickly.

The vacancy - interstitial complex is the most interesting catagory of interstitial point defects in semiconductors from a perspective of the radiation damage caused by ionizing particles and photons, and in silicon example:

$$
V O_{i}+I \rightarrow O_{i} \text { or } V V+I \rightarrow V
$$

As an example, in a high resistivity $p$-type silicon used for production of silicon particle detectors, the boron concentration is low compared with the carbon concentration, so probabilty of an interaction between a primary defect and a boron atom is low compared to the formation of complexes involving silicon, carbon or oxygen atoms. The substitutional carbor is the main sink of mobile interstitional silicon atoms produced by ionizing radiation ( $\mathrm{Eq} \mathrm{75)}$. The newly formed interstitial carbon atom is mobile at room temperature and can interact further to produce defect complexes such as $\mathrm{C}_{i} \mathrm{O}_{i}$ and $\mathrm{C}_{i} \mathrm{C}_{s}$ (which are mostly associated with interstitial defects in high resistance silicon). Similary, the probability of a carbon phosphorus $\mathrm{C}_{\mathrm{i}} \mathrm{P}_{\mathrm{s}}$ complex formation in a high resistivity $n$ type silicon is also low because of the low phosphorous concentration compared to unwanted but everpresent carbon concentration $\left(\left[\mathrm{P}_{\mathrm{s}}\right] \sim 10^{12}-10^{13} \mathrm{~cm}^{-3}<<\left[\mathrm{C}_{\mathrm{s}}\right]<\left[\mathrm{O}_{\mathrm{s}}\right]\right)$.

\section{Effects of Radiation Damage on Electronic Properties of Semiconductor Devices}

Studies of the effects of radiation damage in semiconductor devices improve the understanding of the role played by a particular defect type on deterioration of electronic properties vital for operation of those devices, such as the reverse bias, the reverse current, the resistivity, the charge collection efficiency. Main theoretical concepts required for understanding of experimental studies are presented here, taking Si as an example.

\section{Carrier Generation Lifetime ( $\rightarrow$ Reverse current)}

Defects with energy levels close to the middle of the energy band gap are efficient generation/recombination centers of electron - hole pairs. These mid band gap defects produced by damaging ionizing radiation have a negative effect of increasing the bulk current, and consequently the reverse current through the $p-n$ or the Schottky junctions. The bulk current lbulk can be defined using the carrier generation lifetime $\mathrm{T} g$ :

$$
I_{b u l k}=A d q_{0} \frac{n_{i}}{\tau_{g}}
$$

where $A$ is the surface area and $d$ is the width of the depletion region, and $n_{i}$ is the intrinsic charge carrier concentration. Since different defects contribute to the generation of current, it is a sum of all contributions: 


$$
\tau_{g}=\left(\sum_{t} \frac{1}{\tau_{g, t}}\right)^{-1}=n_{i}\left(\sum_{t} G_{t}\right)^{-1}=n_{i}\left(\sum_{t} N_{t} \frac{e_{n, t} e_{p, t}}{e_{n, t}+e_{p, t}}\right)^{-1}
$$

The emission coeficients of electrons and holes $\left(e_{n}, e_{p}\right)$ for different defects $t$ (relation 69 is used for point defects) determine the probabilty of electron - hole pair generation $G$ $=\sum G_{t}$, i.e. the reverse current.

\section{Spatial Charge Density ( $\rightarrow$ Full depletion bias of junction semiconductor)}

Some stable defects created in the depletion region as a result of damaging ionizing irradiation contribute to the effective spatial charge density $N_{\text {eff }}$, and can have an effect on the full depletion voltage $V_{F D}, V_{F D} \sim\left|N_{\text {eff }}\right|$, (32). Ionized donors (donors occupied by a hole) contribute to the positive charge, while ionized acceptors (acceptors occupied by an electron) contribute to the negative charge in the depletion region. Therefore, by using formulae (47) for the trap occupancy in the depletion region, the effective spatial charge density in irradiated and partly damaged devices is:

$$
N_{\text {eff }}=\sum_{\text {donors in } S C R} p_{t}-\sum_{\text {acceptors in } S C R} n_{t}=\sum_{\text {donors }} N_{t} \frac{e_{n}}{e_{n}+e_{p}}-\sum_{\text {acceptors }} N_{t} \frac{e_{p}}{e_{n}+e_{p}}
$$

In silcon, donors in the upper half $\left(P_{s}\right)$ and acceptors in the lower half of the band gap $\left(B_{s}\right)$ are ionized in the depletion region at the room temperature. Donors in the lower half $\left(\mathrm{C}_{i} \mathrm{O}_{\mathrm{i}}\right)$ and acceptors in the upper half of the band gap $\left(\mathrm{VO}_{i}, \mathrm{C}_{i} \mathrm{C}_{s}\right.$, VV) (Fig 38) are not ionized at room temperature, and therefore do not contribute to the effective spatial charge density or changes to the full depletion reverse bias of the silicon semiconductor diode.

\section{Donor and Acceptor Carrier Removal by lonizing Radiation ( $\rightarrow$ Reverse bias of semiconductor)}

Ionizing radiation produce mobile primary defects which interact with doping impurities like the phosphorous or boron atoms in $\mathrm{Si}$, forming defect complexes or removing impurities from their lattice sites. Examples are the following processes:

$$
V+P_{S} \rightarrow V P_{S} \text { and } / \text { or } I+B_{z} \rightarrow B_{i}
$$

Newly formed $\mathrm{VP}_{\mathrm{s}}$ and $\mathrm{B}_{\mathrm{i}}$ defects are not ionized in the depletion region, so the effective spatial charge density $N_{\text {eff }}$ is decreased. These processes of shallow acceptor or donor removal have important effect on changes to the increase of depletion width (for constant bias), the capacitance decrease, and the reverse bias decrease, as it will be shown on the example of silicon.

\section{Free Charge Carrier Concentration ( $\rightarrow$ Resistivity)}

The semiconductor resistivity $\rho$ is defined as:

$$
\rho=\frac{1}{\sigma}=\frac{1}{q\left(\mu_{n} n+\mu_{p} p\right)}
$$

where $\sigma$ is the conductivity, $n$ and $p$ are concentrations of free charge carriers (electrons and holes) in thermal equilibrium, and $\mu_{n}$ and $\mu_{p}$ are the electron and the hole mobilty. In a case of a prisitne (non-irradiated) material, the concentration of free charge carriers is determined by concentration of shallow doping atoms. Therefore, the resistivity can 
be expressed using the effective spatial charge concentration $N_{\text {eff, }}$ or the effective doping of semiconductor:

$$
\rho=\frac{1}{\sigma}=\frac{1}{q_{0} \mu_{n, p}\left|N_{e f f}\right|}
$$

where $N_{\text {eff }}=N_{\text {donor }}-N_{\text {akceptor }}$ and $\mu_{n, p}$ is the mobility of majority charge carriers. This expression is only valid in a case of small changes to the effective doping caused by the carrier removal in partly damaged semiconductors. The resistivity is related to the serial resistance $R_{s}$ of non-depleted (neutral) semiconductor of the thickness $d-W$ and the surface area $A$ in following manner:

$$
R_{s}=\rho \frac{(d-W)}{A}
$$

From expression (83) it can be seen that by changing the effective free charge carrier density, the irradiation of a semiconductor changes its resistance.

\section{Free Charge Carrier Trapping ( $\rightarrow$ Charge collection efficiency)}

In general, the capture of free charge carriers by deep traps has a profound effect on the carrier lifetime and consequently the charge collection efficiency in semiconductors. Free electrons and holes produced by ionizing particles and photons move under the influence of applied electric field (drift) in the depletion region of reversely biased device. The charge carrier drift motion in the electric filed induces the carrier current. The charge collection is a process of charge induction on collecting electrodes of the semiconductor device. The induced charge value corresponds to the integral of induced current. In a case of irradiated and partly damaged semiconductor, deep defects produced by ionizing radiation trap a part of drifting free charge carriers. Trapped carriers do not contribute to the induced current, and if not released quickly (short trapping time) during the characteristic time of charge integration, they do not contribute to the collected charge. The net result is the decreased charge collection efficiency in partly damaged (low level damage) semiconductor devices. The capture probability of free electrons $1 / T_{t, n}$ (holes $1 / T_{t, p}$ ) in different traps $(t)$ is proportional to the electron (hole) capture coefficient in a given defect $c_{n, t}\left(c_{p, t}\right)$, the defect concentration $N_{t}$ and the ratio of occupied states to the total number of possible states:

$$
\frac{1}{\tau_{t, n}}=\sum_{t} c_{n, t} N_{t} \frac{e_{n, t}}{e_{p, t}+e_{n, t}}, \frac{1}{\tau_{t, p}}=\sum_{t} c_{p, t} N_{t} \frac{e_{p, t}}{e_{p, t}+e_{n, t}}
$$

A starting assumption for studies and modelling/interpretation of the deterioration of charge collection efficiency in the low level damage approximation limit is that the carrier capture probability in the irradiated semiconductor $1 / \mathrm{T}$, increases linearly with the formation of new traps in comparison to a pristine semiconductor material $1 / \tau_{0}$ [52], i.e:

$$
\frac{1}{\tau}=\frac{1}{\tau_{0}}+N_{t} \sigma v_{t h}
$$

where $N_{t}$ is the concentration of additional electrically active traps produced by ionizing radiation, $\sigma$ is the charge carrier capture cross section (usually in the $10^{-14} \mathrm{~cm}^{-2}-10^{-16}$ $\mathrm{cm}^{-2}$ range for above- mentioned traps in silicon $([53,48]), U_{\text {th }}$ is the thermal velocity of a charge carrier, and $T_{0}$ is the carrier lifetime in a pristine material. In high resistivity silicon the lifetime of charge carriers is of the order of $10 \mu \mathrm{s}$ [26], and the following 
relation holds:

$$
\frac{1}{\tau_{t, n}}=\frac{1}{\tau_{t, p}}=\frac{1}{\tau_{0}}
$$

In an example of lon Beam Induced Charge (IBIC) measurements on Si PIN photodiode, which is described as a layer of almost intrinsic silicon n-type, and for which the blocking voltage is high enough to achieve the electric field strength of a dozen of $\mathrm{kV} / \mathrm{cm}$, the rate of charge of carrier saturation is of the order of $10^{7} \mathrm{~cm} / \mathrm{s}[26]$.

The equation (85) is one of the most important assumptions of the IBIC model, which uses the equations of charge carrier motion presented and the NIEL concept presented earlier to interpret and to simulate the experimentally measured decrease of charge collection efficiency as a function of particle/ion fluence.

\section{Example of Characterisation of Small-scale Defect in Semiconductors I-V Characteristics}

The dominant current trasport mechanism in a semiconductor diode is the thermal emission of the majority carriers over potential barriers established between the metal and semiconductor (Schottky diode) or two semiconductors of different types of conductivity ( $p-n$ diode). A quantitative analysis shows that the ideal current-voltage characteristics has the form:

$$
I=I_{S}\left(\exp \left(\frac{e V}{k_{B} T}\right)-1\right)
$$

where $V=V_{F}>0$ in forward bias and $V=-V_{R}<0$ in reverse bias. The saturation current Is is given by:

$$
I_{S}=A A^{*} T^{2} \exp \left(\frac{-e \Phi_{B n}}{k_{B} T}\right)
$$

where $A$ is the diode surface area and $A^{*}$ is the effective Richardson constant. An example of the mesaured I-V characteristic for a semiconductor diode is shown in Fig 42. A saturating component of the total current (shown in red) correspond to the bulk generation current.

\section{C-V Characteristics}

The capacitance $\mathrm{C}$ associated with the depletion region is defined as:

$$
C=\frac{\varepsilon \varepsilon_{0} A}{W}
$$

where $A$ is the area and $\mathrm{W}$ is the width of the depletion region. The capacitance-voltage characteristic $(C(V))$, an example shown in Fig 43, allows determination of the spatial distribution of free charge carriers (Fig 44), i.e. active dopants, in a semiconductor (both pristine or irradiated or partly damaged) using the following equation:

$$
N_{D}^{*}(W)=\frac{2}{e \varepsilon \varepsilon_{0} A^{2}} \cdot \frac{1}{\frac{d}{d V}\left(\frac{1}{c^{2}}\right)}
$$




\section{Capacitive Transient Techniques}

\section{Deep Level Transient Spectroscopy (DLTS)}

Deep level transient spectroscopy is as a unique and powerful tool for the study of electrically active defects in semiconductors. The basis of DLTS is the repetitive filling and emptying of deep defects in the depletion region of a semiconductor by a bias pulse, as schematically show in Fig 45.

The resulting capacitance transient of a diode is filtered electronically and monitored as a function of the sample temperature. The semiconductor sample ( $p$ - $n$ junction or Schottky diode) is operated under reverse bias $V_{R}$, which during an applied bias pulse is reduced to $V_{P}$. The empty traps residing in the former depletion region will be able to capture free carriers (electons in $n$-type and holes in $p$-type semiconductor) and become occupied. After restoration of the original bias $V_{R}$, the charge in the depletion region will be lower then before the forward bias (majority carrier) pulse was applied, due to the carriers stored in the deep defects. These carriers will be released again through thermal emission, which proceeds exponentially in time, with a time constant $\tau_{n, p}$ which is given by:

$$
\tau_{n, p}=\frac{1}{K_{T} T^{2}} \exp \left(\frac{E_{T}}{k T}\right)
$$

This thermal discharging of the occupied traps is registered by measuring the capacitance of the reverse biased diode as a function of time after the filling pulse has been applied. In the first instance, for a low defect concentration, the resulting capacitance transient proceeds exponentially in time, having the same time constant:

$$
\Delta C(t) \equiv C(t)-C(\infty)=-\Delta C \exp \left(-\frac{t}{\tau_{n, p}}\right)
$$

where $\Delta C$ is the absolute value of the capacitance transient amplitude and $C(\infty)$ is the steady-state capacitance for $t$ going to $\infty$. The amplitude of the capacitance transient is in proportion to the trap concentration. The sign of the amplitude is negative for the majority carrier emission, and positive for the minority carrier emission.

As mentioned at the beginning, the main feature of DLTS is to monitor capacitance transients as a function of the sample temperature. In the original system proposed by D.V. Lang [54], the capacitance transient is measured at two fixed times $t_{1}$ and $t_{2}$ after the pulse and the difference of two capacitance values $\left(\Delta C=C\left(t_{1}\right)-C\left(t_{2}\right)\right)$ is plotted. Fig. 46 shows schematically how the peak- shaped DLTS signal will appear when the temperature is varied. The peak maximum corresponds to a time constant $I_{\max }$ defined by the selected instrumental values $t_{1}$ and $t_{2}$ :

$$
\tau_{\max }=\left(t_{1}-t_{2}\right)\left\{\operatorname{Ln}\left(\frac{t_{1}}{t_{2}}\right)\right\}
$$

The corresponding emission rate $e_{\max }=T_{\max }{ }^{-1}$ is often called the emission rate window. Changing $t_{1}$ and/or $t_{2}$ will change $e_{\max }$ and hence the peak position $T_{\max }$ corresponding to a certain deep level $E_{T}$. For each deep level present in a characterized material above a minimum concentration limit, a DLTS peak will show up. The detection limit of capacitance transient spectroscopy is approximately $10^{-5}$ times the background free carrier concentration, i.e. for typical doping concentrations of $10^{13}-10^{16} \mathrm{~cm}^{-3}$ the low level detection limit is $10^{8}-10^{11}$ defects per $\mathrm{cm}^{-3}$ [55], several orders of magnitude better than 
most of other techniques used for defect characterisation in semiconductors.

A complete analysis of DLTS data yields the following information: (1) the defect state concentration, (2) the activation energy for the electronic transition, (3) the majority carrier capture cross section and (4) the depth distribution of the defect.

\section{Activation Energy}

A set of DLTS peak maxima $T_{\max }$ can be obtained if the temperature scans for different rate windows are repeated. Activation energy of electron and hole emission can be obtained from the slope of an Arrhenius plot of $T^{2}$-corrected electron and hole emission rates. The intercept is proportional to the majority carrier capture cross section. Fig 47 shows as example one typical Arrhenius plot.

\section{Capture Cross Section}

The capture cross section of a defect for majority carriers is determined experimentally by measuring the height of the DLTS signal as a function of the duration of the filling pulse at a constant temperature. The signal must saturate at long filling pulses, corresponding to the complete occupation of defects with majority carriers. Plotting In[(saturated maximum signal) -(signal at pulse width equal to $t$ )] vs pulse width $t$ should yield a straight line for point-like defects, with the slope value equal to the capture rate c:

$$
c=n \sigma_{n} v_{t h}
$$

Based on the observed kinetics (exponential or logaritmic), it is possible to distinguish whether a defect is a point-like or extended (cluster). Fig $\mathbf{4 8}$ shows DLTS signal as a function of the pulse width.

Additionally, from the magnitude of the capture cross section it is possible to determine the nature of a trap, i.e. whether it is a Coulombic attractive trap for electrons (deep donor in n-type) or holes (deep acceptor in p-type material), but only for traps with the capture cross section value above $10^{-14} \mathrm{~cm}^{-2}$ (neutral traps have a cross section value in the range $10^{-15} \mathrm{~cm}^{-2}$ ). Deep levels with cross section values below or in the $10^{-16} \mathrm{~cm}^{-2}$ range can be considered repulsive for the majority carriers,

i.e. they correspond to deep donors in p-type and deep acceptors in n-type material.

An alternative way to distinguish the charge state of a defect is by studying the electrical field dependence of the activation energy.

\section{Depth Profiling}

The DLTS signal from defects is directly related to their concentration $N_{T}$ in the small signal regime, where the number of defects is much less than the free carrier concentration, which can be determined from a $\mathrm{C}-\mathrm{V}$ measurement. The defect concentration $N_{T}$ can be determined using:

$$
N_{T}=2 N_{D} \frac{\Delta C}{C_{0}}
$$

where $\Delta C$ is measured from the DLTS peak amplitude and $C_{0}$ is the capacitance of the sample at the peak temperature and reverse bias $V_{R}$. If combined with $\mathrm{C}-\mathrm{V}$ measurements, the DLTS technique can be used for determination of the defect profile as a function of the depletion width. The most common way to do it is to measure the 
incremental change of the DLTS signal, which corresponds to a small change in the measurement pulse amplitude by keeping constant the reverse bias applied to a sample. This procedure becomes increasingly difficult for shallow junctions, where the fundamental limit of resolution is the Debye length. Debye length defines a small transition region at the boundaty between the depletion region and the electrically neutral region, and it is only partly depleted of free charge carriers. DLTS technique works well under the assumption that no free charge carriers exist within the depletion region and that the boundary to the electrically neutral region is sharp. This assumption is known as the depletion approximation, and it is satisfied when the width of the depletion region is much larger tha the Debye length. It has been shown in many studies that the numerically corect estimation of the defect concentration $\lambda$-effect (the position where the Fermi level and deep level are crossing over) should be taken into account. Zohta et al

[56] have introduced correction as:

$$
f(W)=\left(W_{0}-\lambda_{0}\right)^{2}-\left(W_{1}-\lambda_{1}\right)^{2} / W_{0}^{2}
$$

where $W_{0}$ and $W_{1}$ are depletion width for reverse bias and pulse, while $\lambda$ is defined as:

$$
\lambda=L_{D} \sqrt{2}\left[\operatorname{Ln}\left(\frac{c_{n} N_{D}}{e_{n}}\right)\right]^{1 / 2}
$$

where $L_{D}$ is Debye length, defined as:

$$
L_{D}=\sqrt{\frac{\varepsilon_{0} \varepsilon k T}{e^{2} N_{D}}}
$$

The final formula for a defect concentration can now be written as:

$$
N_{T}=2 \cdot \frac{\Delta C_{0}}{C} \cdot N_{D} \cdot \frac{1}{f(W)}
$$

In summary, DLTS is an experimental technique that produces a sequence of peaks as the temperature is scanned. Each peak can be interpreted as related to an electrically active defect. In other words, it is a filtering technique in which a peak is produced when the emission rate matches the filter rate window. In general, thermal emission transients from deep level states are often small and superimposed on a background potential that changes slowly as the temperature is scanned. As a consequence the fundamental requirement for any DLTS system is a rejection of this background level [57].

\section{Charge Collection Efficiency Technique}

Irradiation of a semiconductor junction with an arbitrary dose of any ionizing radiation deteriorates its performance, due to the creation of defects, as mentioned above. Using a micro-beam and the IBIC technique described earlier, the effects of radiation damage on free charge carrier properties and charge collection efficiency (CCE) in semiconductor junctions can be characterised $[52,58,59,37,60,61,30,31,32,35$, 62].

Here the example is given for a PN junction, with the probing ion beam being $\mathrm{H}$ or $\mathrm{He}$ ions, which are best suited for characterization by the IBIC microscopy because the nonionizing energy loss is the lowest for light ions and the resulting excess damage due to IBIC characterization is negligible. The CCE degradation is monitored as a function of damaging particle or ion beam (DIB) fluence. DIB can be any ion with desired properties 
like the appropriate range in a material (device) or the primary defect introduction rate depth profile. The model takes following processes into account:

i) The final defect formation from initial lattice atom displacements caused by ion projectiles and secondary recoiling target atoms;

ii) The nature of the ion probe interaction with partly damaged junction (device);

iii) The charge collection mechanism of free carriers generated by probing ions, which depends on the electrostatics of the device.

All these processes can be integrated into a unique model [31] based on the theory of IBIC technique [63,64]. The basic assumption for the validity of this model is the "quasisteady state approximation", in which the free charge carriers generated by the single ion probe interaction with semiconductor material do not significantly perturb the electrostatic field within the tested junction. This assumption is equivalent to the statement that free carriers, electrons $(n)$ and holes $(p)$, generated by ionization, move in a static electric field given by the solution of the steady state basic equations of semiconductors. The total induced charge $Q_{s}(t)$ is then given by the superposition of the individual electron and hole contributions [Jac75]:

$$
Q_{S}(t)=\int_{\Omega} d^{3} \rho\left[Q_{n}(t ; \vec{\rho})+Q_{p}(t ; \vec{\rho})\right] \Gamma(\vec{\rho})
$$

where $Q_{n, p}$ is the collected charge at the sensitive electrode induced by the motion of electrons and holes, respectively, $\Omega$ is the active volume of the junction and $\Gamma$ is the profile distribution of generated defects.

By virtue of the quasi-steady state approximation, no interaction between excess charge carriers is taken into account, i.e. we assume the plasma and/or high charge injection effects to be negligible. As a consequence, this approximation allows the decoupling of the electrostatics (Poisson's equation) and the carrier continuity equations. It follows that the $Q_{n} / Q_{p}$ terms in eq. (100) can be considered as the sum of the individual contributions of electrons/holes moving separately within the static electric field region.

The instantaneous current (is) induced at the sensitive electrode $S$ by the motion of an elementary charge $\mathrm{q}$ with a drift velocity $\mathrm{v}$ can be calculated by means of a general formula, introduced by

J.B. Gunn [65]:

$$
i_{S}=-q \cdot \vec{v} \cdot \frac{\partial \vec{F}}{\partial V_{s}}
$$

$\frac{\partial \vec{F}}{\partial V_{s}}$ term is the "Gunn's weighting field", which is defined as the partial derivative of the actual electric field $(F)$ with respect to the bias voltage $V_{S}$ applied to the sensing electrode $\mathrm{S}$, while the voltage is kept constant on all the other electrodes.

The time integral of eq. (101) gives the charge qS $(t ; \rho)$ induced at the sensing electrode at time $t$ by a point charge q generated at $t=0$ in a position $r=\rho$ :

$q_{s}(t ; \vec{\rho})=\int_{0}^{t} d t^{\prime} i_{s}\left(t^{\prime}\right)=-q \cdot \int_{0}^{t}\left[\vec{v} \cdot \frac{\partial \vec{F}}{\partial V_{s}}\right]=-q \cdot \int_{\vec{\rho}}^{r(t)} \frac{\partial \vec{F}}{\partial V_{s}} \cdot d \vec{l}=q \cdot\left[\left.\frac{\partial \psi}{\partial V_{s}}\right|_{r(t)}-\left.\frac{\partial \psi}{\partial V_{s}}\right|_{\vec{\rho}}\right]$

where $\psi$ is the actual electrostatic potential (i.e. $F=-\nabla \psi$ ), $r(t)$ is the position of the moving charge at time $t$, and the line integral is calculated along the carrier trajectory. The induced charge in the case of moving single point charge is then simply given by the difference of the "Gunn's weighting potential" $\frac{\partial \psi}{\partial V_{s}}$ between the final and initial positions of the point charge. A method to calculate the weighting field is given in the work by L. Grassi et al. [66]. 
The total charge $Q_{n, p}$ induced at the sensing electrode at time $t$ by the motion of electrons and holes generated at a certain positon $\rho$, is obtained by combining Eq.(102) with Eq.(100):

$$
Q_{n}(t ; \vec{\rho})+Q_{p}(t ; \vec{\rho})=-q \cdot \int_{0}^{t} d t^{\prime} \int_{\Omega} d^{3} r\left\{\left[n\left(\vec{r}, t^{\prime} ; \vec{\rho}\right) \cdot \overrightarrow{v_{n}}(\vec{r})+n\left(\vec{r}, t^{\prime} ; \vec{\rho}\right) \cdot \overrightarrow{v_{p}}(\vec{r})\right] \cdot \frac{\partial \vec{F}}{\partial V_{s}}\right\}
$$

where $\mathrm{n}$ and $\mathrm{p}$ are the excess charge concentrations of electrons and holes, respectively, whose spatial-temporal evolution can be calculated by solving the electron/hole continuity equation, with

the initial condition: $n(r, t=0 ; \rho)=p(r, t=0 ; \rho)=\delta(r-\rho)$.

It is important to understand that decoupling of the electron and hole contributions in Eq. (103) implies not only the negligible interaction of electrons and holes, but also the linear superposition of the electron/hole recombination/trapping processes. As a consequence, the electron and hole continuity equations contain only a linear term and the "adjoint model" proposed by T.H. Prettyman [67] can be effectively adopted.

In addition, if the free carrier generation terms are defined in the following manner:

$$
\begin{gathered}
G_{n}(\vec{r})=\overrightarrow{v_{n}}(\vec{r}) \cdot \frac{\partial \vec{F}}{\partial V_{s}}-\nabla\left[D_{n} \cdot \frac{\partial \vec{F}}{\partial V}\right] \\
G_{p}(\vec{r})=\overrightarrow{v_{p}}(\vec{r}) \cdot \frac{\partial \vec{F}}{\partial V_{s}}-\nabla\left[D_{p} \cdot \frac{\partial \vec{F}}{\partial V}\right]
\end{gathered}
$$

Then the two terms in the eq. (103) are the Green's function of the carrier (electron or hole) continuity equations.

As a result of the adjoint model formalism, the contribution of electrons and holes to the total induced charge can be evaluated by solving the adjoint equations of the relevant continuity equations:

$$
\frac{\partial \xi^{+}}{\partial t}=(-1)^{(\alpha+1)} \cdot \overrightarrow{v_{\xi}} \cdot \vec{\nabla} \xi^{+}+\nabla\left(D_{\xi} \cdot \vec{\nabla} \xi^{+}\right)-R+G_{\xi, S}^{+}
$$

where $\xi^{+}$is the adjoint function for electrons $\left(\xi^{+}=n^{+} ; \alpha=0\right)$ or for holes $\left(\xi^{+}=p^{+} ; \alpha=1\right), D_{n, p}$ is the diffusion coefficient of electrons or holes, and $R$ is the linearized recombination term. Assuming homogeneous boundary and initial conditions, it can be demonstrated [Pre99] that the charge at the sensitive electrode induced by the motion of free carriers generated at the starting point $\rho$ is given by:

$$
Q_{n, p}(t ; \vec{\rho})=q \cdot\left(n^{+}(\vec{\rho}, t)+p^{+}(\vec{\rho}, t)\right)
$$

The recombination term $R$ in eq. (105) can be linearized if i) the excess free carrier concentration is small compared to the number of available recombination centres and ii) de-trapping time is small compared to the carrier transit time from any generation point to the collecting electrode. In this case, R is proportional to the carrier concentration:

$$
R_{n}=\frac{n}{\tau_{n}} ; R_{p}=\frac{p}{\tau_{n}}
$$


where $\mathrm{T}_{n, p}$ are the electron and hole lifetime, respectively.

\section{IBIC CHARACTERIZATION OF A PARTLY DAMAGED SC DIODE - A NEW MODEL FOR CALCULATION OF CCE IN PRESENCE OF DEEP TRAPS}

In describing the IBIC characterisation of a partly damaged semiconductor junction, we make use of the Shockley-Read-Hall theory of semiconductors in thermodynamic equilibrium, and according to it, the carrier lifetime is related to the concentration of recombination centres $\mathrm{N}^{\top}$ through the following expression [68]:

$$
\frac{1}{\tau_{n, p}}=N_{n, p}^{T} \cdot \sigma_{n, p} v_{n, p}^{t h}
$$

where $v_{n, p}^{\text {th }}$ and $\sigma_{n, p}$ are the carrier thermal velocities and the carrier capture cross sections for electrons ( $n$ ) and holes (p), respectively.

Eq. (108) allows the effect of radiation damage to be integrated in the unique radiation damage model by assuming a proportionality of the recombination centre concentration $\mathrm{N}^{\top}$ and the concentration of vacancy-interstitial Frenkel pairs (FP) as primary point defects. It follows that the carrier lifetime distributions can be related to the recombination parameters using the following equation [29]:

$$
\frac{1}{\tau_{n, p}(\vec{r})}=N_{n, p}^{T 0} \cdot \sigma_{n, p}^{0} \cdot v_{n, p}^{t h}+N_{n, p}^{T} \cdot \sigma_{n, p} \cdot v_{n, p}^{t h}=\frac{1}{\tau_{n, p}^{0}}+K_{n, p}(\vec{r}) \cdot \Phi
$$

where the superscript "o" denotes the terms relevant to the pristine material $\left(\tau_{n, p}^{0}\right.$ is assumed constant), $\tau_{n, p}$ is the carrier lifetime after the irradiation with the fluence $\Phi$ of damaging ions which generate the concentration of recombination centres $N^{\top}(r)$ with the capture cross section $\sigma_{n, p} ; \mathrm{K}$ is the (recombination) lifetime damage coefficient defined as [62]:

$$
K_{n, p}(\vec{r})=\frac{N_{n, p}^{T}(\vec{r}) \cdot \sigma_{n, p} \cdot v_{n, p}^{t h}}{\Phi}
$$

Furthermore, under the previous assumption of $\mathrm{N}^{\top}$ and FP proportionality, the concentration of active recombination centres $N_{n, p}^{T}$ scales with the damaging ion fluence $\Phi$. The proportionality factor is given by the product of the vacancy concentration distribution per ion, i.e. the vacancy distribution profile, $V(r)$ and the average number of active defects (carrier traps) generated by a single vacancy $\mathrm{k}$ :

$$
N_{n, p}^{T}(\vec{r})=k_{n, p} \cdot V(\vec{r}) \cdot \Phi \Rightarrow K_{n, p}(\vec{r})=k_{n, p} \cdot V(\vec{r}) \cdot \sigma_{n, p} \cdot v_{n, p}^{t h}
$$

The vacancy distribution profile $V(r)$ can be calculated in the binary collisions approximation (BCA) by the SRIM code [20] or in the molecular dynamics approximation by the Marlowe code [69].

In the studied case of a particle detector with the planar geometry, we can further simplify the problem by reducing it to only one dimension (depth) by the perpendicular irradiation of a planar device and the assumption that the trajectories of high energy ion projectiles (in the $\mathrm{MeV}$ energy range) in a material can be considered nearly straight lines, i.e. the end of an ion range is much larger than the lateral straggling of a light ion producing FP directly. Moreover, using a raster- scanned ion microbeam with micrometre spatial resolution for the vacancy production (ion projectiles entering the 
surface of a device within a pixel of $1 \mu \mathrm{m}$ by $1 \mu \mathrm{m}$ size with maximum angular deviation from normal incidence $\pm 1^{\circ}$ ), the vacancy distribution generated along ion cascade by secondary recoils deviating from the straight line direction of the projectile is averaged. It is important that due to irradiation conditions with the average ion beam rate up to $10 \mathrm{kHz}$ and the pixel dwell time of $500 \mu \mathrm{s}$, we can assume that defects are formed in the individual cascades well separated both temporarily and spatially. The cumulative effect of multiple scanning of the region of interest to create a damage structure (usually $100 \mu \mathrm{m} \times 100 \mu \mathrm{m}$ ) is the uniform cross sectional areal distribution of finally formed defects. As a consequence, we need to consider the generation of vacancies along the direction normal to the irradiated electrode (i.e. x-direction). Similarly, the resultant carrier generation volume, which is nearly cylindrical shape with a diameter of tens of nanometres [70], can be assumed to be a function of only the $x$ coordinate and proportional to the ionization energy loss profile as:

$$
\frac{d E_{I}}{d x}=\Gamma(x) \cdot \varepsilon_{n, p}
$$

where $\varepsilon n p$ is the average energy required to create electron/hole pairs (in silicon $3.6 \mathrm{eV}$, [71]), $\mathrm{El}$ is the energy of the ion probe, and $\frac{d E_{I}}{d x}$ is the relevant ionization energy loss that can be extracted from SRIM simulations, as shown in Fig. 25 for the ions used.

Moreover, if we consider that in our experimental conditions all carrier generation and recombination processes occur within the depletion region of a device, the dominant charge transport mechanism is the carrier drift caused by the applied electric field whose direction is perpendicular to the electrodes. As a consequence of the carrier generation, and the transport and recombination within the depleted region, a diffusion of charge carriers is reasonably assumed to be negligible and the "Gunn's weighting potential" assumes the form of the conventional weighting potential as defined by the "ShockleyRamo" theorem $[72,63]$.

Schematics of a one-dimensional geometry junction is outlined in Fig. 49. The cathode is located at $\mathrm{x}=0$ and the anode located at

In these conditions the adjoin equations \#(105) can be re-written as the following first order space- time differential equations [62]:

$$
\left\{\begin{array}{l}
\frac{\partial n^{+}(x, t)}{\partial t}=-v_{n}(x) \cdot \frac{\partial n^{+}(x, t)}{\partial x}-n^{+}(x, t) \cdot\left(\frac{1}{\tau_{n}^{0}}+k_{n} \cdot V(x) \cdot \sigma_{n} \cdot v_{n}^{t h}\right)+v_{n}(x) \cdot \frac{\partial \vec{F}(x)}{\partial V_{s}} \\
\frac{\partial p^{+}(x, t)}{\partial t}=+v_{p}(x) \cdot \frac{\partial p^{+}(x, t)}{\partial x}-p^{+}(x, t) \cdot\left(\frac{1}{\tau_{p}^{0}}+k_{p} \cdot V(x) \cdot \sigma_{n} \cdot v_{p}^{t h}\right)+v_{p}(x) \cdot \frac{\partial \vec{F}(x)}{\partial V_{s}}
\end{array}\right.
$$

With homogeneous initial $(\mathrm{t}=0)$ and boundary conditions defined at the irradiated (at $\mathrm{X}=0$ ) and back (at $\mathrm{x}=\mathrm{d}$ ) electrodes:

$$
\left\{\begin{array}{l}
n^{+}(x=d, t)=n^{+}(x=0, t)=n^{+}(x, t=0)=0 \\
p^{+}(x=d, t)=p^{+}(x=0, t)=p^{+}(x, t=0)=0
\end{array}\right.
$$

Usually in the experiment only the total induced charge is considered, so the time dependent adjoin equations 0 will asymptotically converge to the following steady state equations:

$$
\left\{\begin{array}{l}
v_{n}(x) \cdot \frac{\partial n^{+}(x, t)}{\partial x}=-n^{+}(x, t) \cdot\left(\frac{1}{\tau_{n}^{0}}+k_{n} \cdot V(x) \cdot \sigma_{n} \cdot v_{n}^{t h}\right)+v_{n}(x) \cdot \frac{\partial \vec{F}(x)}{\partial V_{s}} \\
-v_{p}(x) \cdot \frac{\partial p^{+}(x, t)}{\partial x}=-p^{+}(x, t) \cdot\left(\frac{1}{\tau_{p}^{0}}+k_{p} \cdot V(x) \cdot \sigma_{n} \cdot v_{p}^{t h}\right)+v_{p}(x) \cdot \frac{\partial \vec{F}(x)}{\partial V_{s}}
\end{array}\right.
$$

With the following boundary conditions: 


$$
\left\{\begin{array}{l}
n^{+}(x=d)=0 \\
p^{+}(x=d)=0
\end{array}\right.
$$

Their solution is given by the following expressions:

$$
\left\{\begin{array}{l}
n^{+}(x)=\int_{x}^{d} d y \cdot \frac{\partial \vec{F}(y)}{\partial V_{s}} \cdot \exp \left[-\int_{x}^{y} d z\left(\frac{1}{v_{n}(z) \cdot \tau_{n}^{0}}+\Phi \frac{k_{n} \cdot V(z) \cdot \sigma_{n} \cdot v_{n}^{t h}}{v_{n}(z)}\right)\right] \\
p^{+}(x)=\int_{0}^{x} d y \cdot \frac{\partial \vec{F}(y)}{\partial V_{s}} \cdot \exp \left[-\int_{y}^{x} d z\left(\frac{1}{v_{p}(z) \cdot \tau_{p}^{0}}+\Phi \frac{k_{p} \cdot V(z) \cdot \sigma_{p} \cdot v_{p}^{t h}}{v_{p}(z)}\right)\right]
\end{array}\right.
$$

In conclusion, the solution of the steady state equations (115), integrated in eq. (117) provides the following expression of the charge collected at the sensitive electrode:

$$
Q_{S}=\int_{0}^{d} d x \cdot \Gamma(x)\left\{\begin{array}{l}
\int_{x}^{d} d y \cdot \frac{\partial \vec{F}(y)}{\partial V_{s}} \cdot \exp \left[-\int_{x}^{y} d z\left(\frac{1}{v_{n}(z) \cdot \tau_{n}^{0}}+\Phi \frac{k_{n} \cdot V(z) \cdot \sigma_{n} \cdot v_{n}^{t h}}{v_{n}(z)}\right)\right]+ \\
\int_{0}^{x} d y \cdot \frac{\partial \vec{F}(y)}{\partial V_{s}} \cdot \exp \left[-\int_{y}^{x} d z\left(\frac{1}{v_{p}(z) \cdot \tau_{p}^{0}}+\Phi \frac{k_{p} \cdot V(z) \cdot \sigma_{p} \cdot v_{p}^{t h}}{v_{p}(z)}\right)\right]
\end{array}\right\}
$$

Equation (118) is a general expression, whose validity stems from the assumption of low radiation damage, which is described by the linearized recombination terms given by the eqs. (107) -(111).

Finally, normalizing $Q_{s}$ by the charge induced in the pristine sample $Q_{0}$, the expression for the charge collection efficiency as a function of the damaging ion beam fluence $\Phi$ is [62]:

$$
C C E=\int_{0}^{d} d x \cdot \gamma(x)\left\{\int_{x}^{d} d y \cdot \frac{\partial \vec{F}(y)}{\partial V_{s}} \cdot \exp \left[-\int_{x}^{y} \frac{d z}{\lambda_{n}(z)}\right]+\int_{0}^{x} d y \cdot \frac{\partial \vec{F}(y)}{\partial V_{s}} \cdot \exp \left[-\int_{y}^{x} \frac{d z}{\lambda_{p}(z)}\right]\right\}
$$

where we have assumed that the induced charge is totally collected in the pristine device,

And the normalized ionization energy loss profile and $\lambda$ is the mean free path (drift length) of carriers:

$$
\gamma(x)=\frac{1}{E_{I}} \cdot \Gamma(x)
$$

And the normalized ionization energy loss profile and $\lambda$ is the mean free path (drift length) of carriers:

$$
\frac{1}{\lambda_{n, p}(z)}=\frac{1}{\lambda_{n, p}^{0}(z)}+\Phi \cdot k_{n, p} \cdot \frac{V(z) \cdot \sigma_{n, p} \cdot v_{n, p}^{t h}}{v_{n, p}(z)}
$$

Where $\lambda_{n, p}^{0}(z)=v_{n, p}(z) \cdot \tau_{n, p}^{0}$ is the carrier drift length in the pristine material, which is much longer than $\mathrm{d}$.

Borrowing the basic concepts from the scattering theory [73], the integrands at the exponent of the nested integrals in eq. 0 have the meaning of the probability $P_{n, p}$ that a carrier will be stopped in a slab of infinitesimal thickness $\mathrm{dz}$ :

$$
P_{n, p}(z) \cdot d z=\frac{d x}{\lambda_{n, p}(z)}=\rho_{n, p}(z) \cdot \sigma_{n, p}^{e f f}(z) \cdot d z=\left[\Phi \cdot k_{n, p} \cdot V(z)\right] \cdot\left(\sigma_{n, p} \cdot \frac{v_{n, p}^{t h}}{v_{n, p}(z)}\right) \mathrm{dz}
$$

Where $\rho(z)=\Phi \cdot k_{n, p} \cdot V(z)$ is the density of recombination centers of effective cross section $\sigma_{n, p}^{e f f}(z)=\left(\sigma_{n, p}(z) \frac{v_{n, p}^{t h}}{v_{n, p}(z)}\right)$ present in the slab.

It is worth noticing that $\sigma_{n, p}^{e f f}$ is inversely proportional to the carrier drift velocity, i.e. it decreases as the velocity increases. This conclusion resembles the $(1 / \mathrm{v})$ law relevant to the neutron capture by a free nucleus (if the complex scattering length for neutron-nucleus interaction is constant) [73] and its simple interpretation can be adopted: as the carrier velocity decreases, the carrier lingers for a longer time in the neighborhood of the recombination center, which has a longer time to capture a charge carrier. 
Although eq.119 provides the solution for the degradation problem in CCE, its complex analytical expression can obscure its physical meaning. In the following we will discuss briefly some more transparent cases and simple (asymptotic) solutions of Eq.119 which are routinely used and described in the paper by Vittone et al [62]

\section{Case I: the Hecht's relation}

In this case, the following assumptions are made:

i) A constant vacancy profile throughout the entire device $V(z)=\frac{V_{T}}{d}$, where $\mathrm{V}_{\mathrm{T}}$ is the total number of vacancies generated by a single damaging ion within the active volume

ii) Fully depletion conditions: $\frac{\partial F}{\partial V_{S}}=\frac{1}{d}$

iii) Constant carrier velocity profiles: $v_{n, p}(z)=v_{n, p} \Rightarrow \sigma_{n, p}^{e f f}=\left(\sigma_{n, p} \frac{v_{n, p}^{t h}}{v_{n, p}}\right)$

Under these conditions, the solution of eq. (119) is:

$$
C C E=\int_{0}^{d} d x \cdot \gamma(x)\left\{\frac{\lambda_{n}}{d}\left[1-\exp \left(-\frac{d-x}{\lambda_{n}}\right)\right]+\frac{\lambda_{p}}{d}\left[1-\exp \left(-\frac{x}{\lambda_{p}}\right)\right]\right\}
$$

which is the generalized Hecht's equation [63]. If the generation occurs at the cathode $(x=0)$, i.e.

$Y(x)=\delta(x)$, where $\delta(x)$ is the Dirac's delta function, the charge collected at the sensing electrode originates only from one carrier type (electrons) and eq. (123) converges to the conventional Hecht's equation. It is worth noticing that, in this particular case, the drift length is a decreasing function of the damaging ion beam fluence:

$$
\lambda_{n}=v_{n} \cdot\left(\frac{\tau_{n}^{0}}{1+\Phi \cdot k_{n} \cdot \frac{V_{T} \cdot \sigma_{n} v_{n}^{t h}}{d} \cdot \tau_{n}^{0}}\right)
$$

\section{Case II: low level of damage.}

In this case, we assume a low level of damage in the pristine ideal junction and the drift length being much longer than the extent of the depletion region (i.e $\lambda_{n, p}>\lambda_{n, p}^{0}>>$ d). Under these approximations, the exponentials in eq. (119) can be expanded in Taylor series, providing the following approximated expression:

$$
\begin{aligned}
& C C E \cong \int_{0}^{d} d x \cdot \gamma(x)\left\{\int_{x}^{d} d y \cdot \frac{\partial \vec{F}(y)}{\partial V_{s}} \cdot \exp \left[-\int_{x}^{y} \frac{d z}{\lambda_{n}(z)}\right]+\int_{0}^{x} d y \cdot \frac{\partial \vec{F}(y)}{\partial V_{s}} \cdot \exp \left[-\int_{y}^{x} \frac{d z}{\lambda_{p}(z)}\right]\right\}= \\
& =1-\Phi \int_{0}^{d} d z V(z) \cdot\left\{k_{n} \cdot \sigma_{n}^{e f f}(z) \cdot \int_{z}^{d} d y \cdot \frac{\partial \vec{F}(y)}{\partial V_{s}} \cdot \int_{0}^{z} d x \cdot \gamma(x)+k_{p} \cdot \sigma_{p}^{e f f}(z) \cdot \int_{0}^{z} d y \cdot \frac{\partial \vec{F}(y)}{\partial V_{s}} \cdot \int_{z}^{d} d x \cdot \gamma(x)\right\}
\end{aligned}
$$

By definition:

$$
\int_{0}^{d} d x \cdot \gamma(x)=\int_{0}^{d} d y \frac{\partial \vec{F}(y)}{\partial V_{s}}
$$

Eq. (125) provides the evidence for different roles played by the two carriers. To simplify, let us consider a vacancy profile localized at $x=x_{0}$, i.e. $V(z)=V_{0} \cdot \delta\left(z-x_{0}\right)$. If the generation profile extinguishes at a depth $x<x 0$, i.e. $y(x)=\theta\left(x_{0}-x\right)$, where $\theta$ is the Heaviside step function, it is apparent that only electrons, travelling from 0 to $d$, will cross the damaged region and might suffer

recombination, whereas the contribution of holes, moving in the opposite direction, is null. This simple consideration provides the basis of the experimental protocol, used for 
the measurements provided below as examples, and allows the recombination/trapping of both types of charge carriers (electrons and holes), that contribute to the CCE degradation, to be discriminated.

\section{Case III: derivation of the NIEL displacement damage formula}

Starting from eq.(125), and assuming the constant vacancy profile up to a depth $\mathrm{R}<\mathrm{d}$ (i.e $\left.V(z)=\theta(R-z) \cdot \frac{V_{T}}{R}\right)$ the linear degradation of CCE can be expressed as follows:

$C C E \cong 1-\Phi \frac{V_{T}}{R} \int_{0}^{R} d z \cdot\left\{k_{n} \cdot \sigma_{n}^{e f f}(z) \cdot \int_{z}^{d} d y \cdot \frac{\partial \vec{F}(y)}{\partial V_{s}} \cdot \int_{0}^{z} d x \cdot \gamma(x)+k_{p} \cdot \sigma_{p}^{e f f}(z) \cdot \int_{0}^{z} d y \cdot \frac{\partial \vec{F}(y)}{\partial V_{s}} \cdot \int_{z}^{d} d x \cdot \gamma(x)\right\}$

This expression can suitably be connected to the phenomenological concept of "displacement damage dose" $\mathrm{D}_{\mathrm{d}}$ proposed by Messenger et al. [24], which is the displacement damage energy deposition per unit mass of material calculated as the product of the particle fluence and the respective non-ionizing energy loss (NIEL), which is an estimate of the rate of energy loss due to atomic displacements as a particle traverses a material. The CCE degradation is expressed in term of $D_{d}$ through the following expression:

$$
\mathrm{CCE}=1-\mathrm{K} \text { ed } \cdot \mathrm{Dd}
$$

where $K_{e d}$ is the equivalent damage factor and the displacement damage dose $D_{d}$ is defined as follows:

$$
D_{d}=\left(\frac{M}{\rho} \cdot \frac{V_{T} \cdot \Phi}{R}\right)
$$

where $M$ is the average energy threshold value deposited in an ion-atom collision for the generation of a single vacancy $(M=54.5 \mathrm{eV}$ in Si) and $\rho$ is the mass density of the irradiated material (for $\mathrm{Si}, \rho=2.32 \mathrm{~g} \cdot \mathrm{cm}^{-3}$ ) [74]. The constant terms in the round brackets in eq. (129) is named "adjusted NIEL" and represents the rate at which the energy is lost to nonionizing events per unit length normalized for the material density, so that the units are typically $\mathrm{eV} \cdot \mathrm{cm}^{2} \cdot \mathrm{g}^{-1}$.

By comparing eqs. 127-129, we obtain the following analytical expression for the equivalent damage factor:

$K_{e d}=\frac{\rho}{M} \int_{0}^{R} d z \cdot\left\{k_{n} \cdot \sigma_{n}^{e f f}(z) \cdot \int_{z}^{d} d y \cdot \frac{\vec{F}(y)}{\partial V_{s}} \cdot \int_{0}^{z} d x \cdot \gamma(x)+k_{p} \cdot \sigma_{p}^{e f f}(z) \cdot \int_{0}^{z} d y \cdot \frac{\partial \vec{F}(y)}{\partial V_{s}} \cdot \int_{z}^{d} d x \cdot \gamma(x)\right\}$

This expression explicitly shows the dependence of the equivalent damage factor $\mathrm{K}_{\mathrm{ed}}$ on: i) the electrostatics of the device, ii) the carrier transport and recombination features

and iii) the ion probe ionization profiles, summarized by the terms $\left(k_{n, p} \cdot \sigma_{n, p}^{e f f}\right)$, $\frac{\partial F}{\partial V_{s}}$ and $\mathrm{Y}$, respectively. If the experimental conditions (i.e. device polarization and ion probe) are maintained constant as well as the ranges of the damaging ions, the NIEL approach can effectively correlate the CCE degradation of an electronic device induced by different radiation sources or energies, as clearly demonstrated in [31,23]. If one or more of these conditions are not fulfilled, the complete expression given by eq. 119 has to be used.

\section{Example: Cluster Defects in Silicon}

A depth profiling of partly damaged silicon layer implanted with scanned 8.3 MeV Si ion micro- beam has been performed by means of DLTS measurements at two different voltage settings [75], in order to distinguish defects which are created in the vacancyrich and interstitial-rich regions. DLTS analysis of the vacancy-rich region in self- 
implanted silicon reveals a formation of the vacancy-related defect(s) with activation energy at Ec-0.4 eV. Direct measurement of the electron capture kinetics associated with these states, prior to any annealing; do not show the exponential behaviour typical for simple point defects. The logarithmic capture kinetics is in accordance with the theory of majority carrier capture at extended or cluster-related defects [76].

Results of DLTS spectroscopy performed on implanted silicon diodes are shown in Fig. 50, for 8.3 MeV Si single ion implantation and 0.8 MeV neutron irradiation. The depth profiling of the vacancy-rich surface side of the implant tail and interstitial-rich region near the implant peak spectra are shown as well. The section of DLTS spectra corresponding to E1trap formed in the implant tail as a function of the filling time $\left(t_{p}\right)$, and the results of carrier capture cross-section analysis of the E1 trap showing logarithmic behaviour, typical for complex (cluster) defect structures, is also shown in the figure. The inset in fig.50 (d) shows the exponential dependence of carrier capture cross section typical for point defects.

These DLTS results suggest that small clusters of $\mathrm{V}_{2}(-/ 0)$ defects with activation energy close to $\mathrm{E}_{\mathrm{C}}-0.4 \mathrm{eV}$ are created in the highly disordered regions of silicon before any annealing [35]. Complete suppression of the $\mathrm{VO}$ and $\mathrm{V}_{2}(=/-)$ states in DLTS results is interpreted according to the model of local compensation of the carrier concentration in highly disordered regions located within the ion cascade region.

DLTS spectra of phosphorus-doped CZ-grown silicon irradiated with fast neutrons $(0.7$ $\mathrm{MeV}$ ) and 6.5 MeV O, 10.5 MeV Si, 10.5 MeV Ge, 11 MeV Er ions are shown in Fig.51. The spectra have been vertically displaced for clarity. All measurements are recorded upon irradiation and no annealing has been carried out. The neutron flux was chosen based on the simulation results, in order to introduce the same amount of the vacancyrelated defects (compared to Si ion implantation). Two deep traps with their DLTS peak maxima at about 92 and $188 \mathrm{~K}$ for an emission rate window of $4.65 \mathrm{~s}-1$ are observed in the spectrum of the fast neutron irradiated sample.

One prominent defect state corresponding to DLTS maximum at around $196 \mathrm{~K}$ has been observed in all ion-implanted samples. Arrhenius plots of $\mathrm{T}^{2}$-corrected emission rates for all observed traps which act as electron traps are shown in Fig.52. The derived values for the activation energy for electron emission $\left(\Delta \mathrm{E}_{\mathrm{ne}}\right)$, the pre-exponential factor $\left(A_{n e}\right)$ and the capture cross section, together with the defect identification, are given in the Table 4. The calculated energy levels of traps observed in the neutronirradiated sample are in agreement with well-established values in the literature $[77,78]$. Therefore those traps have been assigned to the $\mathrm{VO}(-/ 0)$ and $\mathrm{V}_{2}(-/ 0)$ defects. For the low phosphorous-doped silicon wafers, as the one used in our study with $[\mathrm{P}]$ of the order of $10^{14} \mathrm{~cm}^{-3}$, the formation of vacancy-phosphorus complex state (VP defect) can be neglected [79]. The DLTS peak related to the $\mathrm{V}_{2}(-/ 0)$ defect exhibits an asymmetric broadening, a feature which is usually associated with more complex defects [80]. Another interesting aspect of fast neutron irradiation, is the suppression of the DLTS peak related to the double acceptor state of divacancy $V_{2}(=/-)$. It is a well-known fact that for neutron irradiation [77] and ion implantation [81], the ratio between DLTS signals associated with two charge states of divacancy is changing, compared to the electron irradiation when the intensity of DLTS signals originated from both charge states is equal. Moreover, it is already reported by Vines et al. [82] that DLTS intensities of VO(10) and $\mathrm{V}_{2}(=/-)$ related traps are decreasing after He, C, Si and I implantations. In particular, the DLTS signal originating from $\mathrm{V}_{2}(=/-)$ defect is almost completely vanished after the I implantation, as the suppression is correlated with increasing ion mass.

It should be noted that the values obtained for the activation energies of the $V_{2}(-/ 0)$ defect observed in all irradiated samples (Table 4) are slightly lower than the benchmark 
value [83] in some cases, but within the range of reported experimental deviations. Moreover, similar values for the carrier capture cross section (at least the same order of magnitude) justify an assumption of the same defect state. Comparing results in Table 4 with the literature data, we have assigned the most dominant electron trap in all ion implanted samples to the single acceptor state of divacancy $\mathrm{V}_{2}(-/ 0)$. In our previous study [35], we have already observed a similar effect, the suppression of DLTS signals related to the $\mathrm{VO}(-/ 0)$ and $\mathrm{V}_{2}(=/-)$ defects upon single ion $8.3 \mathrm{MeV} \mathrm{Si}$ implantation. We have also shown that in the self-implanted silicon the prominent broad trap with the calculated activation energy for electron emission of $0.4 \mathrm{eV}$, and related to the $\mathrm{V}_{2}(-/ 0)$ defect, comes from the closely spaced singly charged divacancies which are formed directly from the nearest monovacancies originating from the single ion impact cascade [35]. These results agree with the model of local compensation of the carrier concentration in highly disordered regions located within the ion cascade region. Results presented in Fig.51 confirm our previous findings.

Moreover, two interesting features have been observed: (i) the shift of the $\mathrm{V}_{2}(-/ 0)$ related DLTS peak maxima to the higher temperatures (which subsequently leads to an increase of the activation energy, Table 4) with the increasing ion mass, and (ii) the appearance of the high temperature shoulder upon heavy ion implantations. The existence of the new deep defect state which captures an electron, evidenced as the high temperature shoulder of the prominent DLTS peak of the $\mathrm{V}_{2}(-/ 0)$ defect state, is observed upon $\mathrm{Ge}$ ion implantation, but it is more clearly visible upon $\mathrm{Er}$ ion implantation. However, we have not been able to reliably estimate values for the activation energy and capture cross section for this newly observed state. To our best knowledge, this defect has not yet been reported or identified in the literature yet. Further studies are needed to clarify the identity of these electron traps.

To further check the behaviour of the observed vacancy-related defects, a comparison was performed of the measured values of $\Delta E_{n e}$ and $A_{n e}$ for the $V_{2}(-/ 0)$ related defect, for the fast neutron irradiated sample and for all ion implanted samples. The MeyerNeldel rule connects the similar thermally activated processes, is used in distinguishing different types of defects. According to the Meyer-Neldel rule [84], the $\ln \left(A_{n e}\right)$ should be a linear function of $\mathrm{DE}_{\text {ne }}$ for the same type of defects. Comparing the measured values (Table 3) for the dominant defect in all samples, which we have assigned to the $\mathrm{V}_{2}(-/ 0)$, shows an excellent linear correlation, as shown in Fig.53. The fact that the values obtained upon neutron irradiation fit perfectly within results obtained upon ion implantation strongly supports the conclusion that the observed defects in all samples are indeed related to the $\mathrm{V}_{2}(-/ 0)$ defect [85]. As already mentioned, the activation energy for electron emission from the $\mathrm{V}_{2}(-$

10) defect is slightly increasing as the mass of the implanted ions is increasing. Heavy ions produce more complex cascade regions, more complex defects, which lead to the broadening of the DLTS spectra and therefore have an effect on the estimated activation energy. The ion mass effect on vacancy-related defects in the n-type $\mathrm{CZ}$ silicon implanted with heavy ions has been reported by Vines et al [82]. Together with a decrease of the intensity of DLTS peaks related to the $\mathrm{VO}$ and $\mathrm{V}_{2}(=/-)$ defects with increasing ion mass, the capture cross section measurements for the $V_{2}(-/ 0)$ defect have shown a change in the defect kinetics from the point-like to the extended defect as a function of the ion mass.

The single heavy ion implantation leads to the formation of dominant electron traps assigned to the single acceptor state of divacancy $\mathrm{V}_{2}(-/ 0)$. Broadening of the DLTS peak originating from $\mathrm{V}_{2}(-/ 0)$ defects formed in the single heavy ion implanted silicon is ascribed to divacancy perturbed by local environments of close lying point defects [85]. The complete suppression of DLTS signal originating from the $\mathrm{VO}(-/ 0)$ and $\mathrm{V}_{2}(=/-)$ defects in the low-doped n-type silicon implanted by single heavy ions can be explained by (i) the local depletion of the carrier concentration in the highly disordered regions, and (ii) the effect of the microprobe-assisted single ion implantation. 
The calculated activation energies for electron emission for the $\mathrm{V}_{2}(-/ 0)$ related traps observed upon MeV's implantation of $\mathrm{O}, \mathrm{Si}, \mathrm{Ge}$ and $\mathrm{Er}$ into Si follows the Meyer-Neldel rule. A slight increase of the activation energy for the electron emission for the $V_{2}(-/ 0)$ defect is correlated with increasing ion mass.

\section{Example: of Radiation Damage Effects on Electronic Properties of Silicon Capacitance Measurements}

lonizing radiation, particularly low-energy hydrogen, creates deep centers and influences the shallow donor concentration in n-type silicon [86]. Deep and shallow donors can be differentiated by their respective capacitance-voltage (C-V) measurements at different sample temperatures. At a given temperature, the maximum frequency that a center can respond to depends on its energy level. Therefore, the $\mathrm{C}-\mathrm{V}$ characteristics of irradiated photodiodes should ideally be measured as a function of the frequency in order to obtain the energy and depth distribution of ionized centers. The C$\mathrm{V}$ characteristics of irradiated photodiodes were measured at a constant frequency of 1 $\mathrm{kHz}$, which was low enough to be sensitive to some of the expected deep levels. In order to achieve a similar total energy deposition through NIEL for different ions, fluences of $2.45 \times 10^{11} \mathrm{~cm}^{-2}, 7.65 \times 10^{9} \mathrm{~cm}^{-2}$, and $1.4 \times 10^{9} \mathrm{~cm}^{-2}$ were used for protons, lithium, and oxygen, respectively. As can be seen in Fig.54, for lower bias voltages, the $\mathrm{C}-\mathrm{V}$ curve of the photodiode irradiated with protons over the whole sensitive region differs significantly from results obtained for the virgin and photodiodes irradiated with heavier ions due to ionization effects of proton induced defects within space charge region [30].

From the $\mathrm{C}-\mathrm{V}$ characteristics for the irradiated photodiodes, the resultant doping profiles were calculated. No significant observable changes in the doping concentration were observed for lithium- and oxygen-irradiated photodiodes at the aforementioned fluences. A high concentration of implanted protons (on the order of $10^{14} \mathrm{~cm}^{-3}$ ), compared to the non-irradiated silicon doping (on the order of $10^{13} \mathrm{~cm}^{-3}$ ), in a narrow layer corresponding to the end of the proton range changed the total donor concentration distribution significantly in the active region of the photodiode (Figure55) [30].

Previous DLTS studies of unannealed hydrogen-irradiated silicon $[86,53]$ showed that, in addition to pure radiation defect levels, hydrogen-related defect levels at $E_{C}-0.30$ $\mathrm{eV}, \mathrm{E}(0.30)$, were formed in the fluence range of $10^{10}$ to $10^{13} \mathrm{H}^{+} \mathrm{cm}^{-2}$. As was suggested in [86], the $E(0.30)$ trap is a hydrogen-related defect formed through following process: $(\mathrm{P}-\mathrm{V})+\mathrm{H} \rightarrow(\mathrm{H}-\mathrm{V})+\mathrm{P}$.

A mismatch between the positions of the isolated peak in the hydrogen irradiationrelated defect concentration distribution $\mathrm{N}_{T}$ and TRIM simulated peak of the implanted hydrogen ion distribution $\mathrm{H}^{+}$(Fig.56) might be explained by the radiation-simulated migration model purposed by Belykh et al. [87]. The features of the induced defect profile by high-energy ion irradiation are likely to be found in non-equilibrium local excitations introduced by fast ions into the electronic subsystem of a solid. The relaxation of electronic excitations on atoms located off lattice sites (interstitial atoms, atoms included into extensive defects) proceeds much more effectively than on atoms occupying regular positions. This may lead to a considerable increase in the mobility of defect forming atoms (due to their selective heating). This suggests that the defects produced at the end of range by preceding ions migrate to the surface under the relaxation triggered by electronic excitations introduced by subsequent fast ions. Apparently, the described mechanism is effective only in the case of the multiple overlappings of fastion track cross-sections. Irradiations preformed with the ion microprobe focused ion beams with current density of the order of $\mu \mathrm{A} / \mathrm{cm} 2$ or higher, fulfill that necessary 
condition and enable the radiation-stimulated migration of defects related to implanted hydrogen atoms from the bulk towards p+-n junction [30].

The C-V technique was also used as a follow-up study of spontaneous self-annealing at room temperature of the irradiated samples. By rescaling the $C-V$ data for the change in the built-in voltage due to the lifetime-relaxation type transition, the remaining capacitance changes could be interpreted as nuclear recoil-induced damage located at the fringe of the particle range. The calculated initial capacitance depth profile $\mathrm{C} 1$ obtained 2 weeks after the irradiation is used for the normalization of the capacitance depth profile values C2 and C3 obtained 6 weeks and 30 weeks after the irradiation, respectively. Normalized capacitances, as a function of depth for $430 \mathrm{keV}$ protons, are plotted in Fig.57. The black line represents the normalized initial value. Subsequent normalized capacitance values $\mathrm{C} 2 / \mathrm{C} 1$ and $\mathrm{C} 3 / \mathrm{C} 1$ are depicted with hollow circles and black circles, respectively. The calculated normalized capacitance peaks are positioned at a depth that is slightly larger than the end of the range for $430 \mathrm{keV}$ protons in silicon.

The sensitivity of the normalized capacitance to the evolution of electrically active defects created by particle irradiation is illustrated for low fluence irradiation by $6.5 \mathrm{MeV}$ oxygen ions (Fig.58). Here, data taken 6 and 31 weeks after irradiation were normalized. Similar behavior of the normalized capacitance was observed for the photodiode irradiated by a low fluence of $2.15 \mathrm{MeV}$ lithium ions. From Figs. $\mathbf{5 7}$ and $\mathbf{5 8}$, it can be seen that the rate of the room temperature spontaneous self-annealing process of the sample irradiated by protons is larger compared to the sample irradiated by oxygen ions. Over the course of 28 weeks, the maximum value of the normalized capacitance across the potential barrier of the sample irradiated by protons decreased by approximately $30 \%$, while the sample irradiated by oxygen and lithium ions decreased approximately $4 \%$ and $10 \%$, respectively [30]. This result may be attributed to the lower thermal stability of hydrogen-induced complexes compared to that of implanted oxygen (and lithium) - induced complexes at RT. 


\section{Leakage Current Measurements}

Energetic particle radiation creates lattice damage in solids, and in semiconductor junctions, this translates in the formation of additional energy levels in the forbidden energy gap and an increase in the leakage current. Since the reverse leakage current of advanced silicon photodiodes used in nuclear radiation detection is low, measurement of changes in leakage current can be a very sensitive tool for monitoring ion implantation doses [88] and the induced radiation damage in a diode. The reverse leakage current in semiconductor diodes consists of generation and diffusion components. The generation current originates from carriers thermally generated in the depletion region, while the diffusion current originates from inward diffusing carriers generated outside the depleted region. By measuring the I-V characteristics at voltages much less than the thermal voltage $\mathrm{kT} / \mathrm{q}$, one can easily separate the total diffusion current. After subtracting the diffusion current, the generation current component is all that remains.

The I-V characteristics of photodiodes irradiated by $5.5 \mathrm{MeV}$ alpha particles were measured to investigate the dependence of the generation current on ion fluence. An almost exact linear relationship was obtained for the fluence dependence of the generation current in the entire fluence range (Fig. 59) [30], which is in agreement with previous similar studies [88].

A similar procedure was conducted for $430 \mathrm{keV}$ protons, $2.15 \mathrm{MeV}$ lithium, and $6.5 \mathrm{MeV}$ oxygen ions irradiation. The measured leakage currents in photodiodes irradiated by energetic ions were scaled to a displacement damage dose, $D_{d}=3.9 \times 10^{11} \mathrm{MeV} / \mathrm{g}$, of protons in order to obtain a correlation between different types of ionizing radiation, as is shown in Fig.60.

At a reverse bias of $100 \mathrm{~V}$, an increase in the leakage current from approximately 2.3 $\mathrm{nA} / \mathrm{cm}^{2}$ for the unirradiated sample, to $1.7 \mu \mathrm{A} / \mathrm{cm}^{2}, 2.4 \mu \mathrm{A} / \mathrm{cm}^{2}$, and $3 \mu \mathrm{A} / \mathrm{cm}^{2}$ for samples irradiated with protons, lithium, and oxygen ions, respectively, was measured. Despite data scaling as per the

previously mentioned procedure, differences in the leakage currents as a function of applied bias voltage were observed, which possibly indicate the number of electrically active centers is not equal to the number of primarily displaced atoms from lattice sites. From this result, it appears that for the same number of created vacancies, heavier ions produce more active recombination centers. From Fig.60, it can be observed that the leakage current for the most frequently used bias voltage ranges in ion micro-beam applications (zero up to breakdown voltage) for a photodiode irradiated by oxygen ions is larger than the leakage current of a photodiode irradiated by protons of the same displacement damage. It is possible is that excess hydrogen and /or lithium compensates for part of the electrically active centers [89].

\section{Final Remarks}

The nuclear techniques reviewed here can offer major benefits in advancing the technology of semiconductors and oxide semiconductors (OS), from sample preparation through to the sample characterisation. They are adequate for OS requirements of surface and near surface modification and characterisation, and can be beneficial in tailoring their properties bringing these materials closer to industrial applications.

The application of nuclear techniques requires the building and maintenance of major facilities, and therefore their cost can be high. In some cases, the need of high energies requires the use of accelerators or the use of radioactive sources. In addition the use of 
nuclear techniques and facilities require specific expertise, and are subject to strict safety regulations, and these are the main reasons why these techniques are mainly concentrated at national nuclear facilities.

The nuclear techniques are frequently associated with the use of isotopes which are required in order to distinguish the tracer particle from the background formed by the same elements. 


\section{References}

[1] J Nowotny, Oxide Semiconductors for Solar Energy Conversion, CRC, Boca Raton, 2012

[2] G. Dearnaley, J. H. Freeman, R. S. Nelson, J. Stephen, Ion Implantation, American Elsevier Publishing Co. New York, (1973)

[3] S Azimi, J Song, Z Y Dang, H D Liang and M B H Breese, J. Micromech. Microeng. 22, 113001, (2012)

[4] A. Markwitz, D.A. Carder, T. Hopf, J. Kennedy, T.K. Chan, A. Mucklich, T. Osipowicz, , NIM B 373, 199, (2012)

[5] H. Yamashita, M. Harada, J. Misaka, M. Takeuchi, Y. Ichihashi, F. Goto, M. Ishida, T. Sasaki, M. Anpo, J. Synchrotron Rad. 8, 569, (2001)

[6] M. Takeuchi, M. Matsuoka, M. Anpo, Res. Chem. Intermed 38, 1261, (2012)

[7] M. Ni, M. K. H. Leung, D. Y. C. Leung, K. Sumathy, Renewable and Sustainable Energy Rev. 11, 401, (2007)

[8] A. L. Stepanov, Rev. Adv. Mat. Sci. 30, 150, (2012)

[9] E. Alves, N. Franco, N. P. Barradas, B. Nunes, J. Lopes, A. Cavaleiro, M. Torrell, L. Cunha, F. Vaz, NIM B 272, 61, (1012)

[10] C-L. Jia, Z-N. Wei, R. Zhou, NIM B 313, 50,

(2013) [11] J. F. Ziegler, NIM B 219-220, 1027,

(2004)

[12] J.R. Tesmer, M. Nastasi, 83, 'Handbook of Modern Ion Beam Materials Analysis', MRS, Pittsburgh (1995)

[13] M.B.H. Breese, E.J. Teo and L. Huang, NIM B 260, 288, (2007)

[14] L. C. Fledman, J. W. Mayer, S. T. Picraux, 'Materials Analysis', Accademic Press NY, (1985)

[15] D. D. Cohen, E. Clayton, Academic Press, Sydney, Ch 5, (1989)

[16] A. Uedono, S. Ishibashi, T. Ohdaira, R. Suzuki, J. Cryst. Growth 311, 3075, (2009)

[17] T. Yamaki, T. Umebayashi, T. Sumita, S. Yamamoto, M. Maekawa, A.

Kawasuso, H. Itoh, NIM B 206, 254, (2003)

[18] M. Mayer, Report IPP 9/113, Max-Plank Institut fur Plasmaphysik, Garching,

Germany 1997 [19] M.B.H. Breese, J. Appl. Phys. 74(6) (1993) 3789-3799

[20] J.F. Ziegler, J.B. Biersack, U.Littmark, The Stooping and Range of lons in Solids, Pergamon, New York, 1985.

[21] [G.P. Summers, E.A. Burke, C.J. Dale, E.A. Wolicki, P.W. Marshall, and M.A. Gehlhausen, IEEE TNS, NS-34, 1134 (1987)

[22] A.B. Cambell, A.R. Knudson, W.J. Stapor, G.P. Summers, M.A. Xapsos, M. Jessee, T. Palmer, R. Zuleeg, and C.J. Dale, Particle damage effects in GaAs JFET test structures, IEEE TNS, NS-33, 1435 (1986).

[23] G.P. Summers, E.A. Burke, P. Shapiro, S.R. Messenger, and R.J. Walters, IEEE TNS, NS-40, 1372 (1993)

[24] S.R. Messenger, E.A. Burke, G.P. Summers, M.A. Xapsos, R.J. Walters, E.M. Jackson, B.D. Weaver, IEEE TNS NS-46, No. 6, Dec 1999, 1595 - 1602

[25] M. Huhtinen, Nucl. Instr. Meth. Phys. Res. A 491 (2002) 194-215 
[26] S.M. Sze, Physics of Semiconductor Devices, 2nd Edition, Wiley, New York 1981

[27] E. Chason, S.T. Picraux, J.M. Poate, J.O. Borland, M.I. Current, T. Diaz de la Rubia, D.J. Eaglesham, O.W. Holland, M.E. Law, C.W. Magee, J.W. Mayer, J. Melngailis, and A.F. Tasch, Applied physics review, J. Appl. Phys. 81 (1997) 6513.

[28] L. Pelaz, L.A. Marques, M. Aboy, P. Lopez, and I. Santoz, Eur. Phys. J. B72 (2009) 323.

[29] C. Leroy and P.G. Ranciota, Rep. Prog. Phys. 70 (2007) 493.

[30] Z. Pastuovic, M. Jaksic, G. Kalinka, M. Novak, A. Simon, IEEE Trans. Nucl. Sci. NS-56, (2009) 2457.

[31] Z. Pastuovic, E. Vittone, I. Capan, M. Jaksic, Appl. Phys. Lett. 98, (2011) 092101.

[32] I. Zamboni, Z. Pastuovic, and M. Jaksic, Diam. Relat. Mater. 31, (2013) 65.

[33] N. Iwamoto, B.C. Johnson, N. Hoshino, M. Ito, H. Tsuchida, K. Kojima and T. Ohshima, J. Appl. Phys. 113 (2013) 143714.

[34] T. Ohshima, S. Sato, M. Imaizumi, T. Nakamura, T. Sugaya, K. Matsubara, S. Niki, Solar Energy Materials \& Solar Cells 108, (2013) 263.

[35] Z. Pastuovic, I. Capan, R. Siegele, R. Jacimovic, J. Forneris, D.D. Cohen and E. Vittone, Nucl. Instr. Meth. Phys. Res. B 332, (2014) 298.

[36] N. Dharmarasu, M. Yamaguchi, J.C. Bourgoin, T. Takamoto, T. Ohshima, H. Itoh, M. Imaizumi and S. Matsuda, Majority- and minority-carrier deep traps in protonirradiated InGaAs space solar cells, Appl. Phys. Lett. 81(1), (2002) 64.

[37] K.K. Lee, T. Ohshima, A. Saint, T. Kamiya, D.N. Jamieson, H. Itoh, Nucl. Instr. Methods Phys. Res. B 210 (2003) 489

[38] [38] F. Nava, E. Vittone, P. Vanni, G. Verzellesi, P.G. Fuochi, C. Lanzierif, M. Glaser, Nucl. Instr. Meth. Phys. Res. A 505, (2003) 645.

[39] A. Castaldini, A. Cavallini, L. Rigutti, F. Nava, S. Ferrero and F. Giorgis, J. Appl. Phys. 98, (2005) 053706.

[40] F. Nava, A. Castaldini, A. Cavallini, P. Errani and V. Cindro, IEEE TNS NS-53 (5), (2006) 2977.

[41] F. Roccaforte, S. Libertino, V. Ranieri, A. Ruggiero, V. Massimino and L. Calcagno, J. Appl. Phys. 99, (2006) 013515.

[42] V.A.J. van Lint, T.M. Flanagan, R.E. Leadon, J.A. Naber, V.C. Rogers: Mechanism of radiation effects in electronic materials, John Wiley \& Sons, 1990

[43] Y. Shi, D.X. Shen, F.M. Wu, K.J. Cheng, J. Appl. Phys. 67 (1990) 1116

[44] B.R. Gossick, J. Appl. Phys. 30 (1959) 1214

[45] M.A. Green, J. Appl. Phys. 67 (1990) 2944

[46] G.Watkins, Gordon and Breach Science Publishers S.A., Yverdon ( $\mathrm{CH})$, 2nd edition, 1992 [47] A.O. Evwaraye, J. Appl. Phys. 47(9) (1976) 3776-3780

[48] A. Hallen, N. Keskitalo, F. Masszi, V. Nagl, J. App. Phys. 79 (1996) 3906-3914

[49] B.G. Svensson, J.L. Lindstrom, Phys. Rev. B 34(12) (1986) 8709-8717

[50] L.C. Kimerling, H.M. DeAngelis, J.W. Diebold, Solid State Communications 16 (1975) 171- 174

[51] S.D. Brotherton, P. Bradley, J. Appl. Phys. 53(8) (1982) 5720-5732 
[52] R. Nipoti, C. Donolato, D. Govoni, P. Rossi, G.P. Egeni, V. Rudello, Nucl. Instr. Meth. Phys. Res. B 136-138 (1998) 1340-1344

[53] J.F. Barbot, E. Ntsoenzok, C. Blanchard, J. Vernois, D.B. Isabelle, Nucl. Instr. Meth. Phys.

Res. B 95 (1995) $213-218$

[54] D.V. Lang, J. Appl. Phys. 45 (1974) 3023.

[55] J. L. Benton, J. Cryst. Growth 106 (1990) 116

[56] Y. Zohta, M.O. Watanabe, J. Appl. Phys. 53 (1981) 1809.

[57] L. Dobaczewski, A.R. Peaker, K. Bonde Nielsen, J. Appl. Phys. 96 (2004) 4689.

[58] S. Onoda, T. Hirao, J.S. Laird, H. Mori, H. Itoh, T. Wakasa, T. Okamoto, Y. Koizumi, Nucl. Instr. Meth. Phys. Res. B 206 (2003) 444-447

[59] S. Onoda, T. Hirao, J.S. Laird, H. Mori, H. Itoh, T. Wakasa, T. Okamoto, Y. Koizumi, Nucl. Instr. Meth. Phys. Res. B 210 (2003) 232-236

[60] A. Simon, G. Kalinka, M. Jakšić, Ž. Pastuović, M. Novak, A.Z. Kiss, Nucl. Instr. Meth. Phys. Res. B 260 (2007) 304-308

[61] F. Fizzoti, E. Colombo, A. Lo Giudice, C. Manfredotti, Z. Medunić, M. Jakšić, E. Vittone, Nucl. Instr. Meth. Phys. Res. B 260 (2007) 259-263

[62] E. Vittone, Z. Pastuovic, M.B.H. Breese, J. Garcia Lopez, M. Jaksic, J. Raisanen, R. Siegele,

A. Simon, G. Vizkelethy, Nucl. Instr. Meth. Phys. Res. B xxx (2016) xxx (accepted Dec2015). http://dx.doi.org/10.1016/j.nimb.2016.01.030.

[63] E. Vittone, F. Fizzotti, A. LoGiudice, C. Paolini, C. Manfredotti, Nucl. Instr. Meth. Phys. Res. B 161-163 (1999) 446-451

[64] E. Vittone, Nucl. Instr. Meth. Phys. Res. B 219-220 (2004) 1043-1050

[65] J.B. Gunn, Solid State Electronics 7 (1964) 739-742

[66] L. Grassi, J. Forneris, D. Torresi, L. Acosta, A. Di Pietro, P. Figuera, Nucl. Instr. Meth. Phys. Res. A 767 (2014) 99.

[67] T.H. Prettyman, Nucl. Instr. Meth. Phys. Res. A 422 (1999) 232-237

[68] W. Shockley, W.T. Read, Phys. Rev. 87 (1952) 835

[69] M.T. Robinson, I.M. Torrens, Phys. Rev. B 9 (1974) 5008.

[70] M.B.H. Breese, E. Vittone, G. Vizkelethy, P.J. Sellin, Nucl. Instr. Meth. Phys. Res. B 264 (2007) 345

[71] M. Martini, T.W. Raudorf, W.R. Scott, J.C. Waddington, IEEE TNS, NS-22 (1975) 145.

[72] W. Shockley, J. Appl. Phys. 9 (1938) 635

[73] E. Amaldi, O. D'Agostino, E. Fermi, B. Pontecorvo, F. Rasetti, E. Segre, Artificial radioactivity produced by neutron bombardment - II, Proc. R. Soc. Lond. A. Math. Phys. Sci. A 149 (1935) 522.

[74] S.R Messenger, E.A. Burke, G.P. Summers, R.J. Walters, IEEE TNS NS-51 No.6, Dec 2004, 3201-3206

[75] P. Leveque, H. Kortegaard Nielsen, P. Pellegrino, A. Hallen, B.G. Svensson, A. Yu. Kuznetsov, J. Wong-Leung, C. Jagadish, and V. Privitera, J. Appl. Phys. 93, 871 (2003)

[76] O. Yastrubchak, T. Wosinski, A. Makosa, T. Figielski, and A.L. Toth, Physica B 308-310 
(2001) 757. 
[77] I. Kovačević, V.P. Markevich, I.D.

Hawkins, B. Pivac and A.R. Peaker, J.

Phys. Condens. Matter 17 (2005)

S2229.

[78] R.M. Fleming, C.H. Seager, D.V. Lang,

E. Bilejec, J.M. Campbell, J. Appl. Phys.

104 (2008) 083702.

[79] G.D. Watkins, J.W. Corbett, Phys. Rev. 134 (1964) A1359.

[80] I. Capan, V. Borjanović, and B. Pivac, Solar Energy Materials and Solar Cells 91 (2007) 931.

[81] E.V. Monakhov, J. Wong-Leung, A. Yu.

Kuznetsov, C. Jagadish, and B.G. Svensson,

Phys.

Rev. B 65 (2002) 245201

[82] L. Vines, E.V. Monakhov, J. Jensen, A. Yu.

Kuznetsov, and B.G. Svensson, Phys. Rev. B 79 (2009) 075206.

[83] B.G. Svensson, C. Jagadish, A. Hallen, J. Lalita, Phys. Rev. B 55(16) (1997) 10498-10507

[84] V.P. Markevich, I.D. Hawkins, A.R.

Peaker, K.V. Emtsev, V.V. Emtsev, V.V.

Litvinov, L.I. Murin, L. Dobaczewski,

Phys. Rev. B 70 (2004) 235213.

[85] I. Capan, Z. Pastuovic, R. Siegele, R. Jacimovic, Nucl. Instr. Meth. Phys. Res.

B xxx (2016) xxx. http://dx.doi.

org/10.1016/j.nimb.2015.12.039

[86] L. Palmershofer and J. Reisinger, J. Appl. Phys. 72 (6), 1992, 2167-2173

[87] T.A. Belykh, A.L. Gorodishchensky, L.A. Kazak, V.E. Semyannikov, A.R. Urmanov, Nucl.

Instr. Meth. Phys. Res. B 51 (1990) 242-246

[88] P. Hazdra, V. Hašlar, J. Vobecky, Nucl. Instr. Meth. Phys. Res. B 96 (1995) 104

[89] R. Wunstorf: Systematische Untersuchungen zur Strahlenresistenz von Silizium-Detektoren fuer die Vervendung in HochenergiephysikExperimenten, PhD Dissertation, Universitaet Hamburg, Germany (1992) 


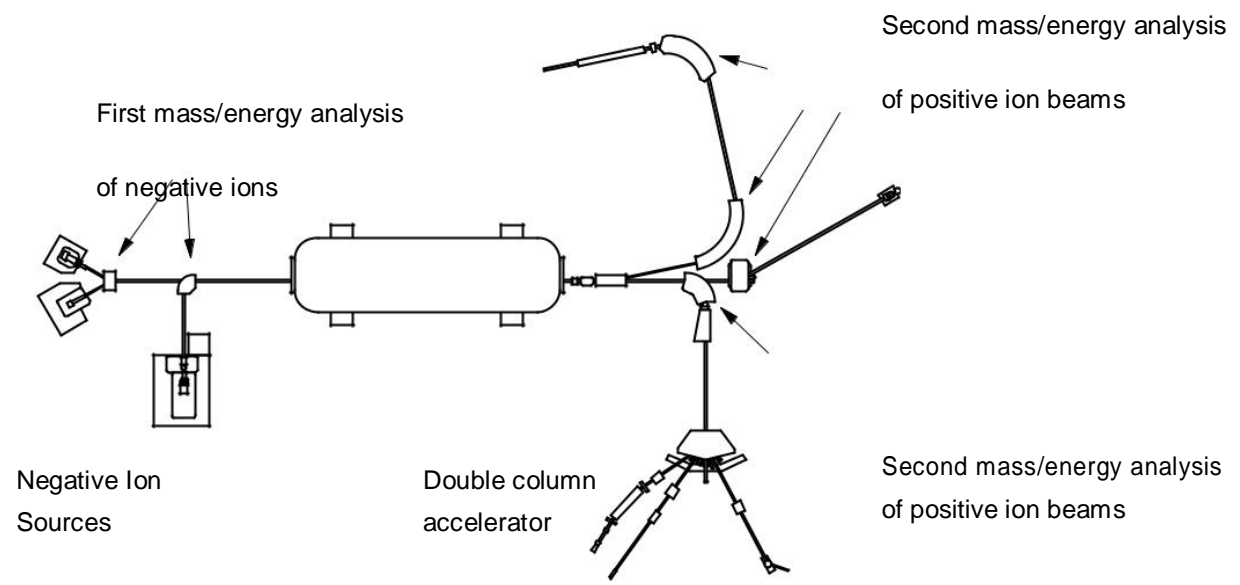

Experimental beamlines

Fig. 1: Schematic representation of an accelerator system, containing the ion sources, accelerator columns and experimental beamlines.

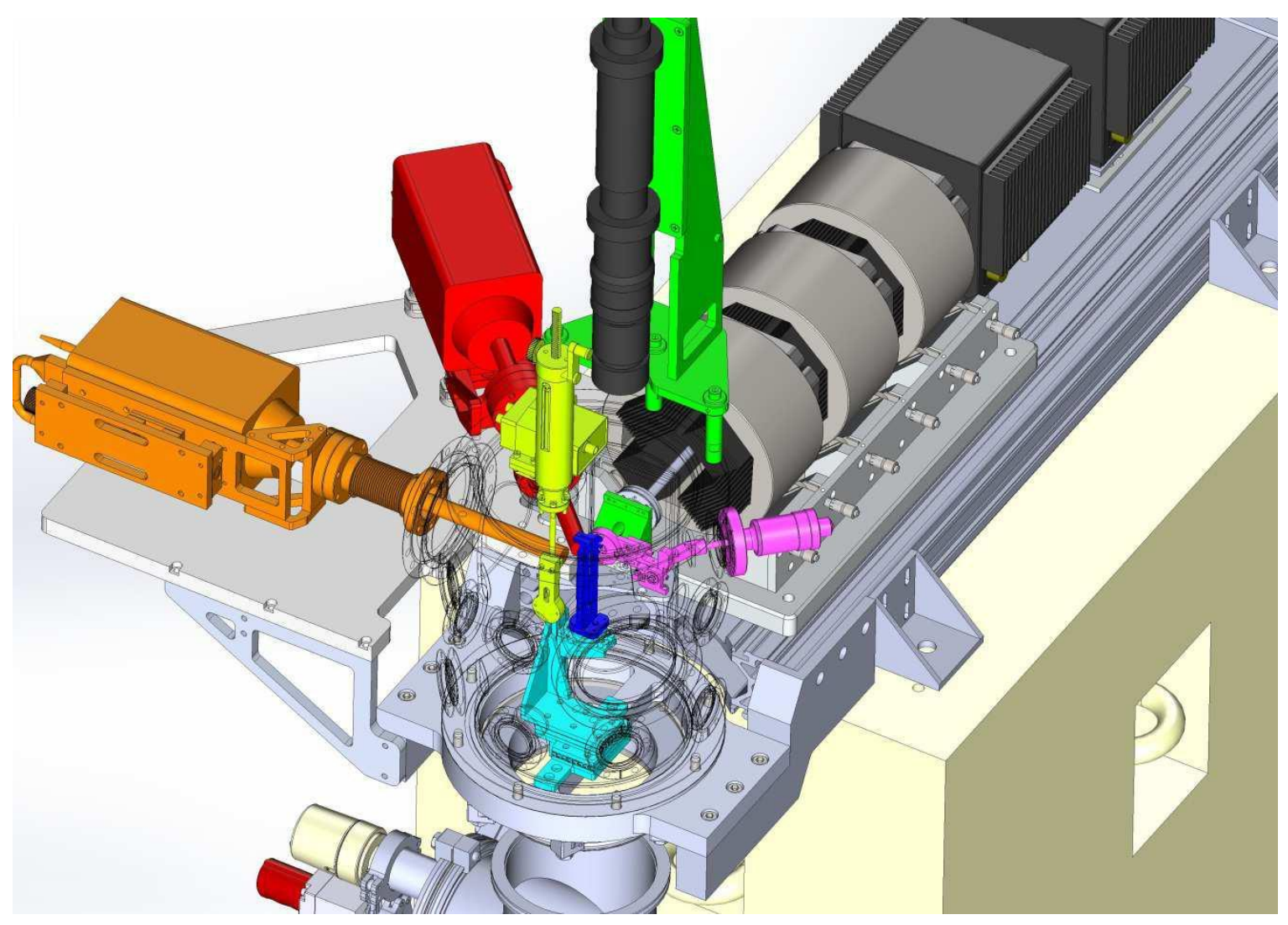


Fig. 2: Typical ion microprobe end-station schematics 


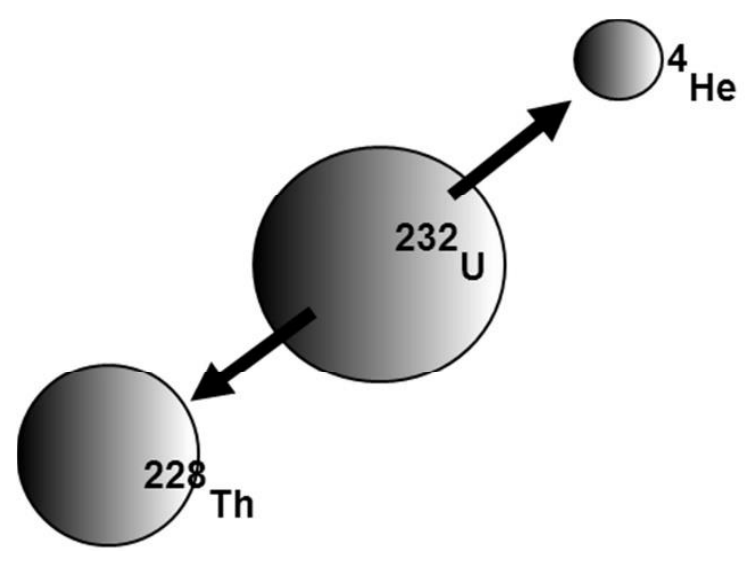

Fig. 3: Alpha decay example of ${ }^{232} \mathrm{U}$ to ${ }^{228}$ Th
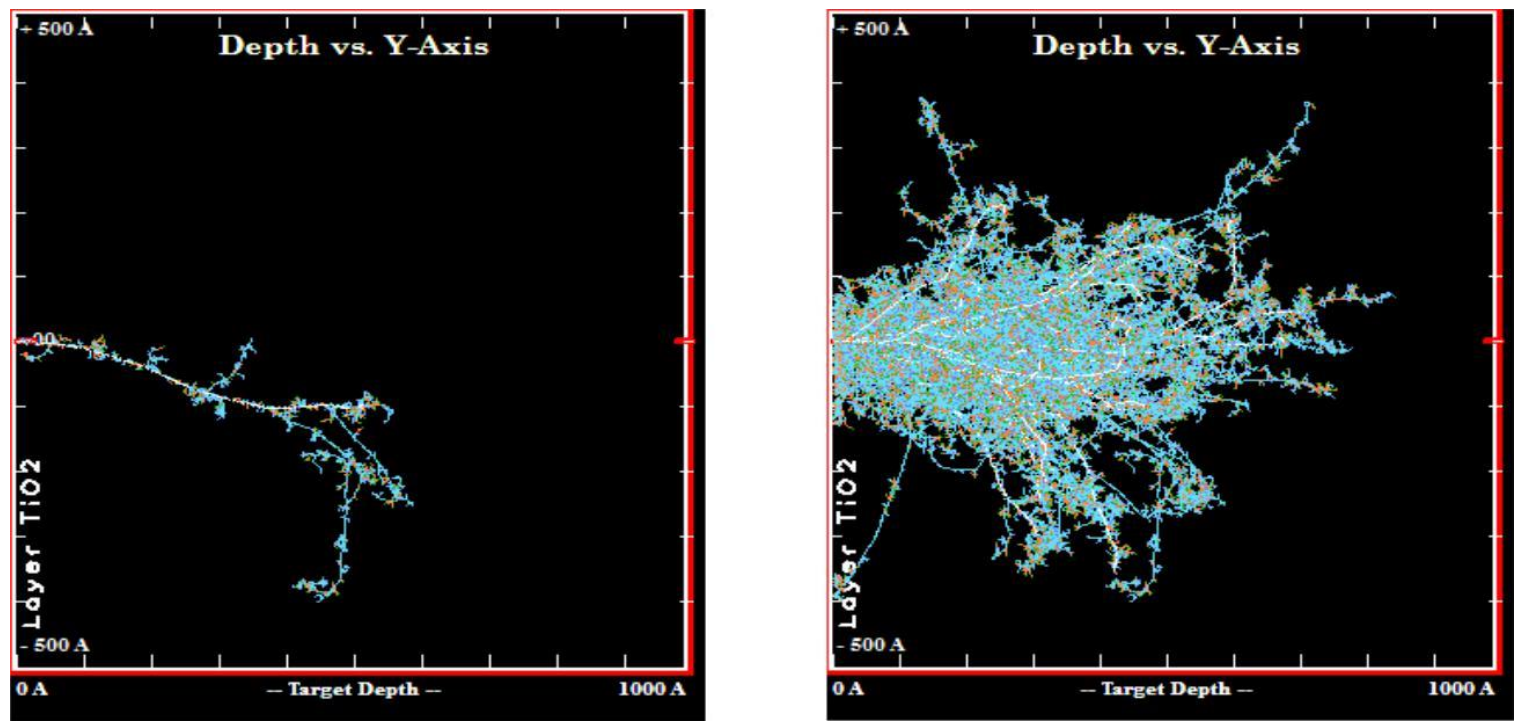

(a)

(b)

Fig. 4: Examples of damage accumulation track from a single $\mathrm{Ti}^{+1}$ ion of $50 \mathrm{keV}$ entering the surface of $\mathrm{TiO}_{2}(\mathrm{a})$, and the subsequent damage cascade of multiple similar tracks (b)

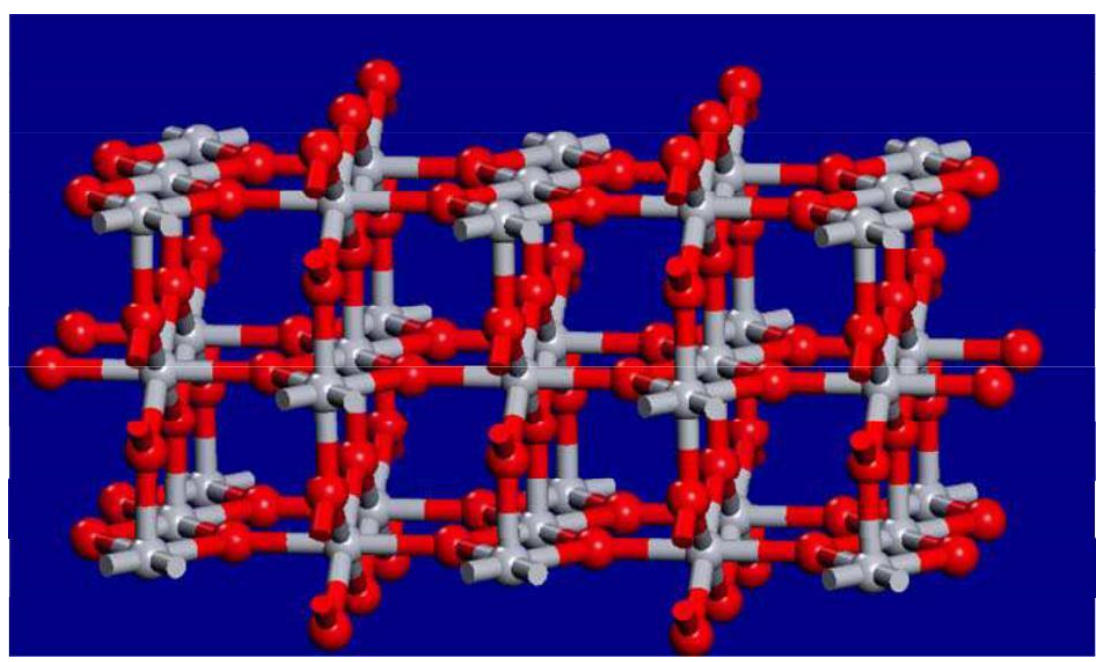


Fig. 5: Schematic representation of defect-free $\mathrm{TiO}_{2}$ (110) surface

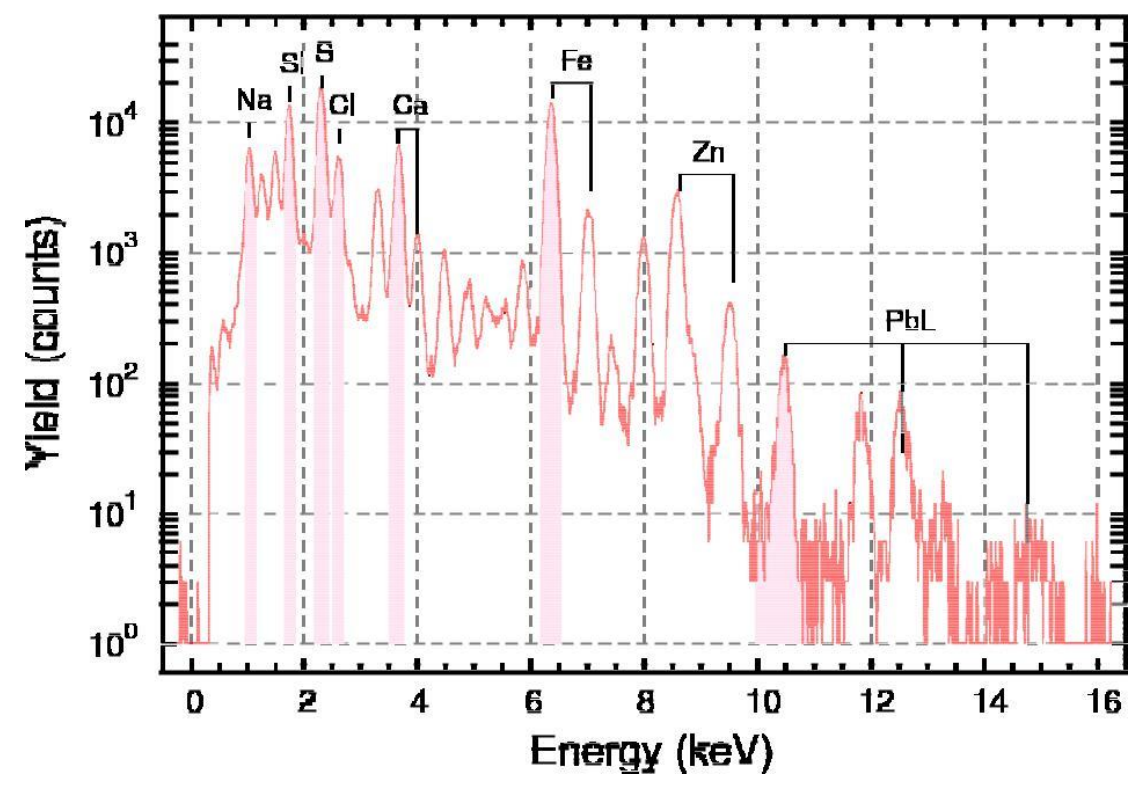

Fig. 6: PIXE spectrum of glass obtained with 2.5MeV protons

Table 1: The decay chain of ${ }^{232} \mathrm{U}$ to ${ }^{208} \mathrm{~Pb}$

\begin{tabular}{|l|l|l|l|l|l|l|l|l|}
\hline $232 \mathrm{U} \rightarrow$ & $228 \mathrm{Th}$ & $224 \mathrm{Ra}$ & ${ }_{220} \mathrm{Rn}$ & $216 \mathrm{Po}$ & $212 \mathrm{~Pb}$ & $212 \mathrm{Bi}$ & $208 \mathrm{TI}$ & $208 \mathrm{~Pb}$ \\
\hline$\alpha$ & $\alpha$ & $\alpha$ & $\alpha$ & $\alpha$ & $\beta$ & $\alpha$ & $\beta$ & \\
\hline 72 years & $\begin{array}{l}1.9 \\
\text { years }\end{array}$ & 3.6 days & 55 secs & $\begin{array}{l}0.15 \\
\text { secs }\end{array}$ & 10.64 hrs & 61 secs & 3 mins & stable \\
\hline
\end{tabular}




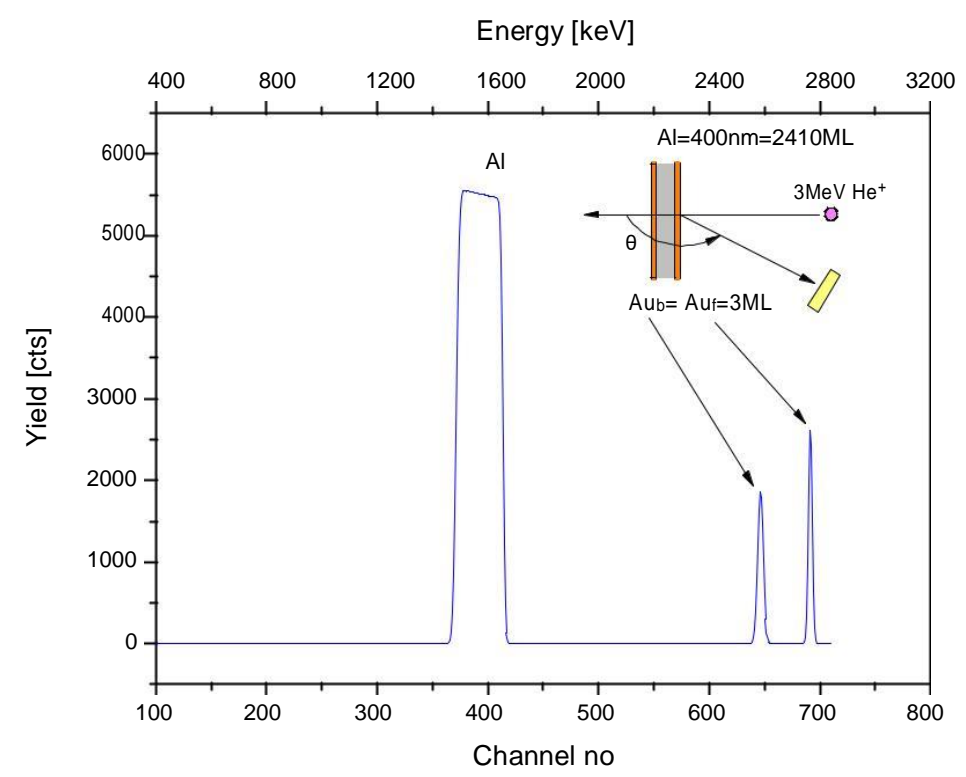

Fig. 7: The backscattering spectrum $\left(\theta=170^{\circ}\right)$ for $3.0 \mathrm{MeV} \mathrm{He}^{+}$ions incident on a 400 $\mathrm{nm}$ Al film with thin Au markers on the front and back surfaces.

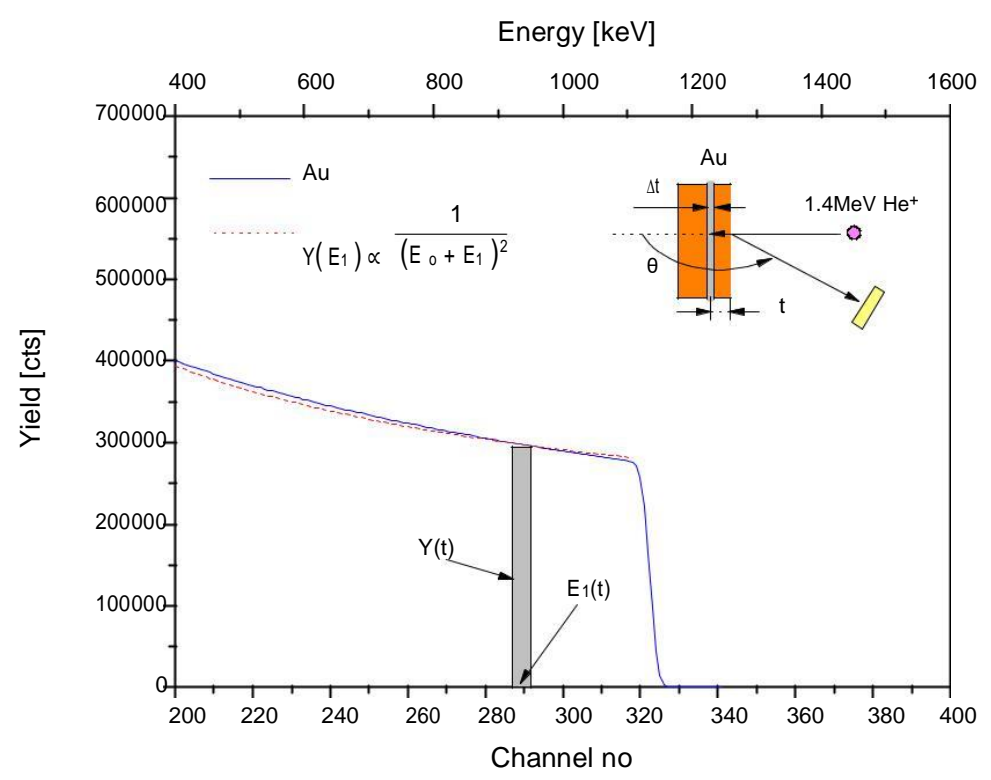

Fig. 8: Backscattered spectrum for $1.4 \mathrm{MeV}^{4} \mathrm{He}^{+}$ions incident on a thick Au sample. The dashed curve is calculated using equation 3.10 and normalised to the experimental curve at 900keV 


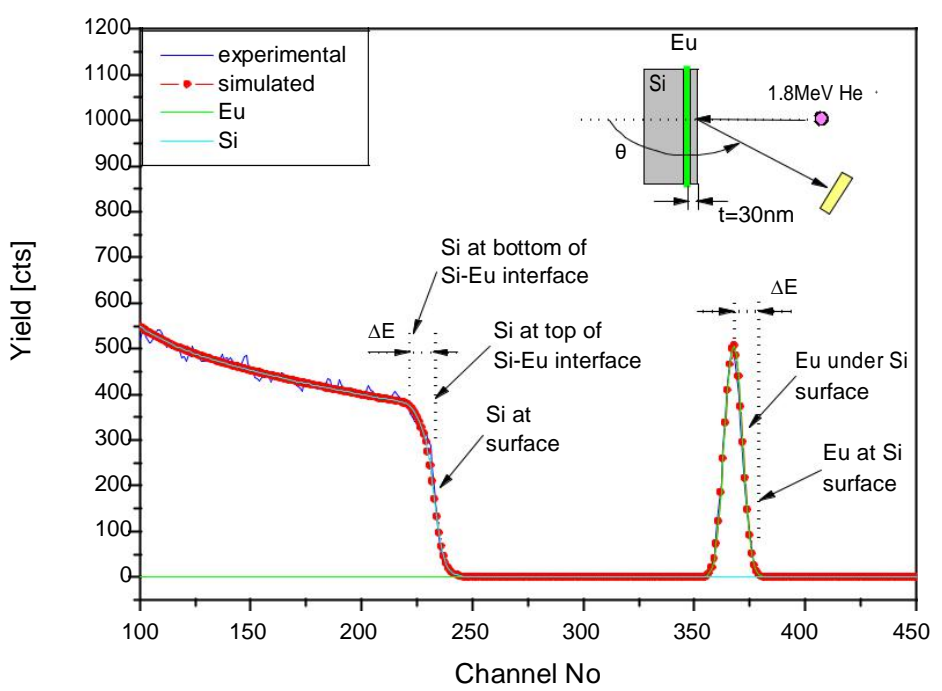

Fig. 9: Energy spectrum of $1.4 \mathrm{MeV}{ }^{4} \mathrm{He}$ ions backscattered from a silicon crystal implanted with a nominal dose of $1.35 \times 10^{16} \mathrm{Eu}$ ions.cm ${ }^{-2}$ at $45 \mathrm{keV}$.

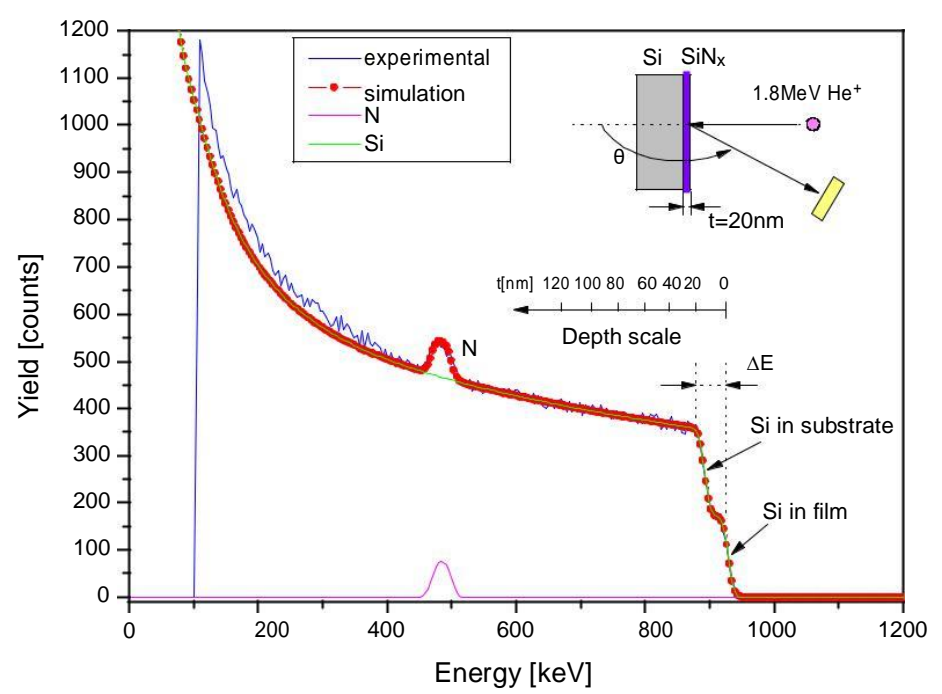

Fig. 10: Schematic backscattering spectra for $1.8 \mathrm{MeV}^{4} \mathrm{He}^{+}$ions incident on a $20 \mathrm{~nm} \mathrm{SiN}_{\mathrm{X}}$ film on a Si substrate (top diagram). Depth scale is indicated below, starting at the surface of the film $(t=0 \mathrm{~nm})$

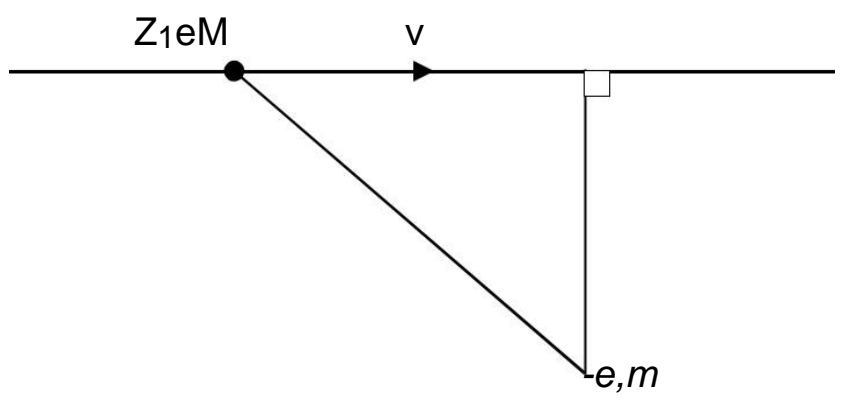

$b$

Fig. 11: Collision between a particle of mass $M$, velocity $v$ with a bound electron of mass $\mathrm{m}$ 


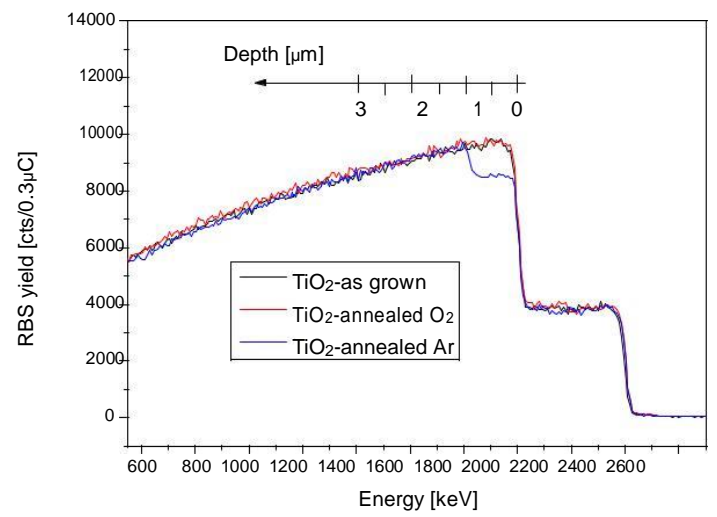

Fig. 12: RBS with $2.5 \mathrm{MeV}$ protons on "as grown" (110) $\mathrm{TiO}_{2}$ single crystal, and on the same crystal annealed in $\mathrm{O}_{2}$ at $900^{\circ} \mathrm{C}$ and $\mathrm{Ar}$ at $600^{\circ} \mathrm{C}$.

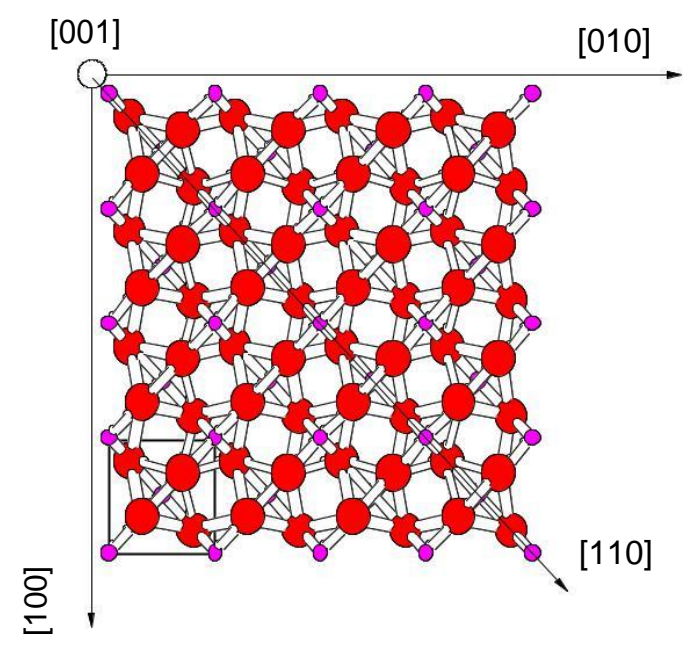

Fig. 13: Schematic representation of basic crystallographic directions in $\mathrm{TiO}_{2}$

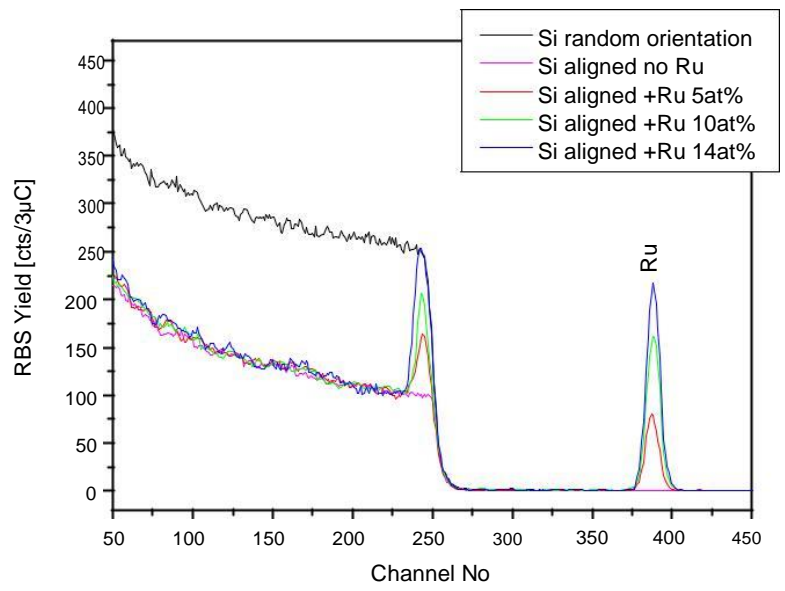

Fig. 14: Channelling RBS on (100) Si implanted with Ru, using 1.8MeV He${ }^{+}$ 


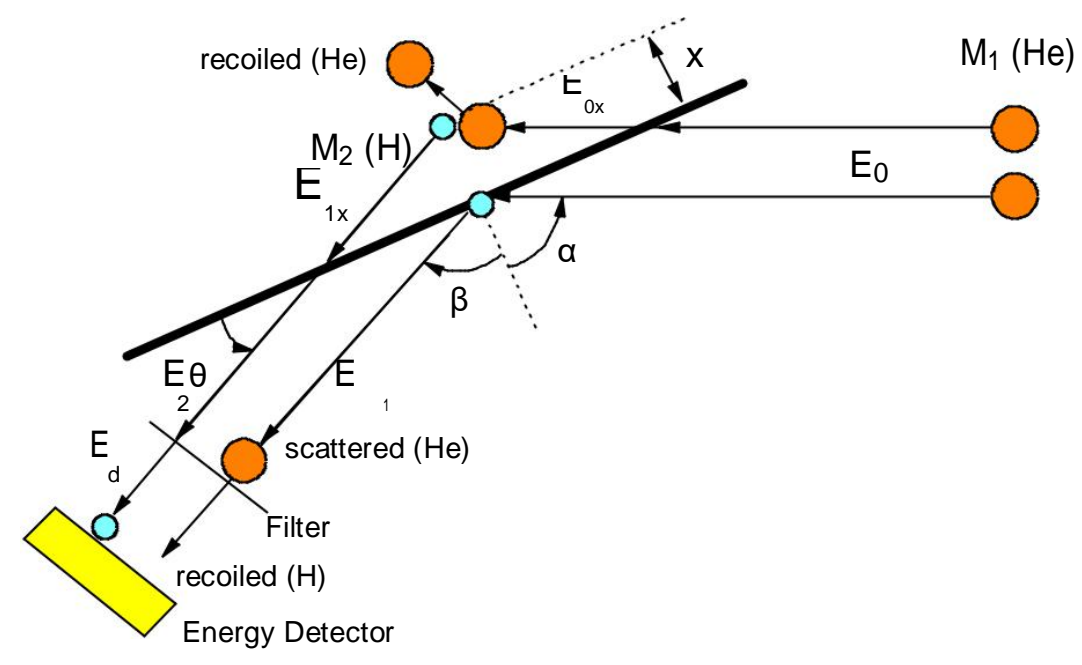

Fig. 15: Schematics of ERDA, using He of primary energy $\mathrm{E}_{0}$. At the surface $\mathrm{H}$ is recoiled with energy $\mathrm{E}_{1} ; \mathrm{E}_{0 \mathrm{x}}$ is the energy loss of $\mathrm{He}$ in the sample between surface and depth $x$, $\mathrm{E}_{1 \mathrm{x}}$ is the energy loss of recoiled $\mathrm{H}$ from depth $x$ to the surface.

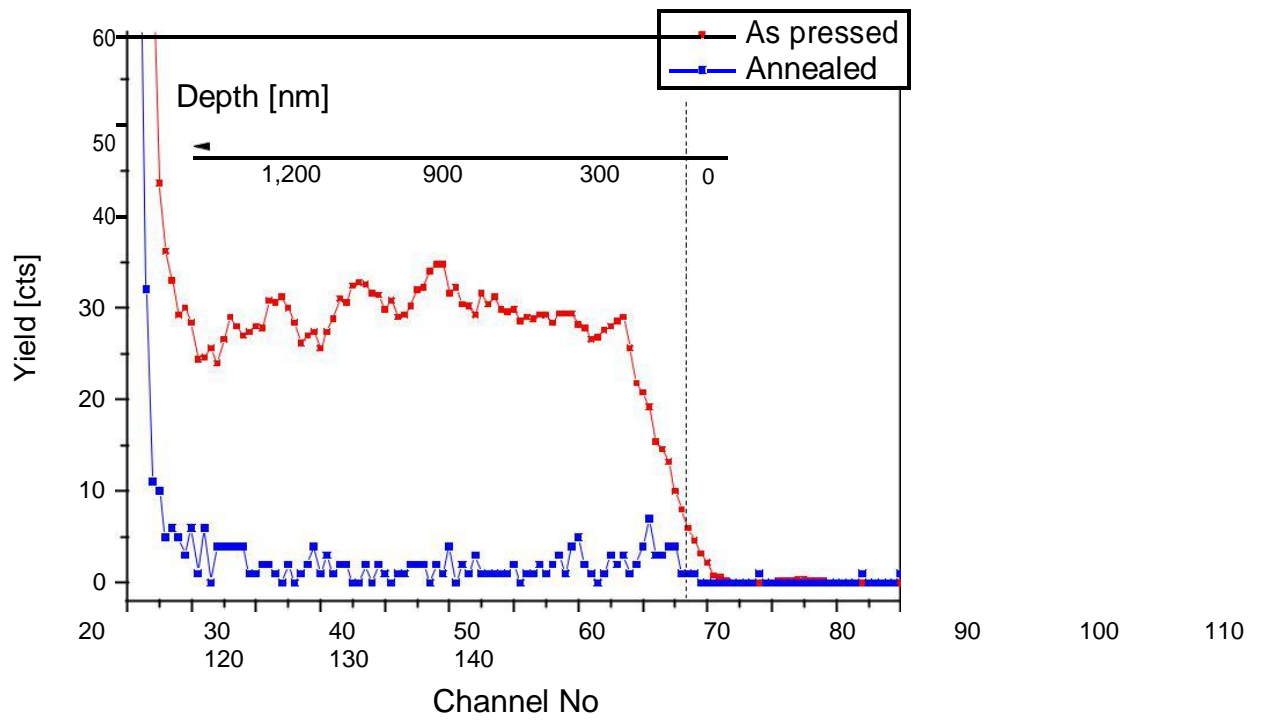

Fig. 16: Hydrogen depth distribution in as pressed polycrystalline $\mathrm{TiO}_{2}$ and pressed and annealed at $1,000^{\circ} \mathrm{C}$ in $\mathrm{Ar}+\mathrm{H}_{2}$ atmosphere, using ERDA method described above, with $2 \mathrm{MeV}{ }^{4} \mathrm{He}$. 


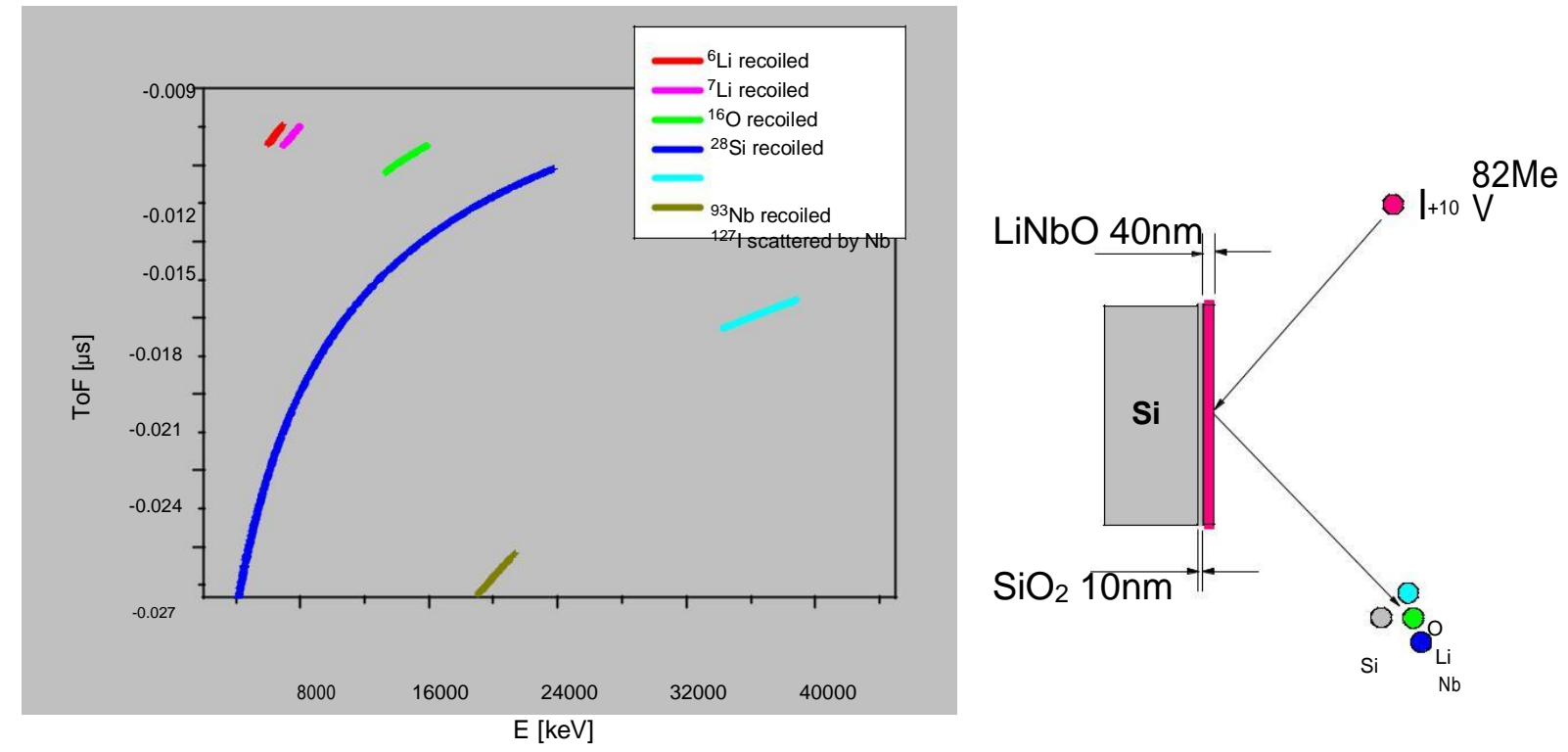

Fig. 17: Typical (t-E) ERDA result obtained with $82 \mathrm{MeV} \mathrm{I}^{+10}$ probe ions for a LiNbO thin film deposited on (100) $\mathrm{Si}$, and a time-of-flight detector; on right is experimental geometry

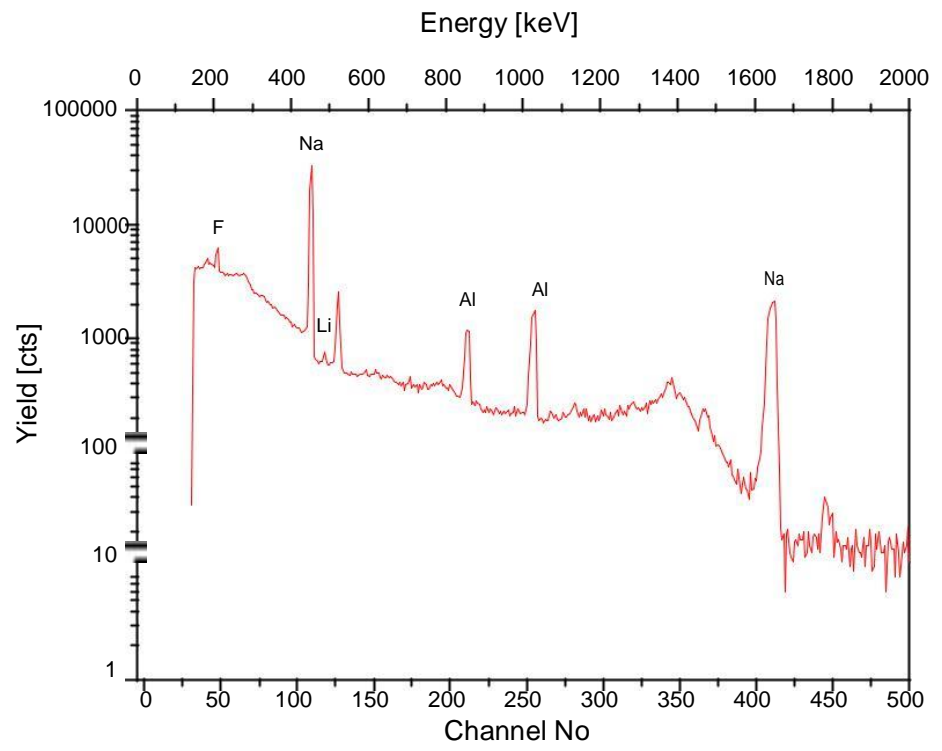

Fig. 18: Typical y-ray spectrum of light elements 


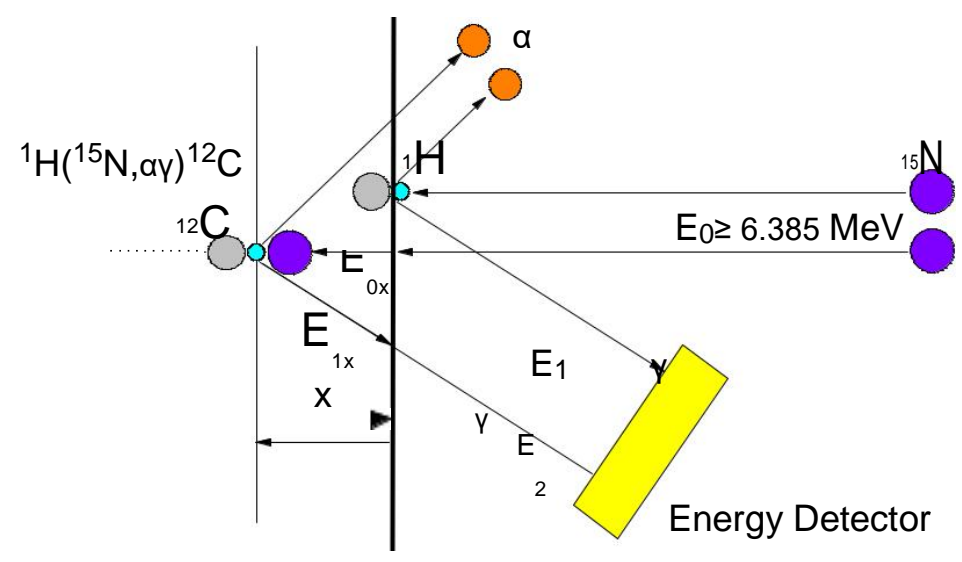

Fig. 19: Schematics of NRA based on ${ }^{15} \mathrm{~N}(\mathrm{H}, \mathrm{a \gamma}){ }^{12} \mathrm{C}$ nuclear reaction, at ${ }^{15} \mathrm{~N}$ resonant energy of $\mathrm{E}_{0}=6.385 \mathrm{MeV}$. $\mathrm{E}_{1}=4.43 \mathrm{MeV}$ is the energy of the resultant prompt $\mathrm{y}$ rays; $\mathrm{E}_{0 \mathrm{x}}$ is the energy loss of ${ }^{15} \mathrm{~N}$ from the surface to the depth $x$; $\mathrm{E}_{1 \mathrm{x}}$ is the energy loss of $\mathrm{y}$ rays from the reaction point to the surface; in most cases $\mathrm{E}_{1 \mathrm{x}}=0$ and $\mathrm{E}_{1}=\mathrm{E}_{2}$

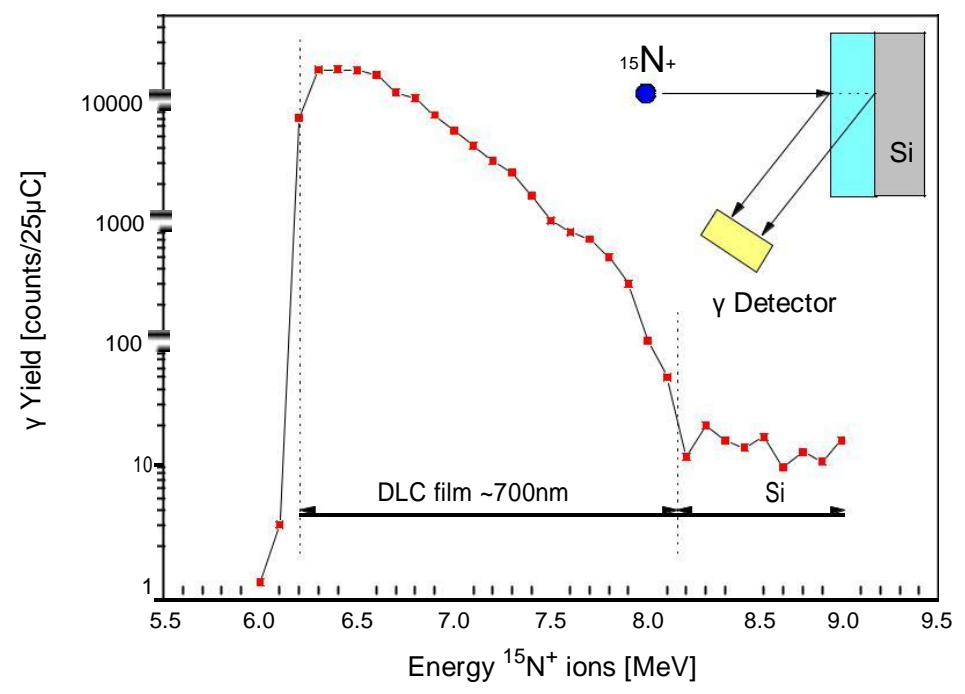

Fig. 20: Hydrogen depth distribution by $15 \mathrm{~N}-\mathrm{NRA}$, in a DLC thick film of around 700nm, deposited on Si. 


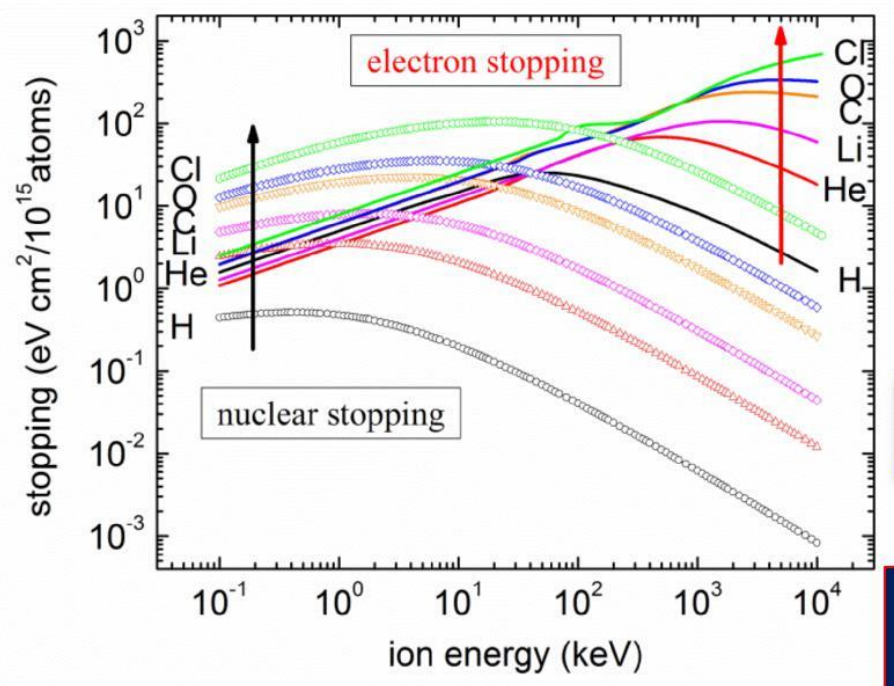

At Higher Energies

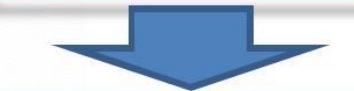

Bremsstrahlung

Cerenkov
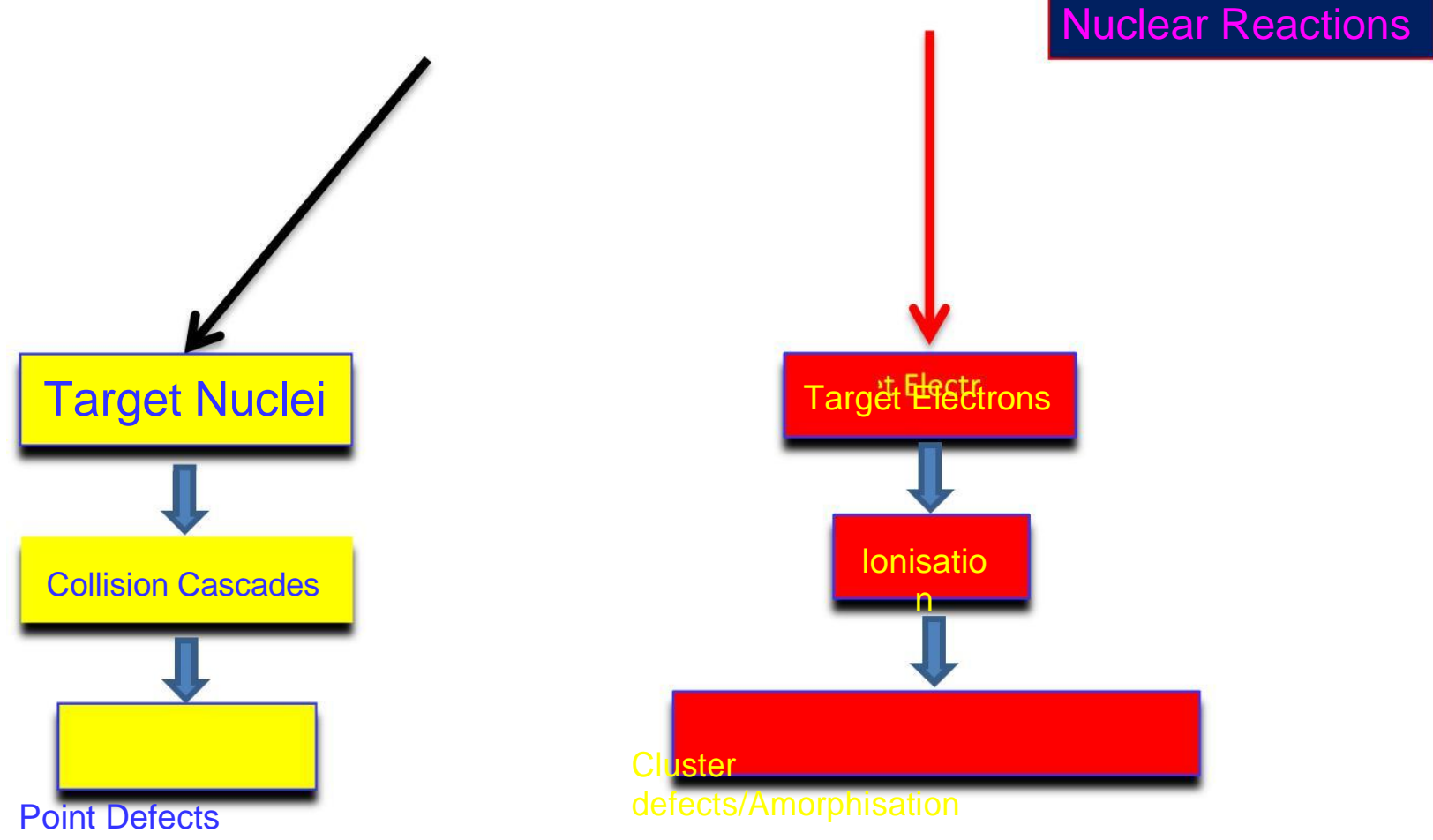

Ionisatio

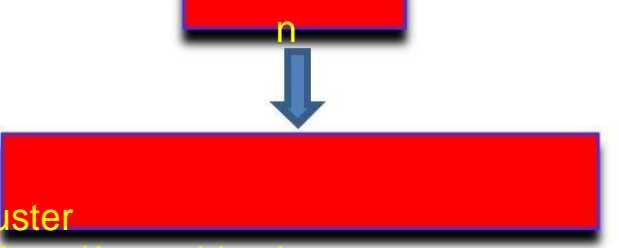

Fig. 21: Calculated stopping power of $\mathrm{Si}$ for light elements $(\mathrm{H}, \mathrm{He}, \mathrm{Li}, \mathrm{C}, \mathrm{O}$ and $\mathrm{Cl})$. Hollow symbols represent nuclear stopping, while lines represent electronic stopping values. 


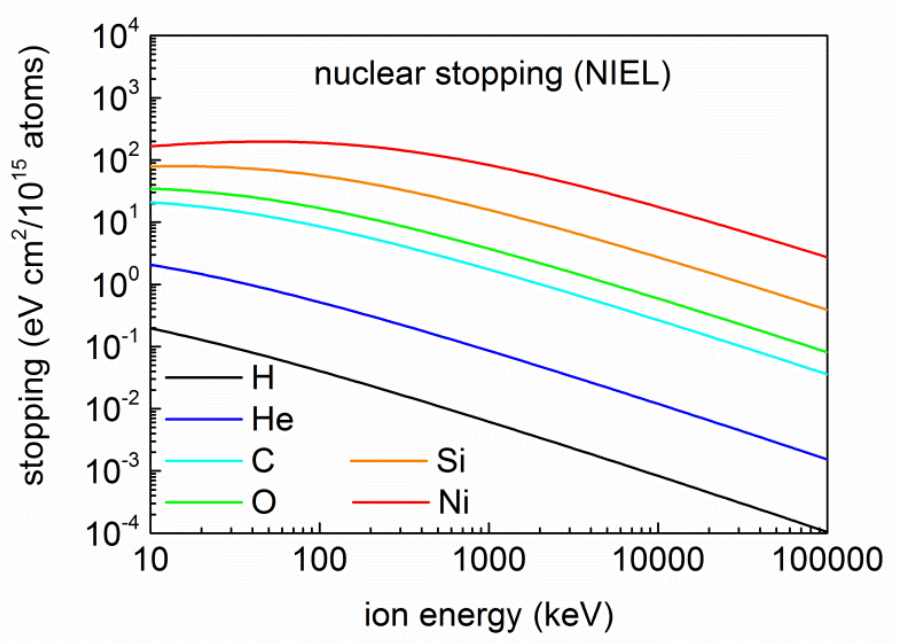

Fig. 22 Nuclear stopping for selected ions in silicon.

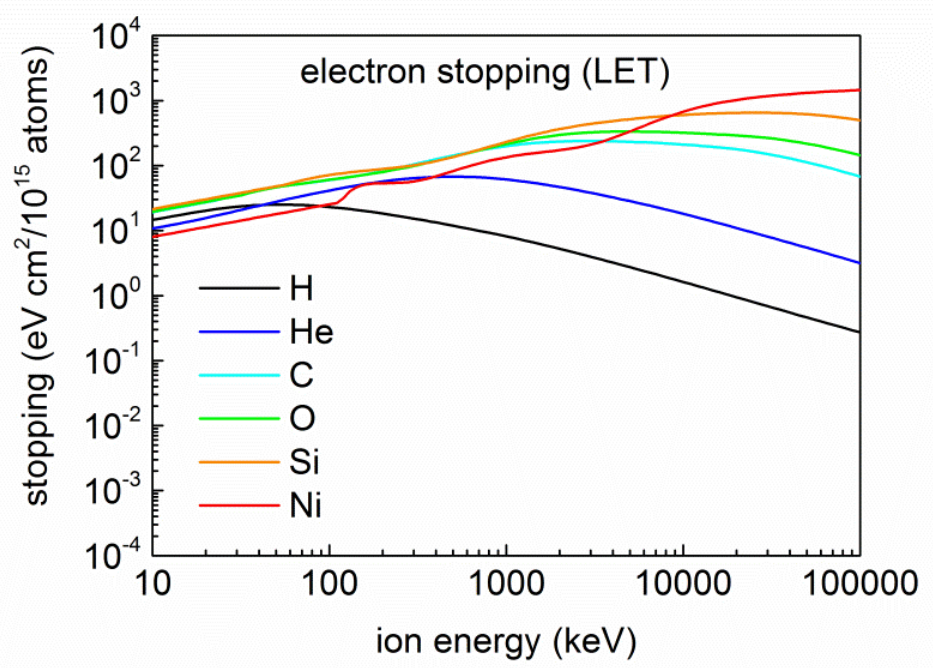

Fig 23: Electronic stopping for selected ions in silicon.

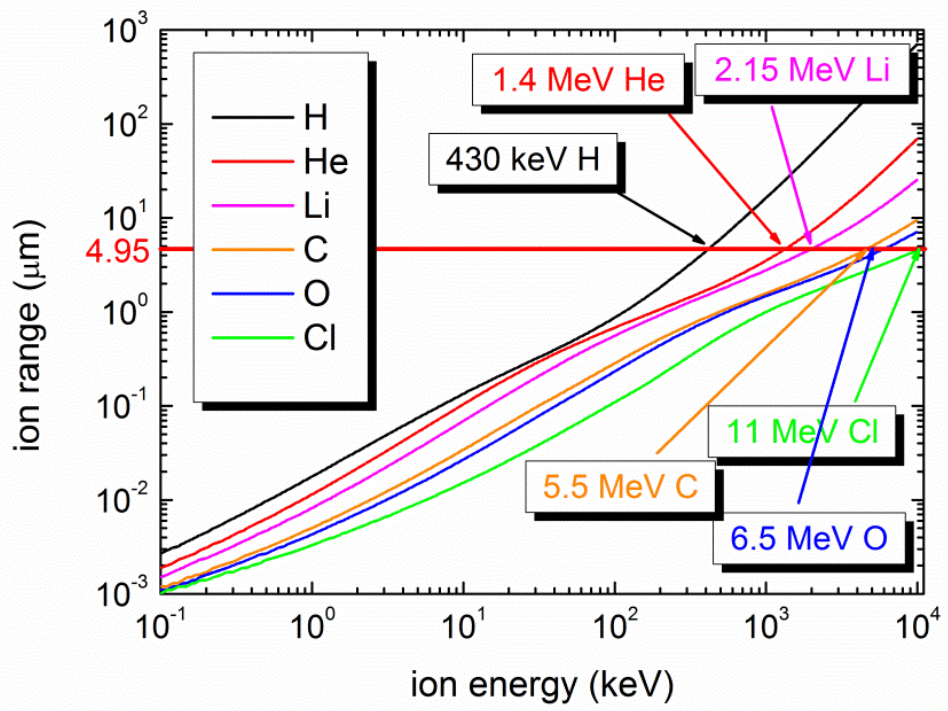

Fig 24: The end of an ion range as a function of the ion incident energy calculated using SRIM. 


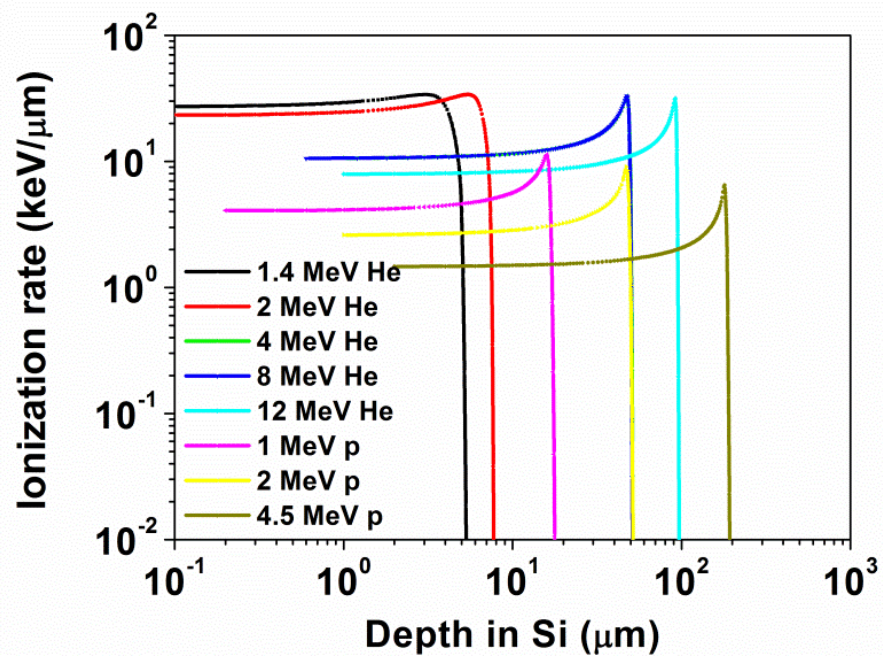

Fig 25: Electronic stopping power, i.e. ionizing energy loss per unit length in silicon for light ions (protons and alpha particles), calculated using SRIM.

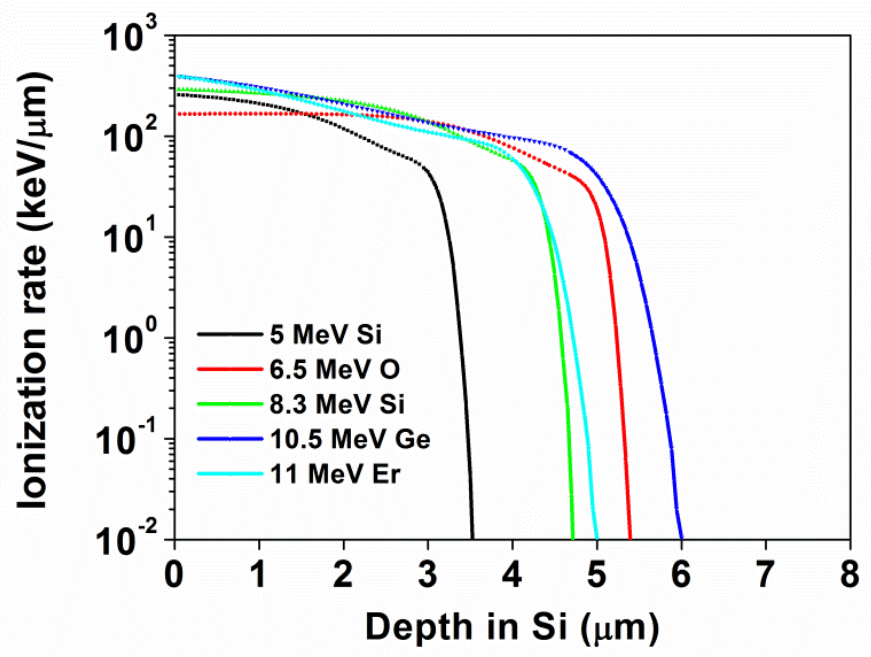

Fig 26: Electronic stopping power in silicon for selected heavy light ions, calculated using SRIM.

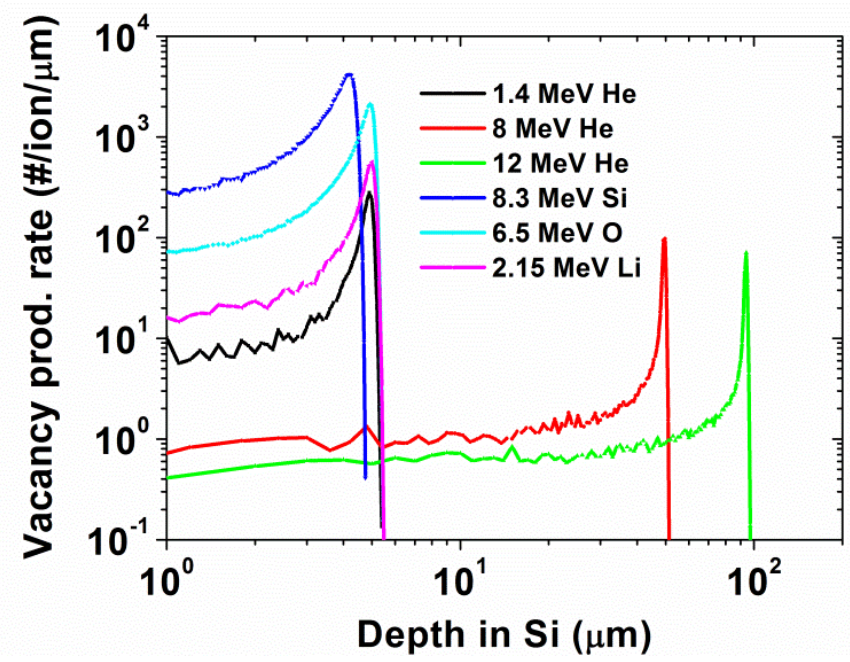

Fig.27: Average vacancy production rate in silicon for selected ions 

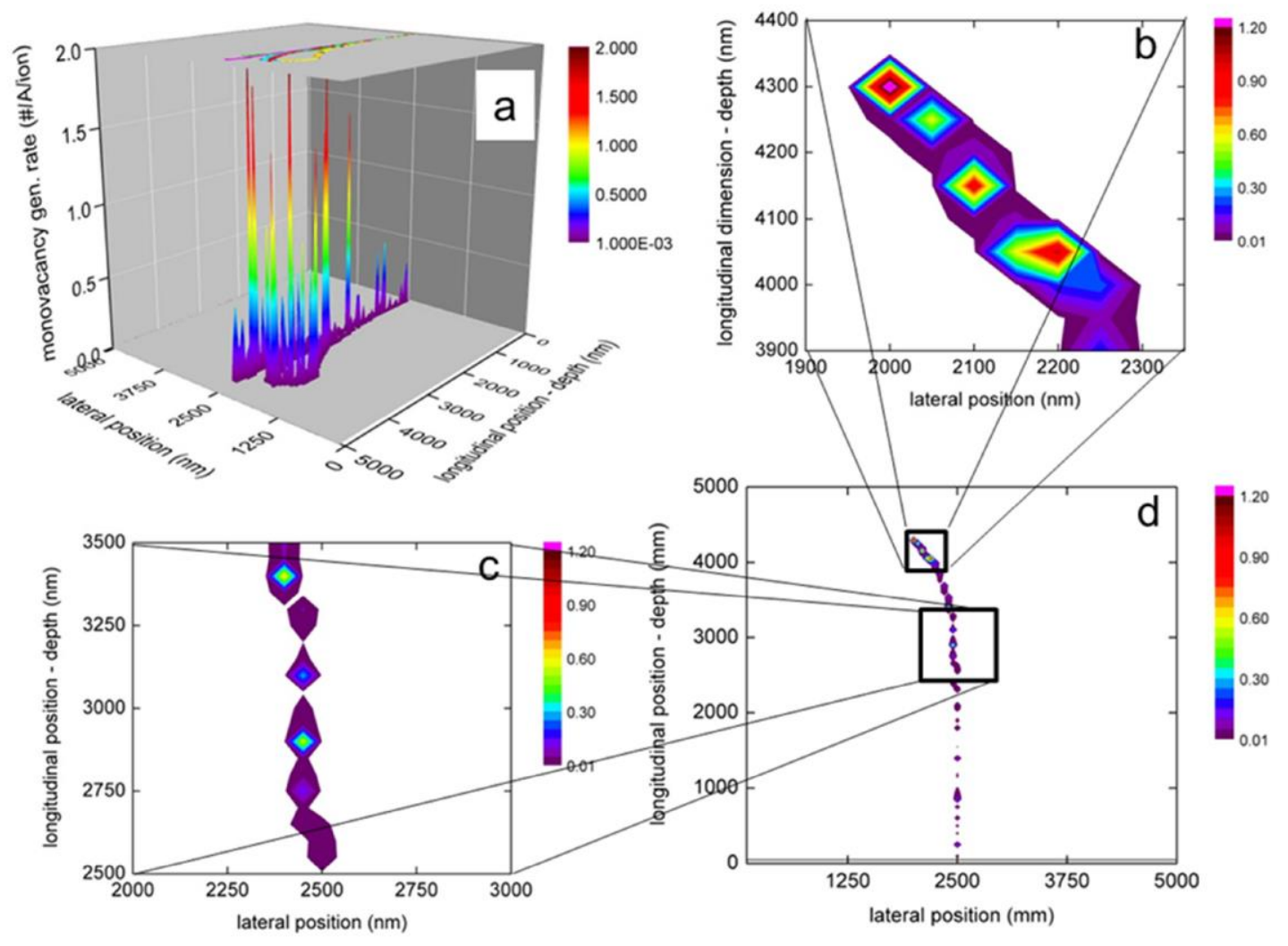

Fig. 28: Monte-Carlo simulation of the disordered region dense with primary defects following the single $8.3 \mathrm{MeV} \mathrm{O}$ ion implantation of silicon: a) the cumulative projection on a horizontal beam plane of all monovacancies generated per unit length of a projectile (VGR) for five implanted ions with entry point at $(2500,0) \mathrm{nm}$; d) the surface contour plot of the VGR distribution generated by random chosen single ion cascade in $\mathrm{Si}$; b) the enlarged disordered area close to the end of an ion range, and c) the enlarged midrange area corresponding to the implant tail. VGR yields shown by colour bars are expressed in monovacancy per Angstrom units.

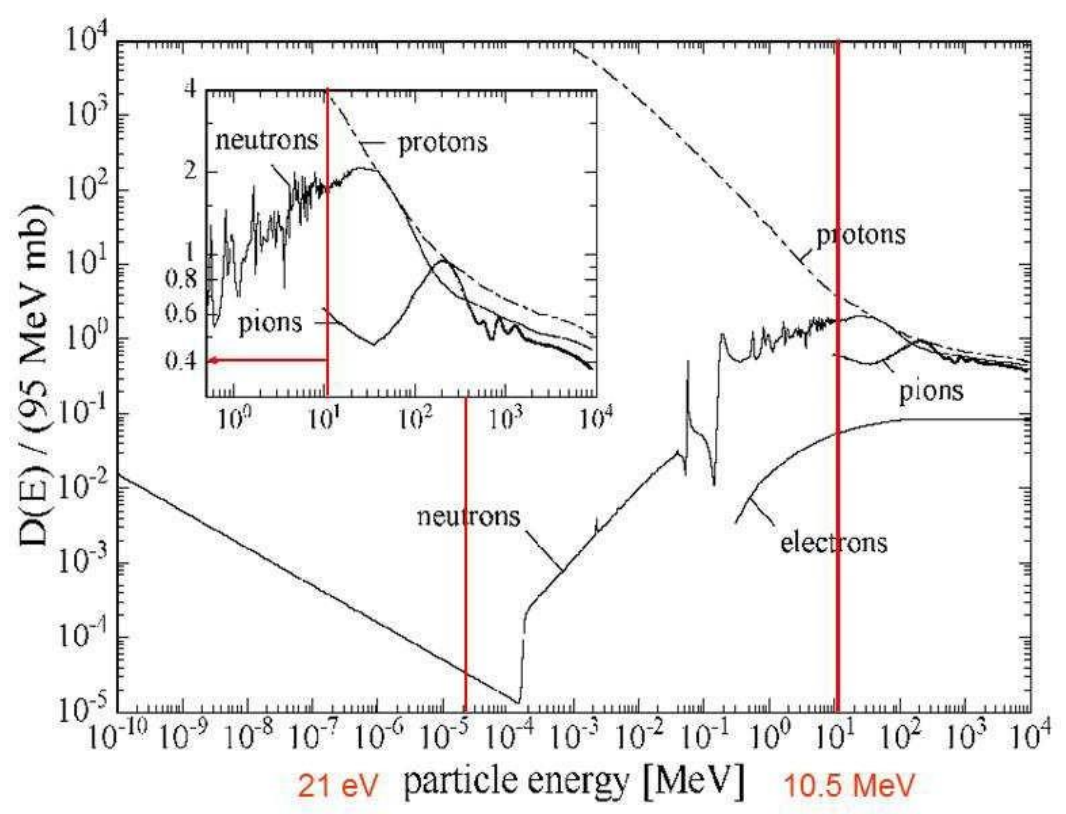

Fig. 29: Non ionizing energy loss of particles in the energy range common to environments with harsh ionizing radiation conditions [25]. 

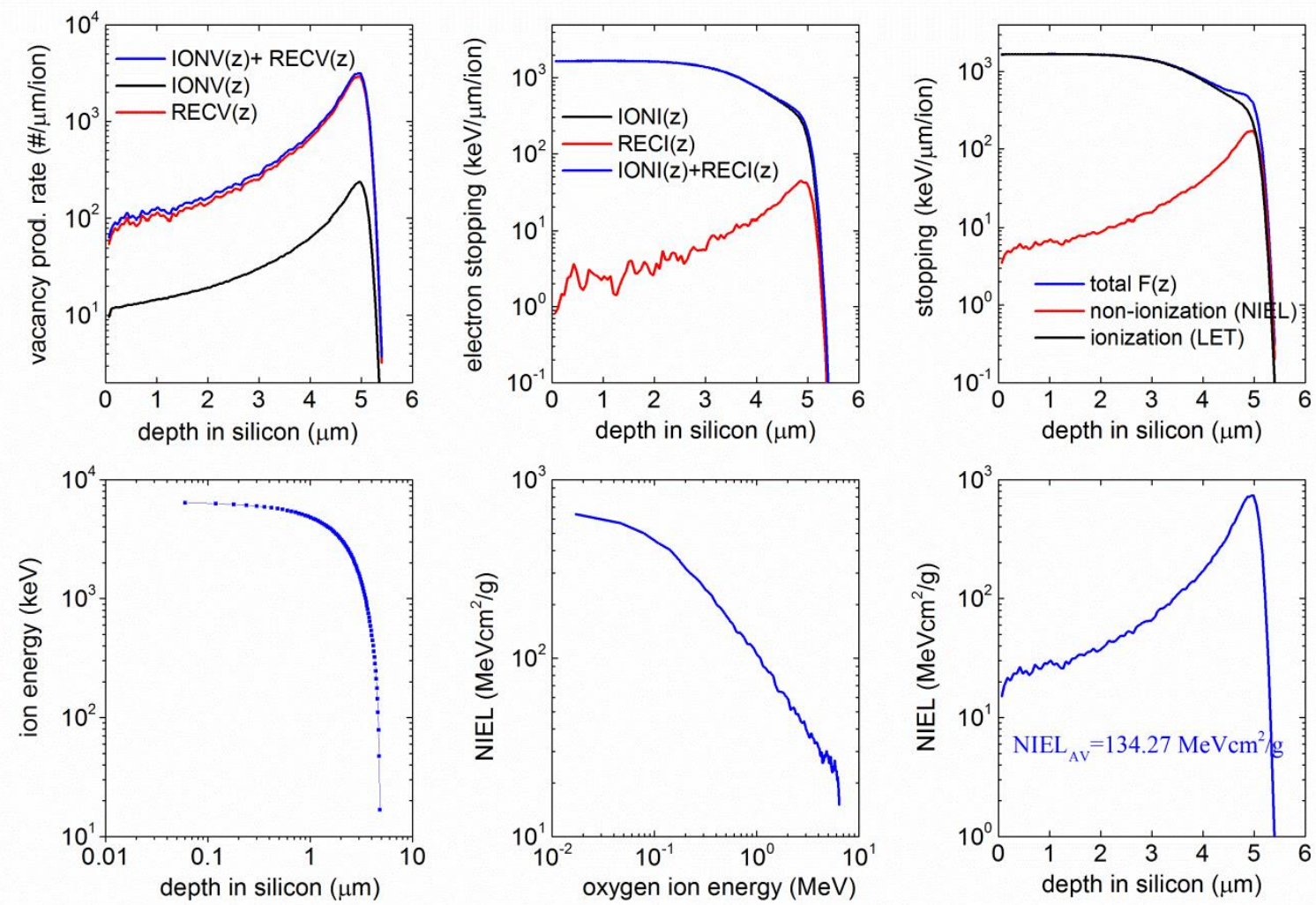

Fig 30: Calculated non-ionizing energy loss depth profile NIEL(z) for $6.5 \mathrm{MeV} O$ ion in silicon. Is shown together with the average NIEL value (NIELAV).

Table 2: The basic semiconductor properties of some semiconductor materials

\begin{tabular}{|c|c|c|c|c|c|}
\hline Property & $\mathrm{TiO}_{2}$ & $\mathrm{Si}$ & $\mathrm{SiC}$ & GaAs & $\begin{array}{l}\text { Diamon } \\
\mathrm{d}\end{array}$ \\
\hline Structure & tetragonal & cubic & Hexagonal & $\begin{array}{l}\text { Zinc } \\
\text { blende }\end{array}$ & $\begin{array}{l}\text { Diamon } \\
d\end{array}$ \\
\hline $\begin{array}{l}\text { Mass Density } \\
{\left[\mathrm{g} / \mathrm{cm}^{3}\right]}\end{array}$ & $\begin{array}{c}4.2 \\
3\end{array}$ & 2.33 & $\begin{array}{c}3.2 \\
1\end{array}$ & 5.32 & 3.52 \\
\hline $\begin{array}{l}\text { Atomic Density } \\
{\left[10^{22} \mathrm{at} / \mathrm{cm}^{3}\right]}\end{array}$ & $\begin{array}{c}6.4 \\
2\end{array}$ & 5 & 9.7 & 4.43 & 18 \\
\hline Band Structure & Direct & Indirect & Indirect & Direct & Indirect \\
\hline Band Gap [eV] & $\begin{array}{c}3.0 \\
2 \\
\end{array}$ & 1.12 & $\begin{array}{c}3.2 \\
6 \\
\end{array}$ & 1.43 & 5.45 \\
\hline $\begin{array}{c}\text { Dielectric Constant } \\
\left(\mathrm{e}_{\mathrm{r}}\right)\end{array}$ & 10 & 11.7 & $\begin{array}{c}9.6 \\
6\end{array}$ & 10.9 & 5.68 \\
\hline $\begin{array}{c}\text { Energy to make e- } \\
\mathrm{h} \\
{[\mathrm{eV}]}\end{array}$ & & 3.62 & $\begin{array}{c}7.7 \\
8\end{array}$ & 4.21 & 13.2 \\
\hline $\begin{array}{r}\text { Displacement } \\
\text { energy [eV/at] }\end{array}$ & 18 & 21 & 35 & 10 & 50 \\
\hline $\begin{array}{l}\text { Electron mobility } \\
{\left[\mathrm{cm}^{2} / \mathrm{V} \mathrm{s}\right]}\end{array}$ & 1 & $\begin{array}{l}1,400- \\
1,500\end{array}$ & $800-1,000$ & 8,500 & $\begin{array}{l}1,800- \\
2,200\end{array}$ \\
\hline $\begin{array}{l}\text { Hole mobility } \\
{\left[\mathrm{cm}^{2} / \mathrm{V} \mathrm{s}\right]}\end{array}$ & & $450-600$ & $100-120$ & 400 & $\begin{array}{l}1,200- \\
1,600 \\
\end{array}$ \\
\hline $\begin{array}{l}\text { Diff. Coefficient e } \\
{\left[\mathrm{cm}^{2} / \mathrm{s}\right]}\end{array}$ & & $<36$ & $<22$ & $<200$ & $<57$ \\
\hline
\end{tabular}




\begin{tabular}{|c|c|c|c|c|c|}
\hline $\begin{array}{c}\text { Therm. Velocity e } \\
{\left[10^{7} \mathrm{~cm} / \mathrm{s}\right]}\end{array}$ & $4.8-11.8$ & 2.3 & 1.9 & 4.4 & $\sim 1$ \\
\hline $\begin{array}{c}\text { e-sat. Velocity } \\
{\left[10^{7} \mathrm{~cm} / \mathrm{s}\right]}\end{array}$ & $\sim 1,500$ & $0.8-1$ & $0.8-2.2$ & $1-2$ & $2.2-2.7$ \\
\hline $\begin{array}{c}\text { Breackdown Field } \\
{\left[10^{5} \mathrm{~V} / \mathrm{cm}\right]}\end{array}$ & 1,843 & $2-3$ & $22-40$ & $3-6$ & 100 \\
\hline $\begin{array}{c}\text { Therm. } \\
\text { Conductivity } \\
\text { Ith }[\mathrm{W} / \mathrm{cm} \mathrm{K}]\end{array}$ & 20 & 1.5 & $3-5$ & 0.55 & 20 \\
\hline $\begin{array}{c}\text { Max. Operating } \\
\text { temp }\left[{ }^{\circ} \mathrm{C}\right]\end{array}$ & 300 & 1,200 & & 1,100 \\
\hline Melting point [ $\left.{ }^{\circ} \mathrm{C}\right]$ & excellent & 1,420 & 1,800 & & 3,500 \\
\hline Air Stability & Good & Excellent & fair & $\begin{array}{c}\text { Excellen } \\
\mathrm{t}\end{array}$ \\
\hline Water stability & excellent & poor & fair & poor & $\begin{array}{c}\text { Excellen } \\
\mathrm{t}\end{array}$ \\
\hline
\end{tabular}

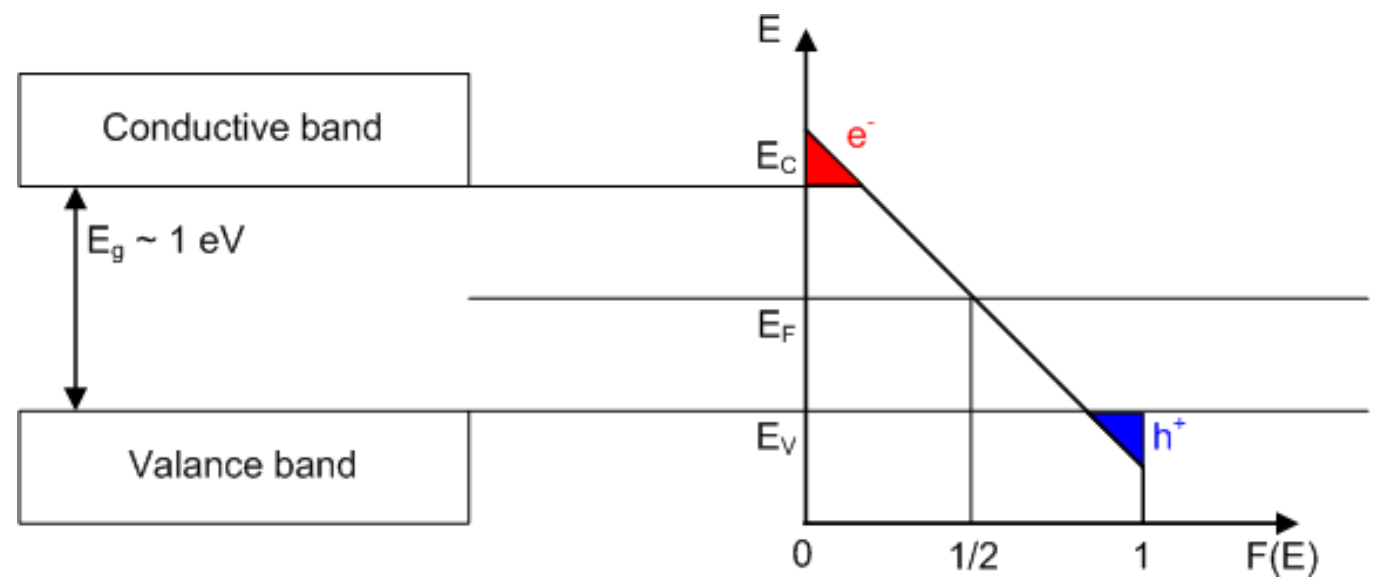

Fig 31: Density of energy states distribution function for intrinsic semiconductor at a temperature $T(T>0)$. The hole occupied states, in the valance band are shown in blue $(F(E)<1)$, while the electron occupied energy states in the conductive band are shown in red $(F(E)>0)$.

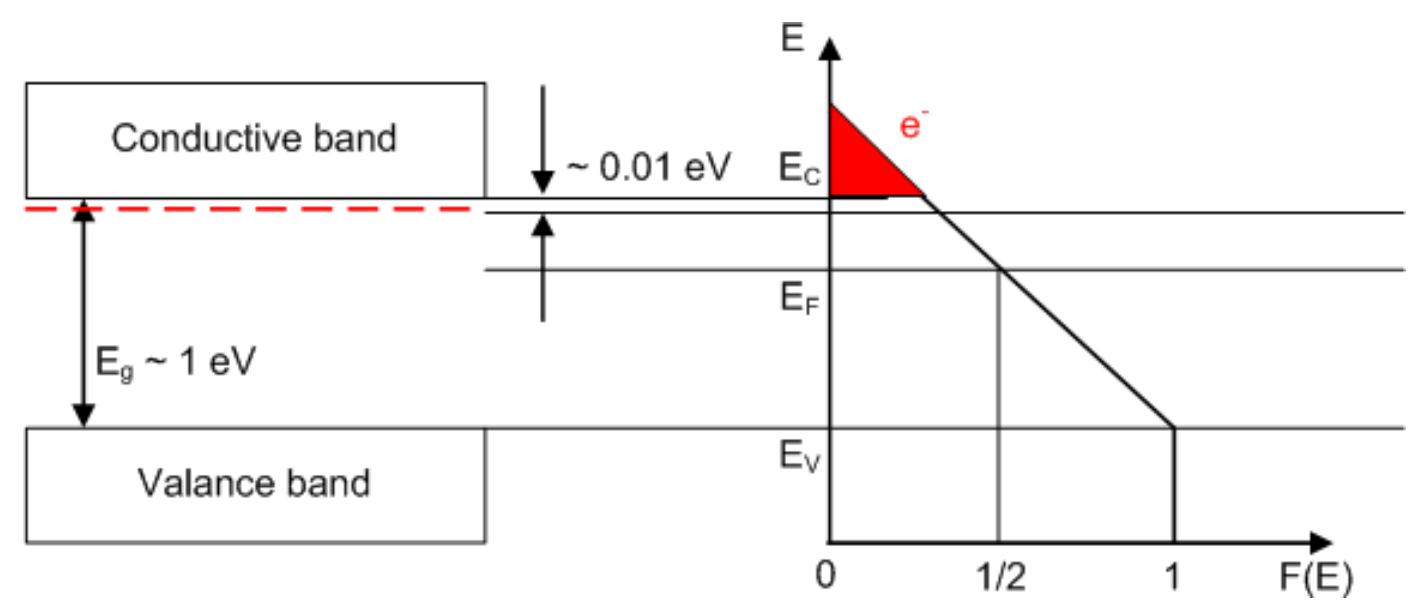

Fig 32: Density of energy states distribution function for the $n$-type semiconductor $(T>0)$. 


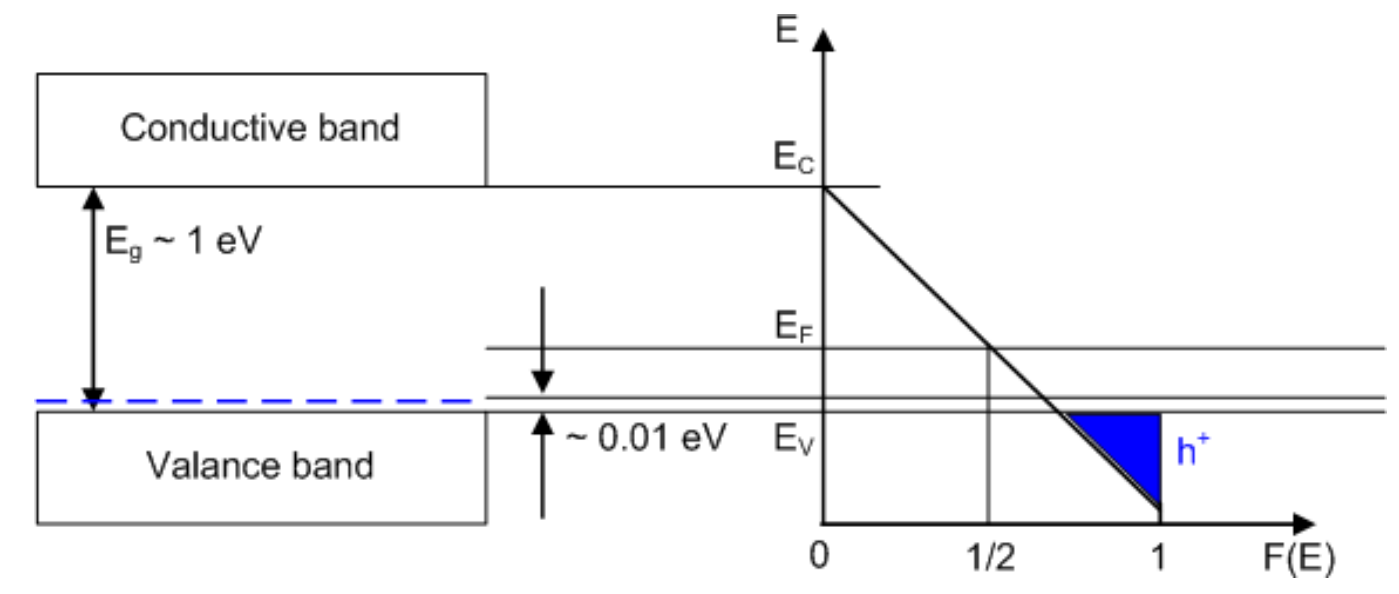

Fig 33: Density of energy states distribution function for the $p$-type semiconductor $(T>0)$.
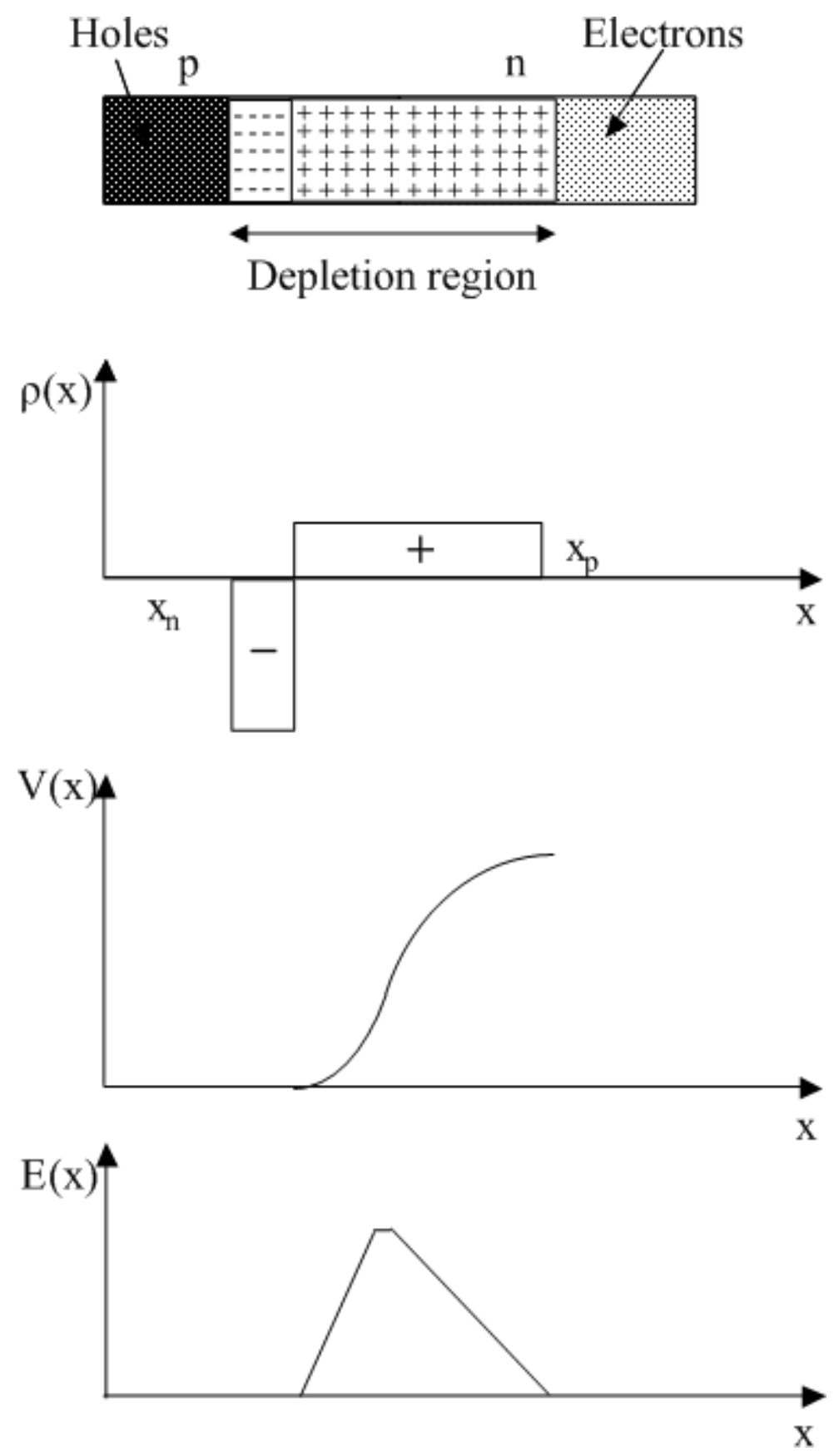

Fig 34: One dimensional schematics of the space charge, electric potential and 
electric field strength distribution across a p-n junction, where (a) is at the top and (d) at the bottom.

Fig 35: Primary damage in semiconductor crystals-the vacancy and interstitial pair.

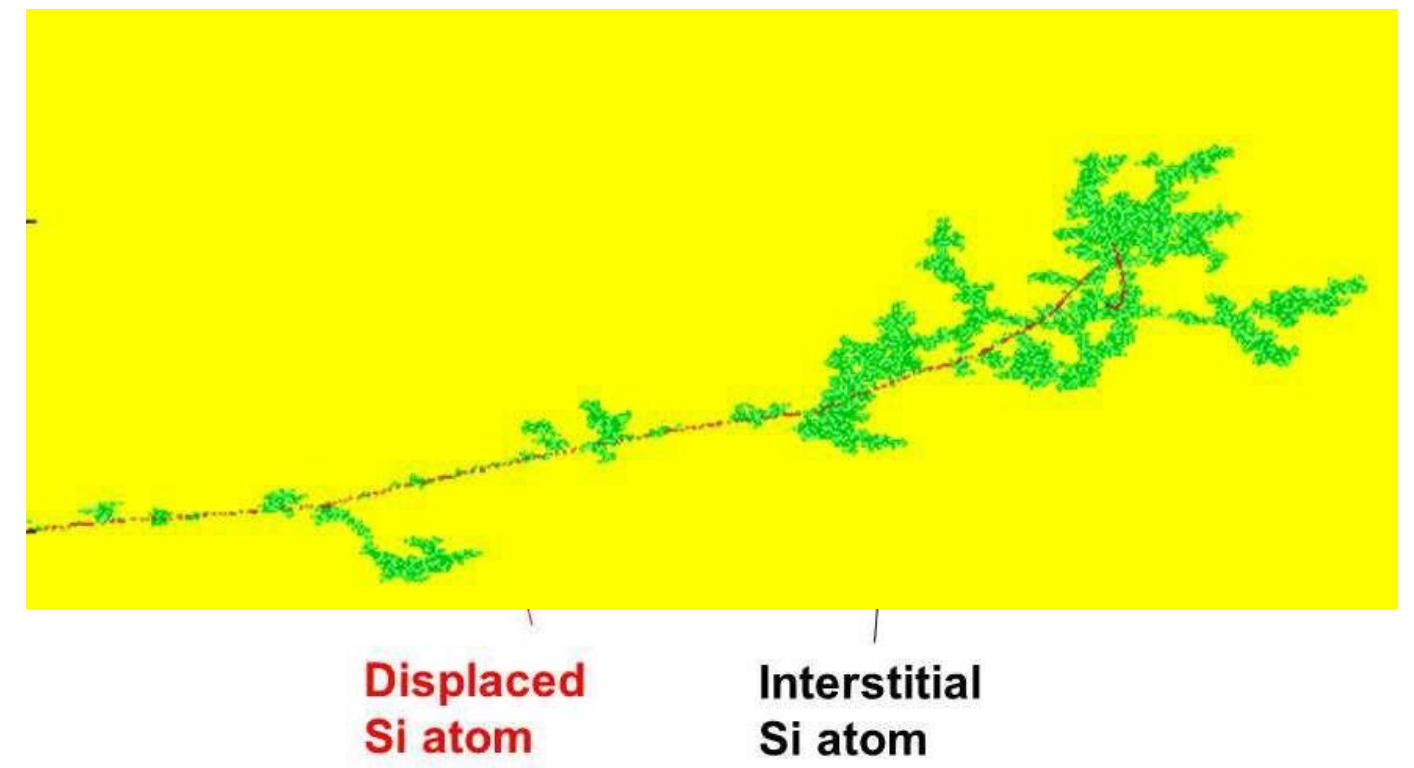

Fig 36: Simulation of a cascade showing ununiform distribution of vacancies and interstitials created by the $35 \mathrm{keV}$ silicon ion projectile impiging in silicon.

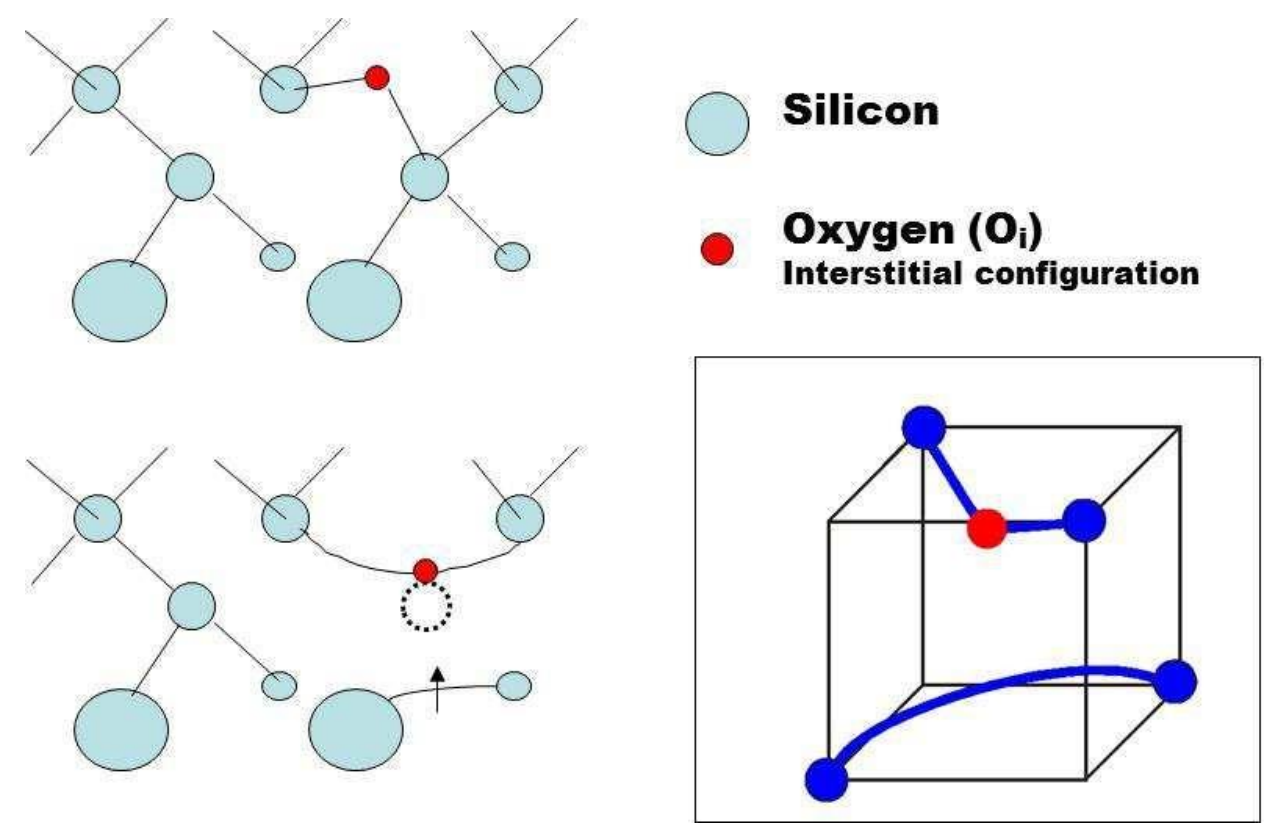

Fig 37: VO complex configuration 


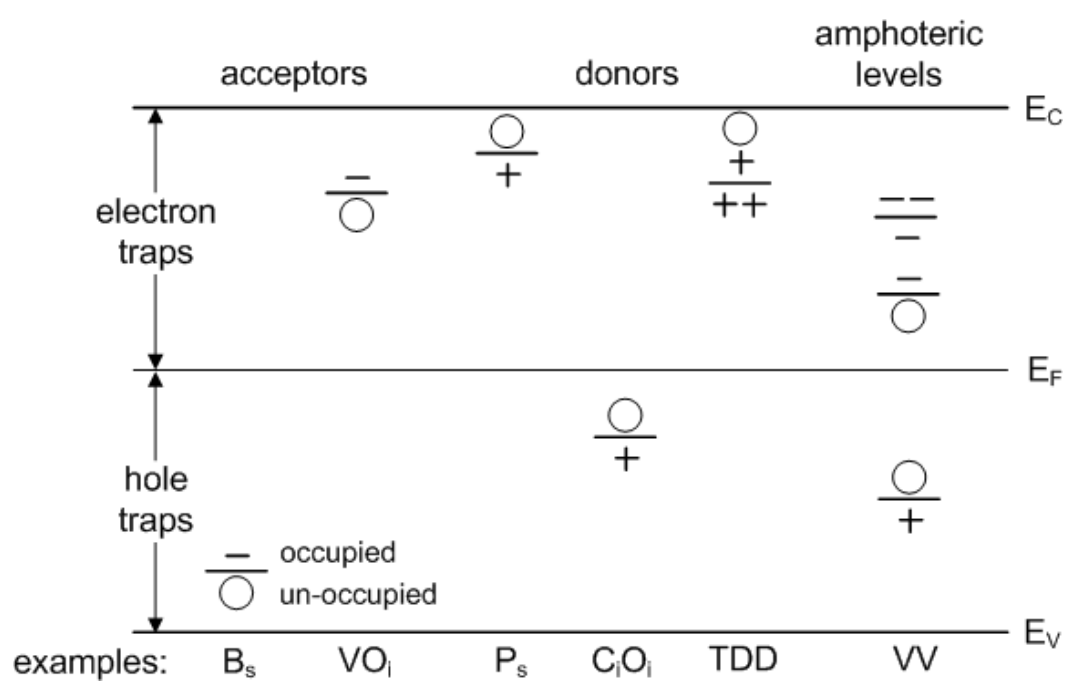

Fig 38: Different charge states (+ positive unit charge, 0 neutral charge state, - negative unit charge) for acceptor, donor and amphoteric states in the silicon band-gap

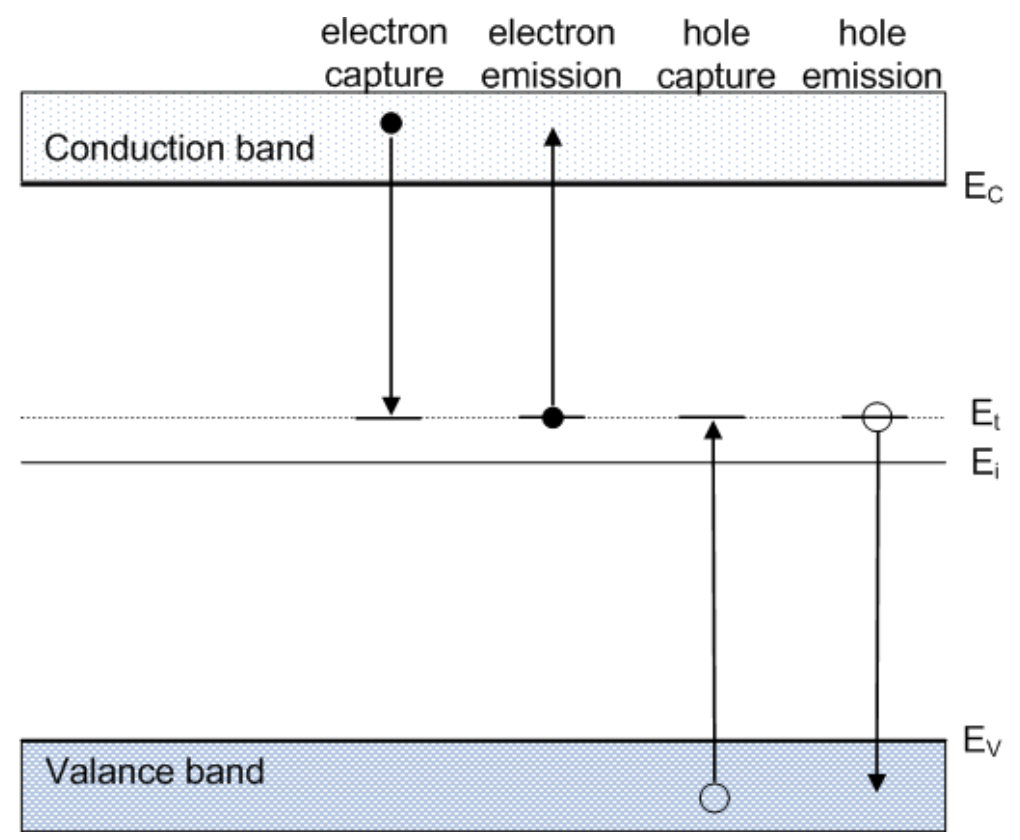

Fig 39: Charge exchange processes between the states in valence/conductive band and the states within energy band-gap in semiconductor described by the ShockleyRead-Hall statistics. 


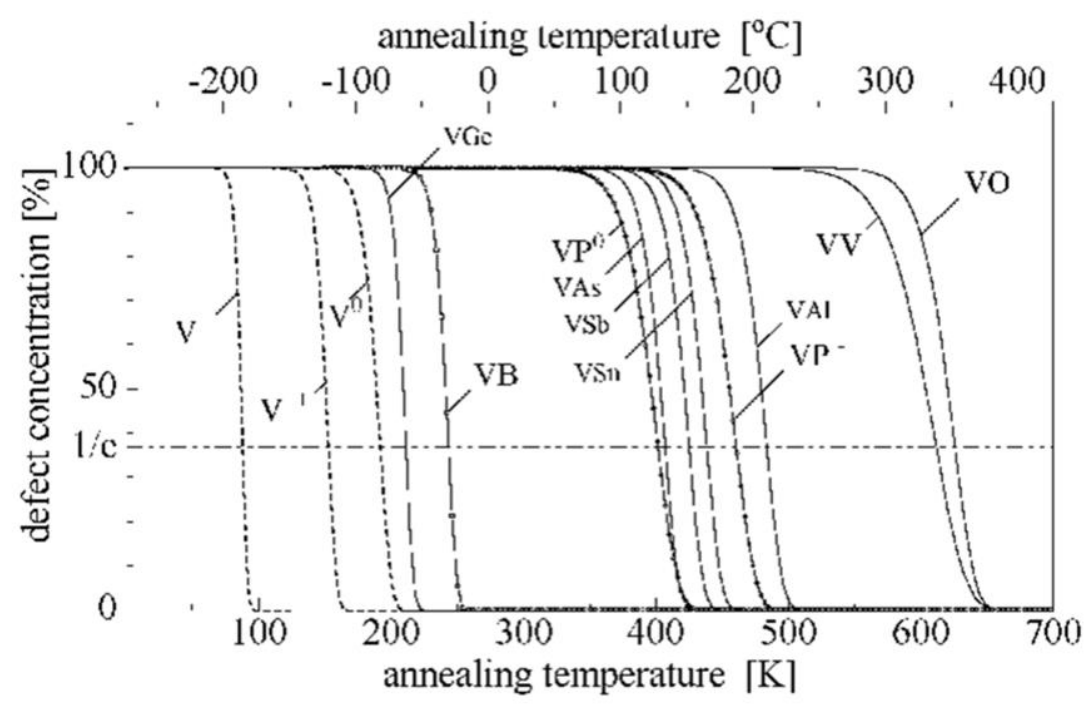

Fig 40: Relat ive abundance of vacancy related defects as a function of temperature.

Table 3: Vacancy related point defects in $n$-type silicon and their thermal activation energies, annealing temperatures and energies levels in a repect to the band edges, relations (51) and (52).

\begin{tabular}{|c|c|c|c|c|}
\hline Defect & Eann (Tann) & Ref & $\Delta \mathrm{H}$ & Ref \\
\hline$V^{--}$ & $0.18 \mathrm{eV}$ & [46] & & \\
\hline$v^{-}$ & $0.18 \mathrm{eV}$ & [46] & & \\
\hline$v^{0}$ & $0.45 \mathrm{eV}$ & {$[46]$} & $+0.05 \mathrm{eV}$ & \\
\hline $\mathrm{V}^{+}$ & negativan-U & {$[46]$} & & \\
\hline $\mathrm{V}^{++}$ & $0.32 \mathrm{eV}$ & [46] & $+0.13 \mathrm{Ev}$ & \\
\hline$v_{2}{ }^{--}$ & & & & \\
\hline $\mathrm{V}_{2}{ }^{-}$ & & & $-0.235 \mathrm{eV}$ & [48] \\
\hline $\mathrm{V}_{2}{ }^{\mathrm{O}}$ & $1.47 \mathrm{eV}(340)$ & [47] & $-0.420 \mathrm{eV}$ & [48] \\
\hline$v_{2}{ }^{+}$ & & & $+0.196 \mathrm{eV}$ & [48] \\
\hline $\mathrm{VO}^{-}$ & & & & \\
\hline $\mathrm{VO}_{\mathrm{i}}$ & $2.27 \mathrm{eV}(350)$ & [49] & -0.164 & [48] \\
\hline $\mathrm{VP}^{-}$ & $1.25 \mathrm{eV}(190)$ & [50] & & \\
\hline VP0 & $0.95 \mathrm{eV}(130)$ & [50] & $-0.456 \mathrm{eV}$ & [51] \\
\hline
\end{tabular}




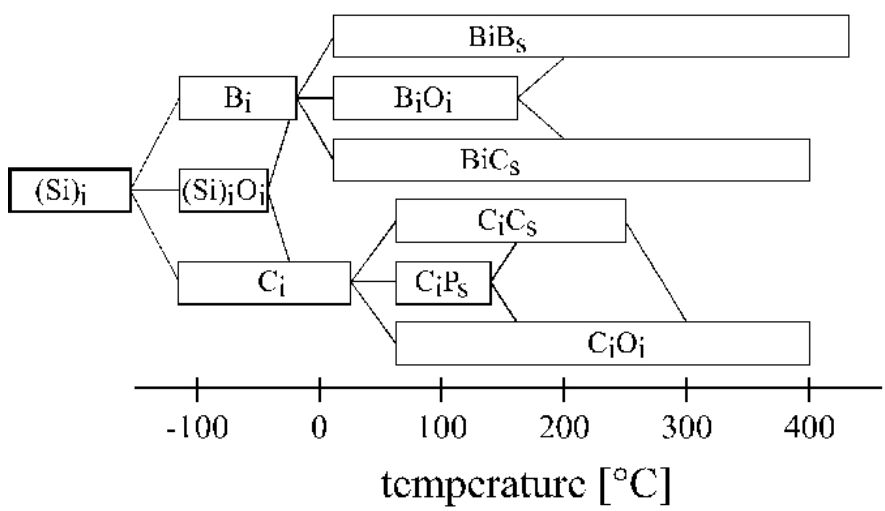

Fig 41: Temperature dependence of interstitial defects in $p$-type silicon

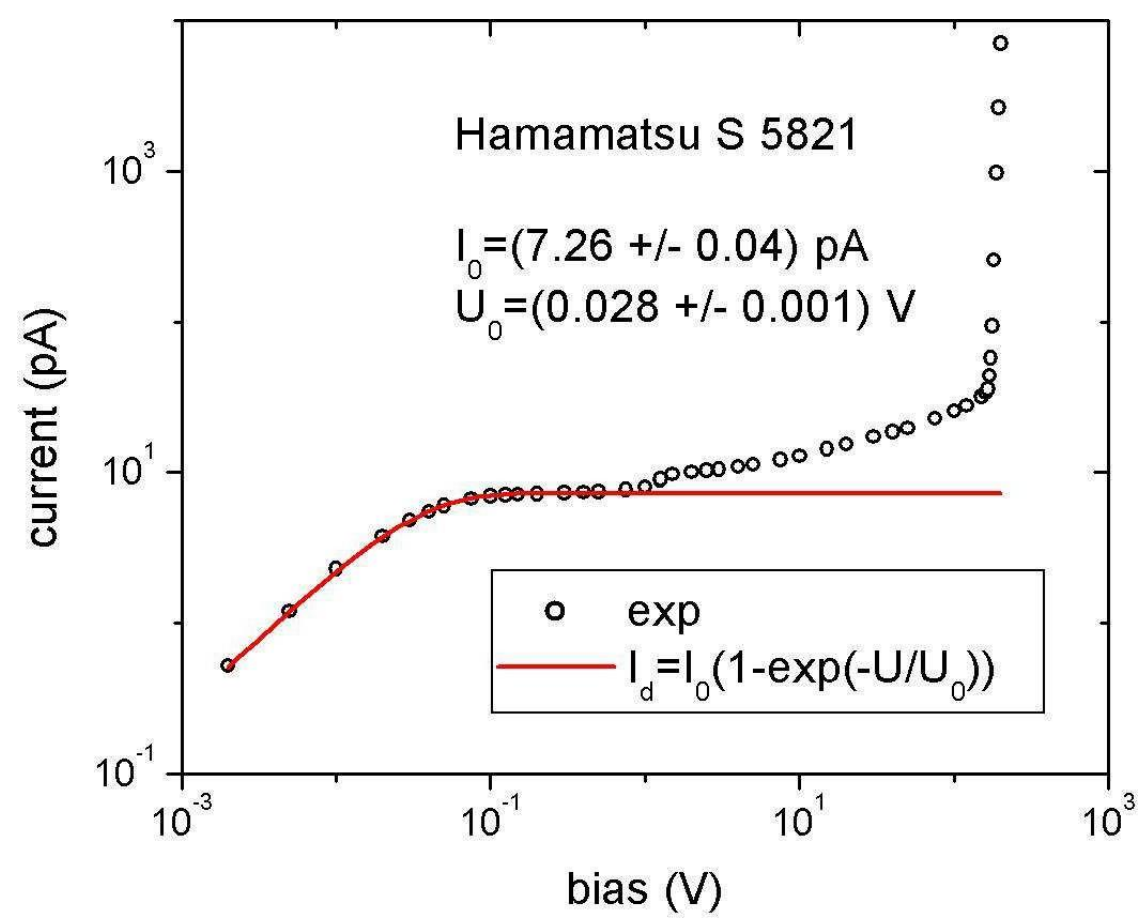

Fig 42: I-V characteristic for a pristine silicon PIN photodiode (Hamamatsu S5821).

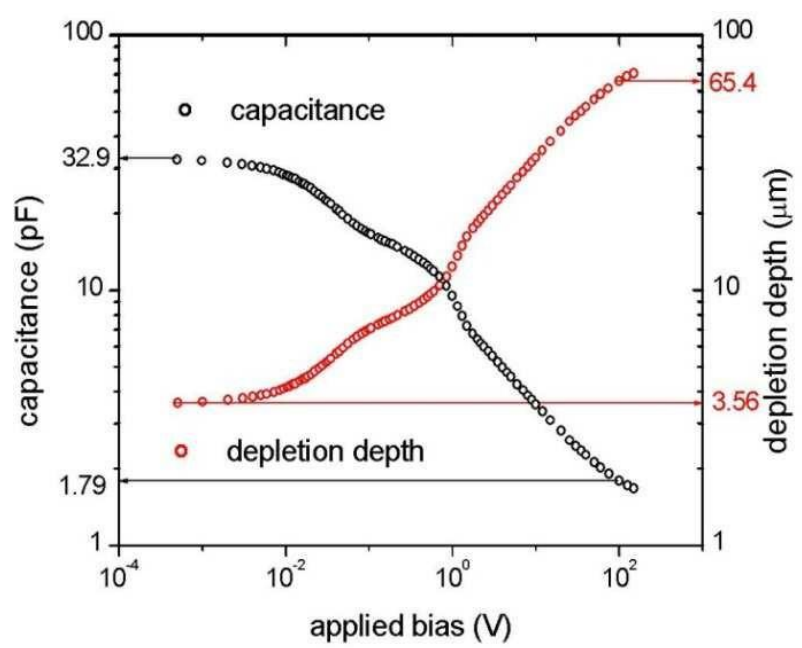


Fig 43: C-V characteristic of the S5821 diode is shown together with the calculated depletion depth as a function of applied reverse bias

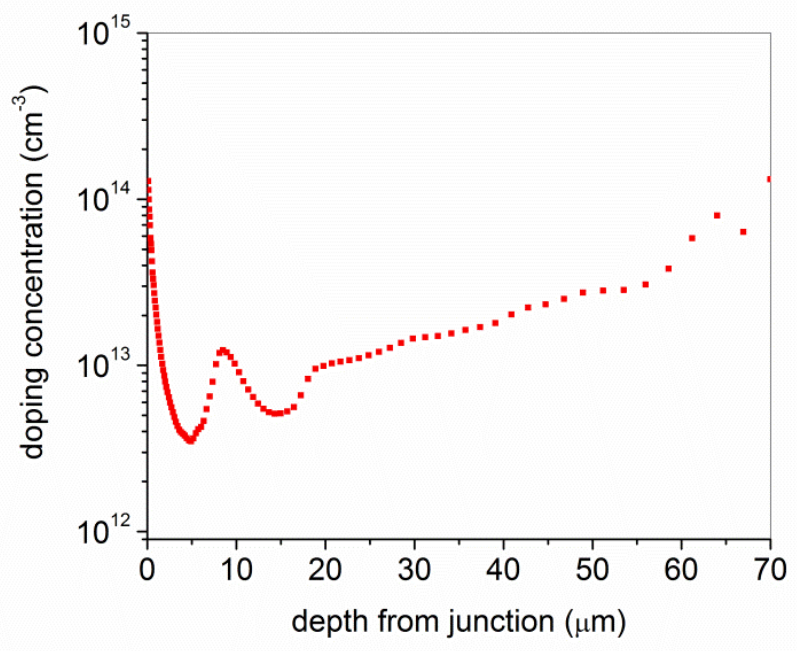

Fig 44: Calculated doping profile of the diode (S5821) using the measured C-V data 
Electron injection

1 Quiescent reverse bias $\left(V_{R}\right)$

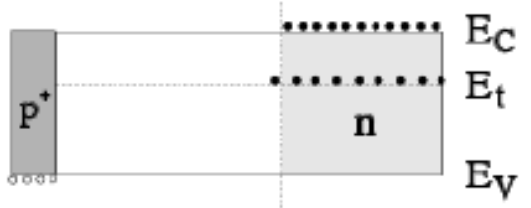

2 Majority carrier pulse $\left(V_{P}\right)$

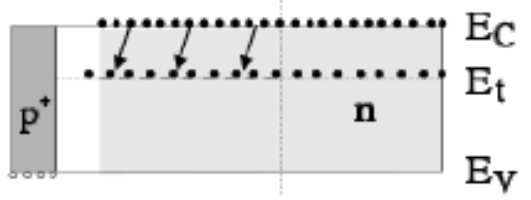

3 Thermal emission of carriers $\left(V_{R}\right)$
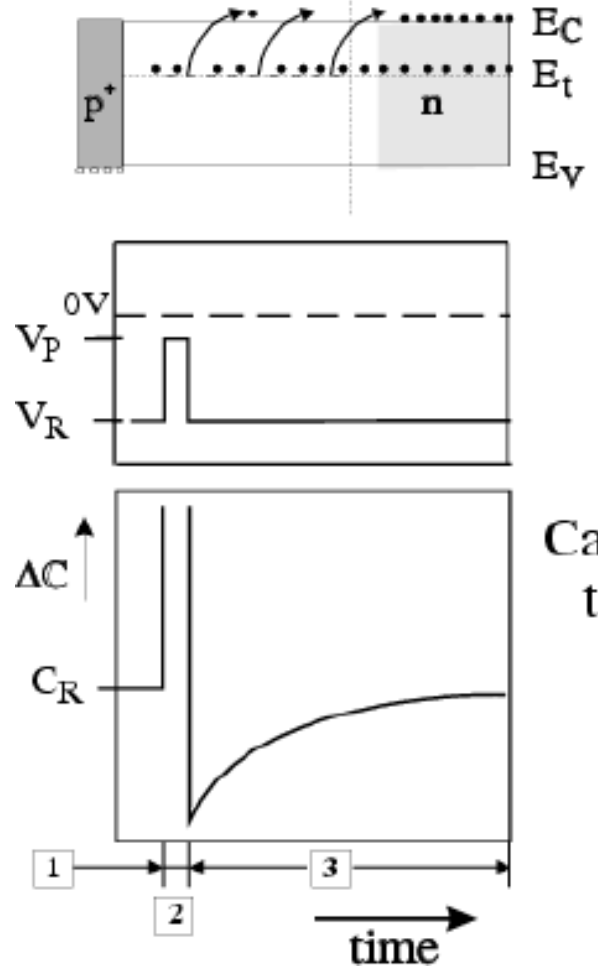

Hole/Electron injection

1 Quiescent reverse bias $\left(V_{R}\right)$

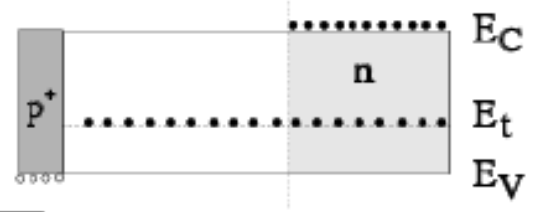

2 Injection pulse ( $V_{P}$, forward bias)

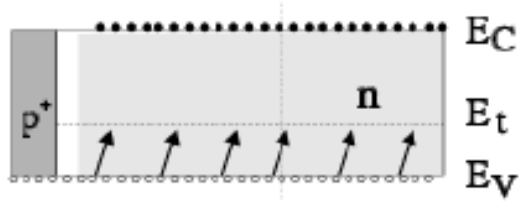

3 Thermal emission of carriers $\left(V_{R}\right)$

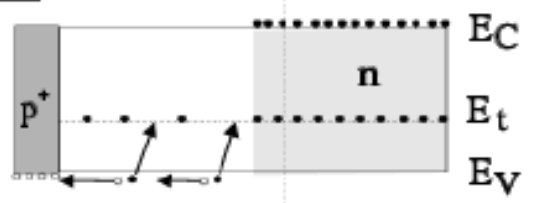
$\mathrm{E}_{\mathrm{V}}$

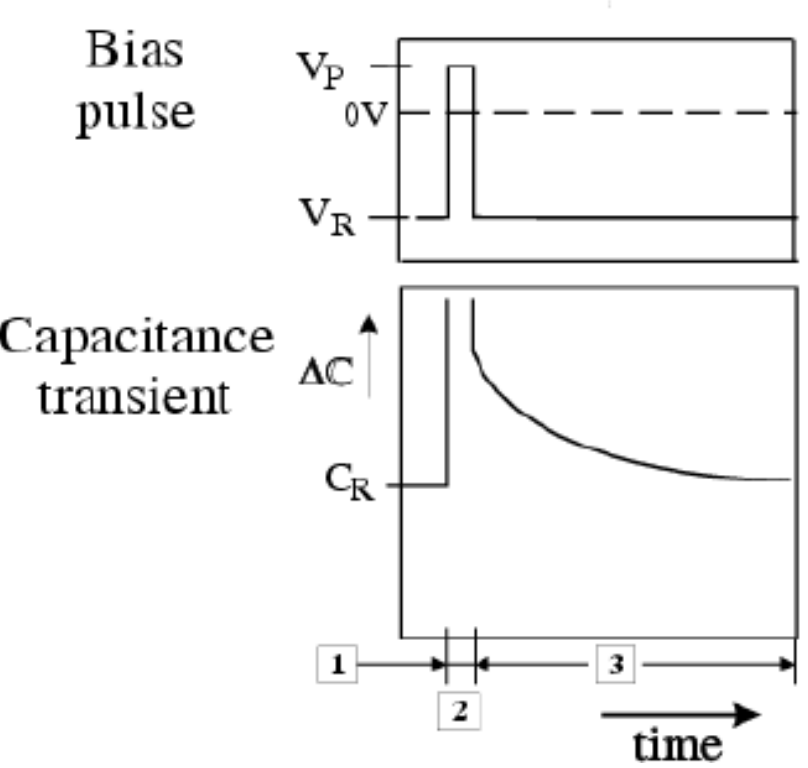

Fig 45: The principle of DLTS: (i) for electron injection and (ii) for hole/electron injection

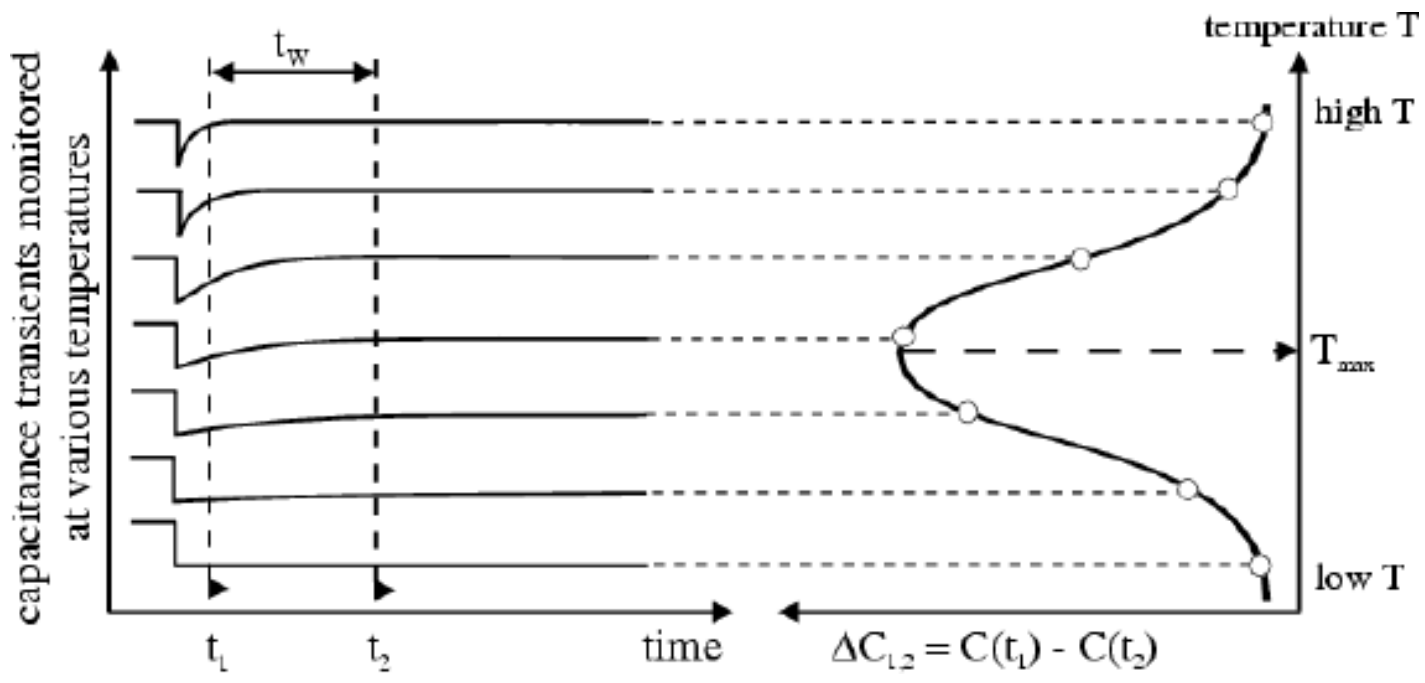


Fig 46: Processing of the recorded capacitance transients for a given rate window is shown together with the corresponding peak-shaped capacitance signal as a function of temperature.

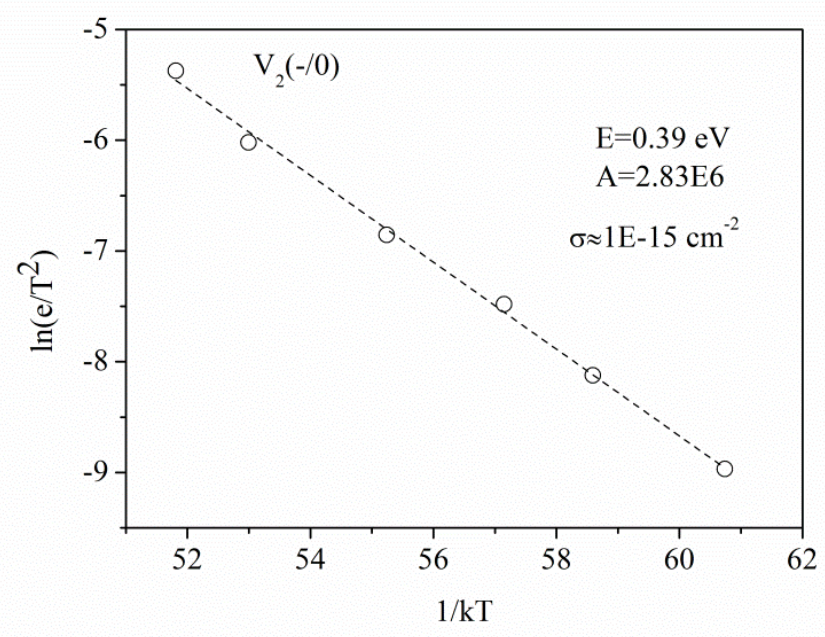

Fig 47: Arrhenius plot for the $\mathrm{V}_{2}(-/ 0)$ defect created in silicon by $1.5 \mathrm{MeV} \mathrm{Si}$ ion.

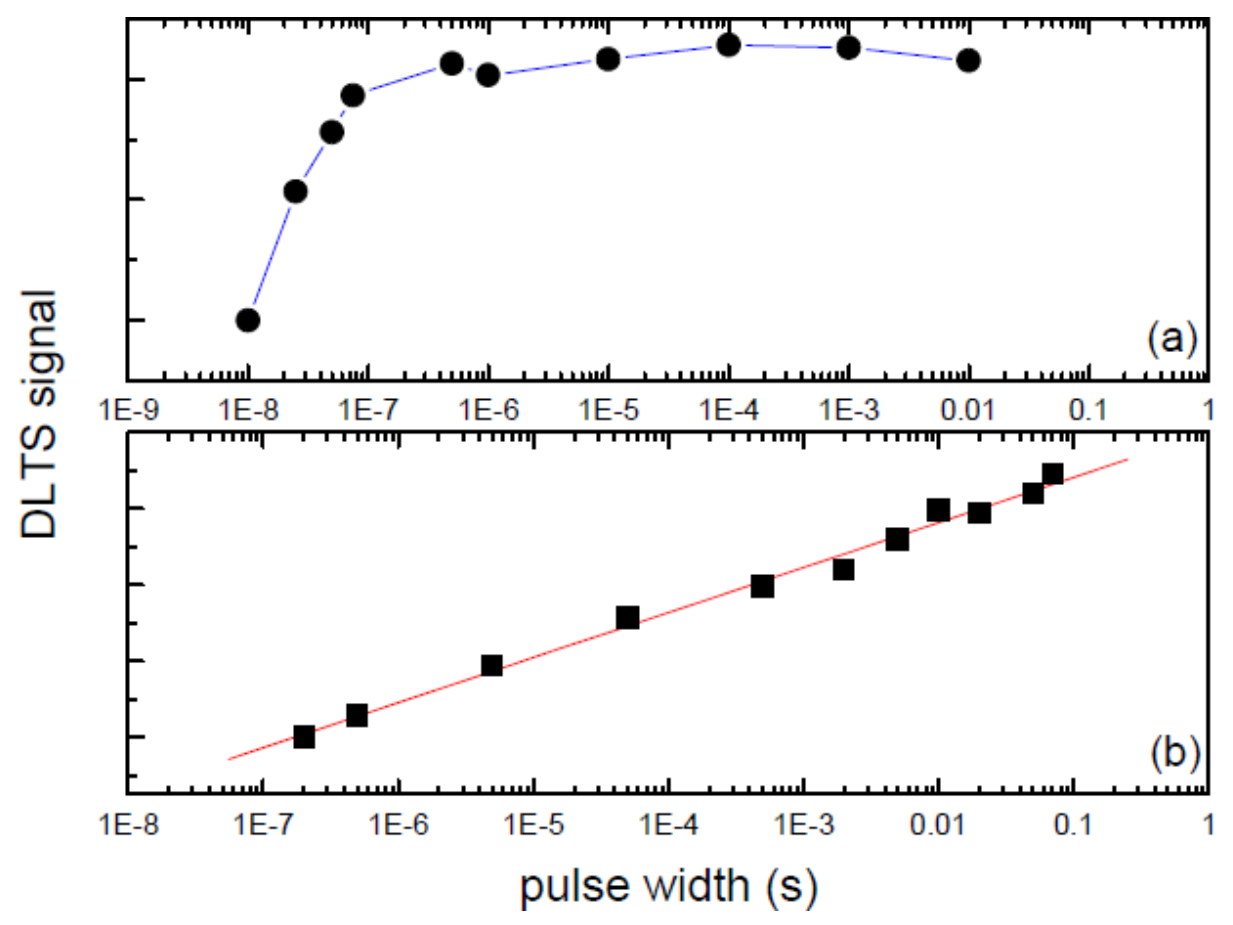

Fig 48: DLTS signal as a function of pulse width; typical for the: (a) point-like defects, (b) extended (cluster) defects

$\mathrm{x}=\mathrm{d}$, is grounded. 


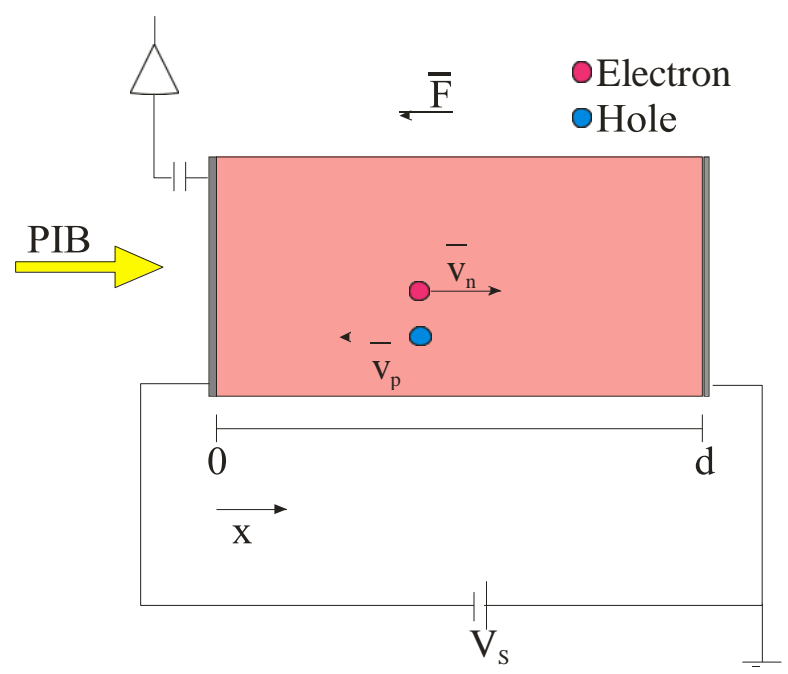

Fig. 49: Schematics of the one-dimensional geometry
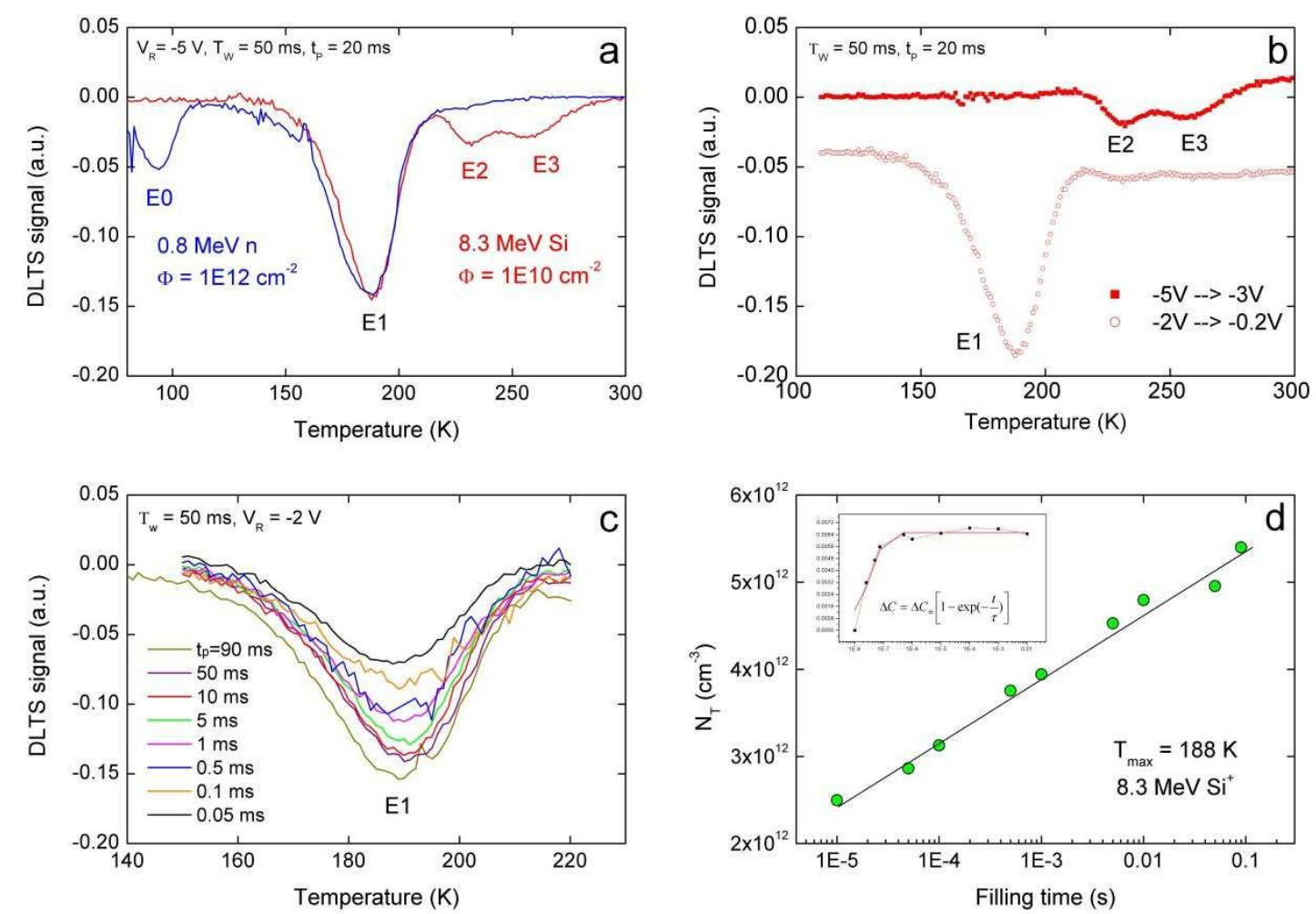

Fig. 50: Results of DLTS spectroscopy performed on implanted silicon diodes: a) normalized DLTS spectra obtained by $8.3 \mathrm{MeV}$ Si single ion implantation (red) and 0.8 $\mathrm{MeV}$ neutron irradiation (blue); b) depth profiling of the vacancy-rich surface side of the implant tail (circles) and interstitial-rich region near the implant peak (squares) - off-set spectra are shown; c) section of DLTS spectra corresponding to E1 trap formed in the implant tail as a function of the filling time (tp); d) results of carrier capture cross-section analysis of the $\mathrm{E} 1$ trap showing logarithmic behaviour typical for complex (cluster) defect structures. Inset in fig.2 (d) shows the exponential dependence of carrier capture cross section typical for point defects. 


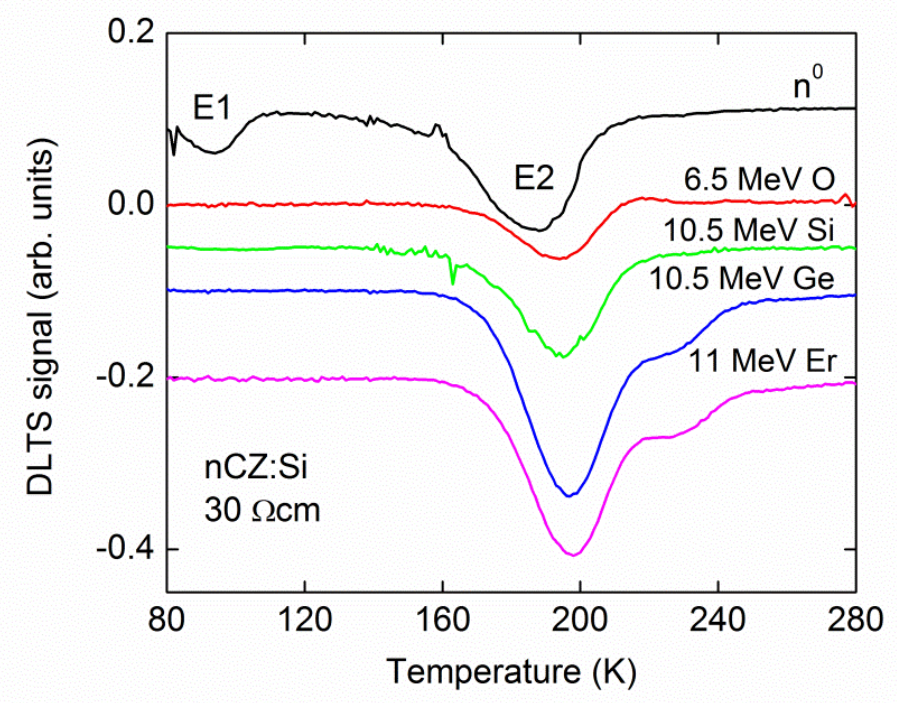

Fig. 51: DLTS spectra for all samples, fast neutron irradiated and ion implanted, measured with the emission rate $4.65 \mathrm{~s}-1$, reverse bias $-5 \mathrm{~V} \rightarrow-0.2 \mathrm{~V}$ and pulse duration $10 \mathrm{~ms}$. The spectra are vertically shifted for the clarity.

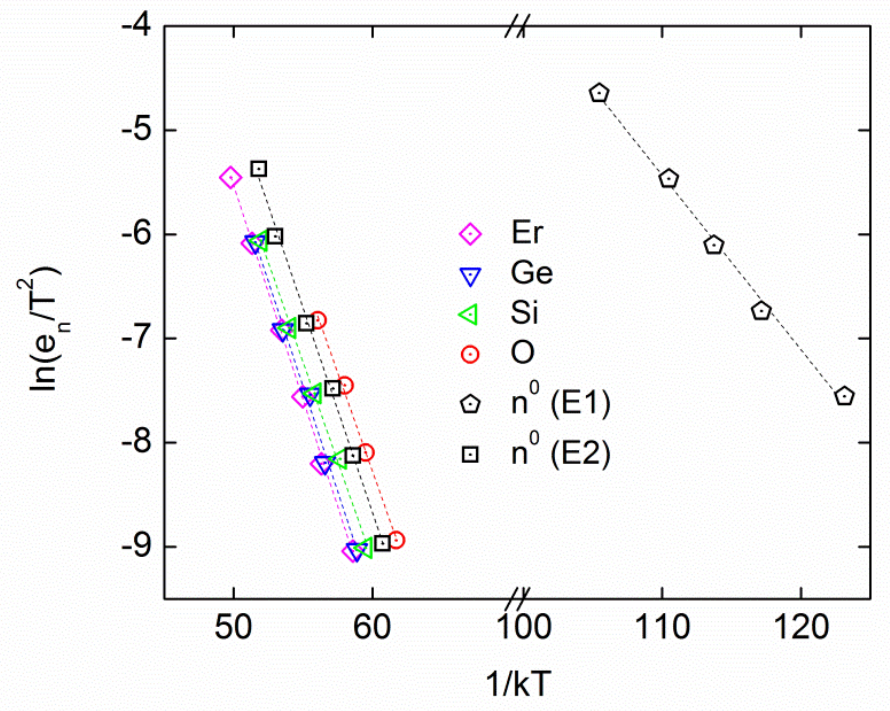

Fig.52: Arrhenius plots of T2-corrected emission rates for all observed defects. Electronic parameters derived from the plots are given in Table 2.

Table 4: Activation energies for electron emission, pre-exponential factor and the capture cross section for all observed traps derived from Arrhenius plots.

\begin{tabular}{|c|c|c|c|c|}
\hline $\begin{array}{c}\text { Damagin } \\
\mathrm{g} \\
\text { particle }\end{array}$ & $\begin{array}{l}\text { Activatio } \\
\mathrm{n} \\
\text { energy/e } \\
\mathrm{V}\end{array}$ & $\begin{array}{c}\text { Defect } \\
\text { identificatio } \\
\mathrm{n}\end{array}$ & $\begin{array}{c}\text { Pre- } \\
\text { exponenti } \\
\text { al factor }\end{array}$ & $\begin{array}{c}\text { Capture cross- } \\
\text { section }\end{array}$ \\
\hline $6.5 \mathrm{MeV} \mathrm{O}$ & $\begin{array}{c}0.38 \pm \\
0.01\end{array}$ & $\mathrm{~V}_{2}(-/ 0)$ & $\begin{array}{c}9.37 \times 10^{5} \mathrm{~s}^{-1} \\
\mathrm{~K}^{-2}\end{array}$ & $2 \times 10^{-15} \mathrm{~cm}^{2}$ \\
& $\begin{array}{c}0.39 \pm \\
\mathrm{V}_{2}(-/ 0)\end{array}$ & $\begin{array}{c}1.67 \times 10^{6} \mathrm{~s}^{-1} \\
\mathrm{~K}^{-2}\end{array}$ & $5 \times 10^{-15} \mathrm{~cm}^{2}$ \\
\hline $10.5 \mathrm{MeV} \mathrm{Si}$ & \begin{tabular}{c}
0.02 \\
\hline
\end{tabular} & &
\end{tabular}




\begin{tabular}{|c|c|c|c|c|}
\hline $10.5 \mathrm{MeV} G \mathrm{G}$ & $\begin{array}{c}0.40 \pm \\
0.02\end{array}$ & $\mathrm{~V}_{2}(-/ 0)$ & $\begin{array}{c}2.45 \times 10^{6} \mathrm{~s}^{-1} \\
\mathrm{~K}^{-2}\end{array}$ & $6 \times 10^{-15} \mathrm{~cm}^{2}$ \\
\hline $11 \mathrm{MeV}$ & $\begin{array}{c}0.41 \pm \\
\mathrm{Er}\end{array}$ & $\mathrm{V}_{2}(-/ 0)$ & $\begin{array}{c}3.37 \times 10^{6} \mathrm{~s}^{-1} \\
\mathrm{~K}^{-2}\end{array}$ & $6 \times 10^{-15} \mathrm{~cm}^{2}$ \\
\hline $\mathrm{n}^{0}(\mathrm{E} 1)$ & $\begin{array}{c}0.17 \pm \\
0.01\end{array}$ & $\mathrm{VO}(-/ 0)$ & $\begin{array}{c}4.31 \times 10^{5} \mathrm{~s}^{-1} \\
\mathrm{~K}^{-2}\end{array}$ & $2 \times 10^{-14} \mathrm{~cm}^{2}$ \\
\hline $\mathrm{n}^{0}(\mathrm{E} 2)$ & $\begin{array}{c}0.39 \pm \\
0.01\end{array}$ & $\mathrm{~V}_{2}(-/ 0)$ & $\begin{array}{c}1.55 \times 10^{6} \mathrm{~s}^{-1} \\
\mathrm{~K}^{-2}\end{array}$ & $5 \times 10^{-15} \mathrm{~cm}^{2}$ \\
& 0 & & \\
\hline
\end{tabular}

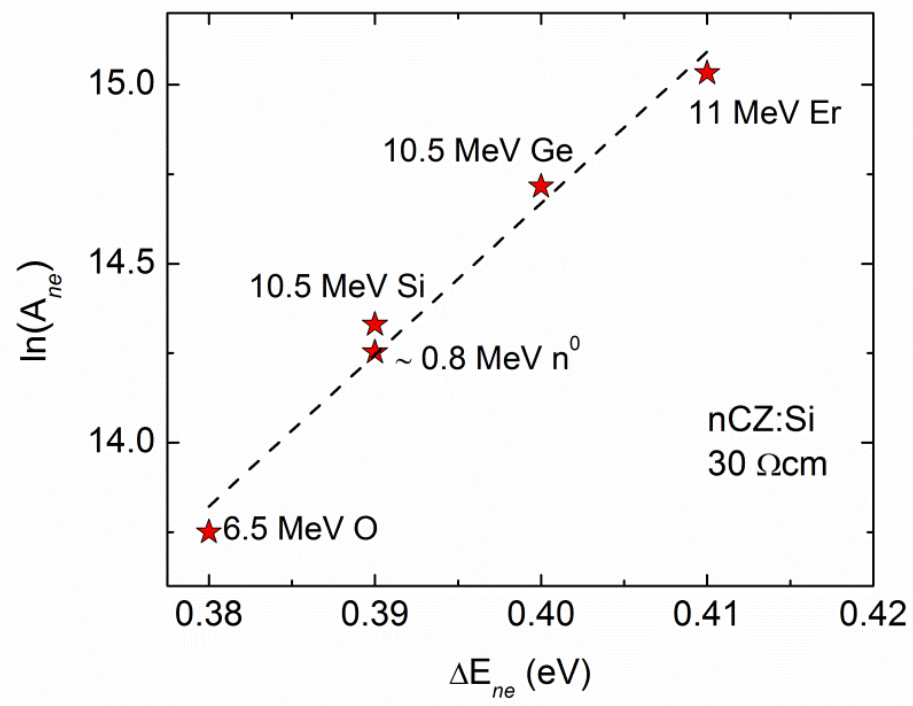

Fig. 53: The pre-exponential factor as a function of the activation energy for electron emission for the V2(-/0) defect in ion implanted $(\mathrm{O}, \mathrm{Si}, \mathrm{Ge}, \mathrm{Er})$ and fast neutron irradiated samples.

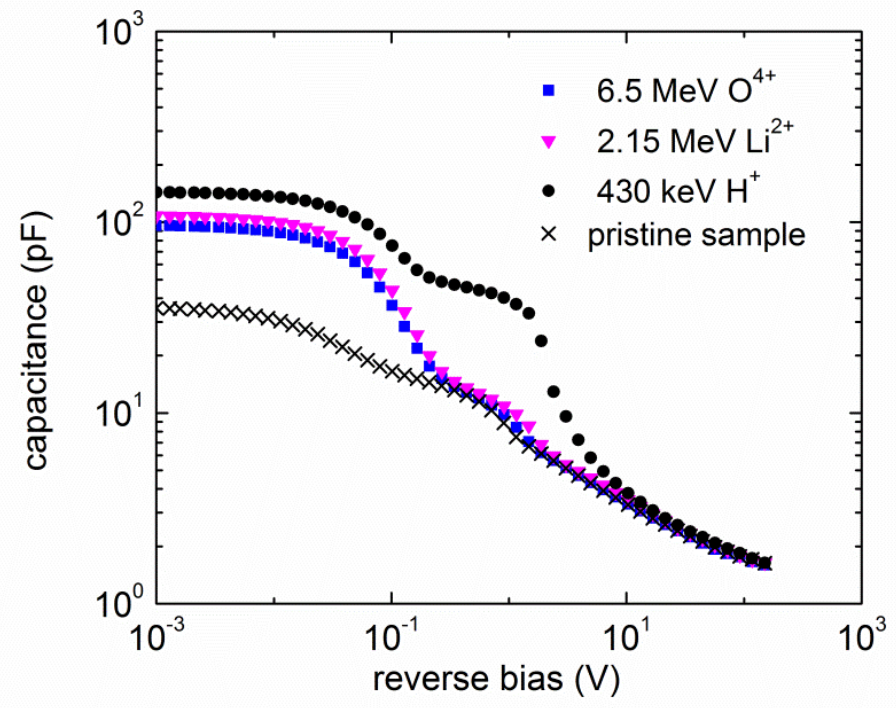

Fig.54: C-V characteristics of the unirradiated and irradiated photodiodes. Measured capacitance values at room temperature are depicted with crosses for unirradiated, with squares for oxygen, with triangles for lithium and with circles for proton irradiated devices. 


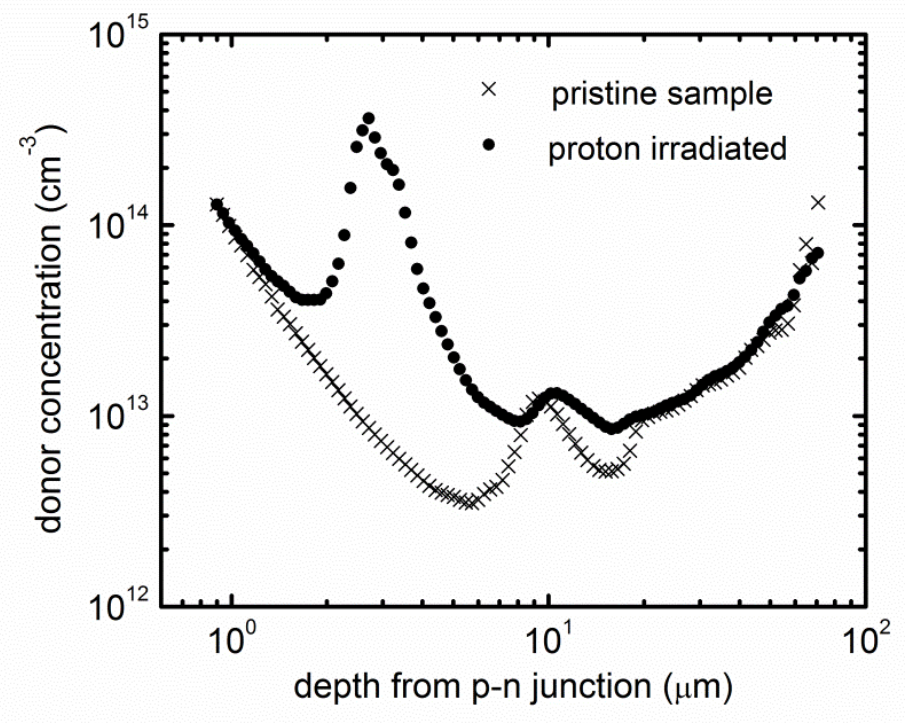

Fig.55: Calculated donor concentration depth profiles in the matrix of the virgin photodiode (initial state) and the photodiode irradiated with $430 \mathrm{keV}$ protons.

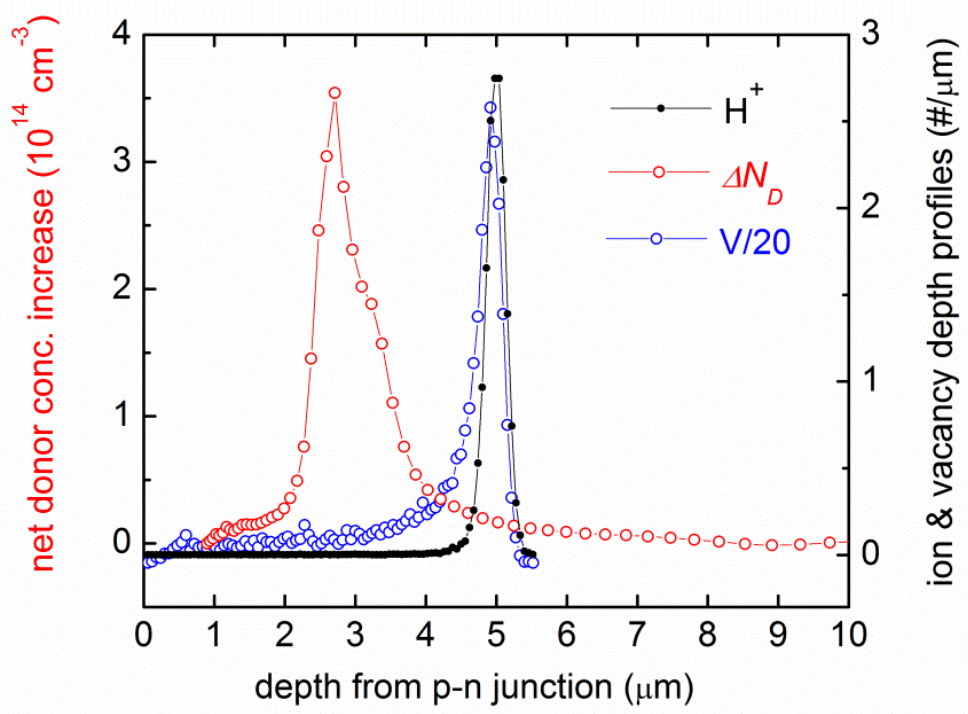

Fig.56: TRIM calculated implanted hydrogen $\left(\mathrm{H}^{+}\right)$and vacancy production rate $(\mathrm{V}$; divided by a factor of 20) depth profiles are shown together with the hydrogen irradiation-related defect concentration (NT) in the subsurface region of the photodiode irradiated with $2.45 \cdot 10^{11} \mathrm{~cm}^{-2}$ fluence of $430 \mathrm{keV} \mathrm{H}^{+}$ions. 


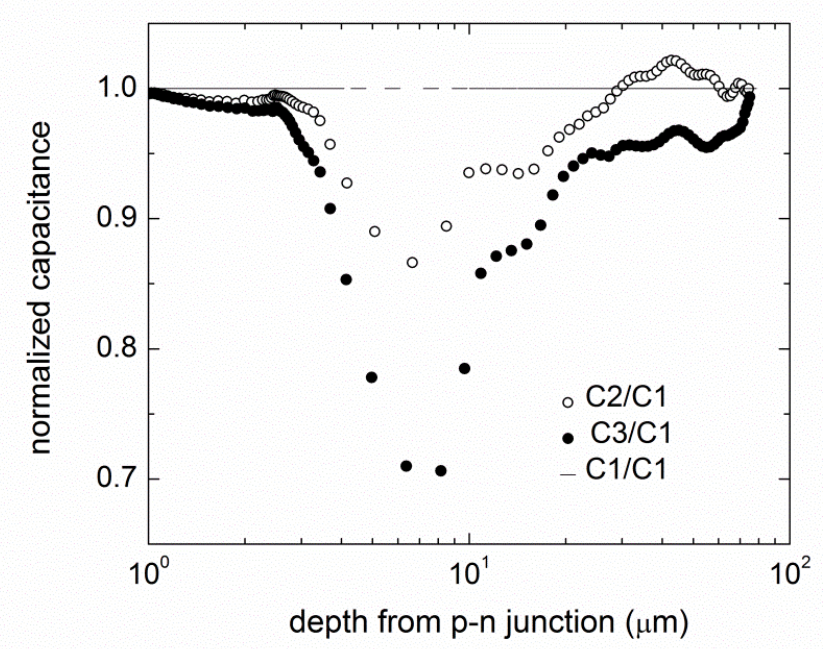

Fig.57: Normalized capacitance depth profiles of the photodiode irradiated with $430 \mathrm{keV}$ protons during process of self-annealing at the room temperature. The line represents normalizing capacitance, C1, taken 2 weeks after irradiation. C2 and C3 profiles are obtained 6 and 30 weeks after the irradiation, respectively.

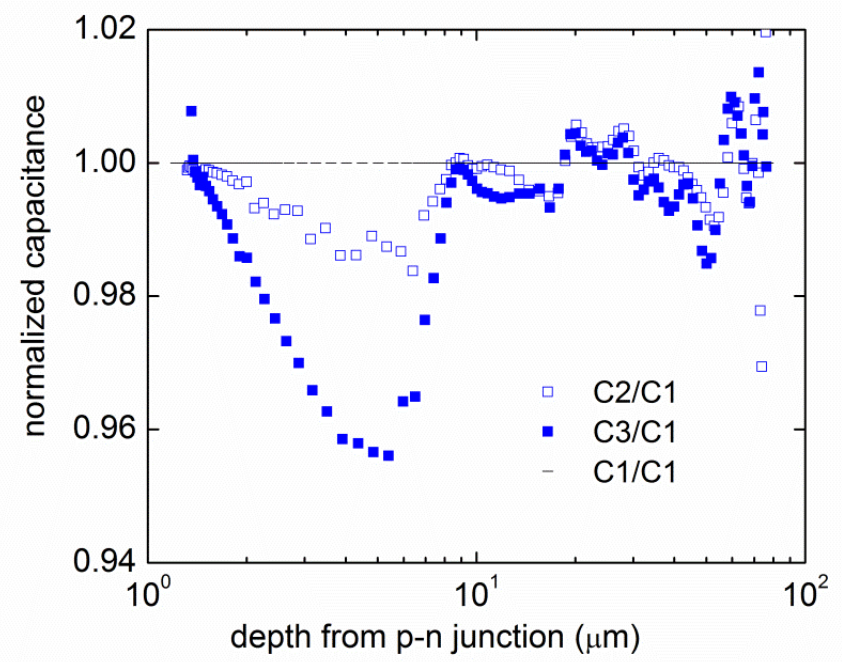

Fig.58: The normalized capacitance profile during the self-annealing of the photodiode irradiated with $6.5 \mathrm{MeV}$ oxygen ions. The black line corresponds to the maximum capacitance, C1, measured 2 weeks after the irradiation. Hollow and filled squares depict normalized C-V measurements C2 and C3. 


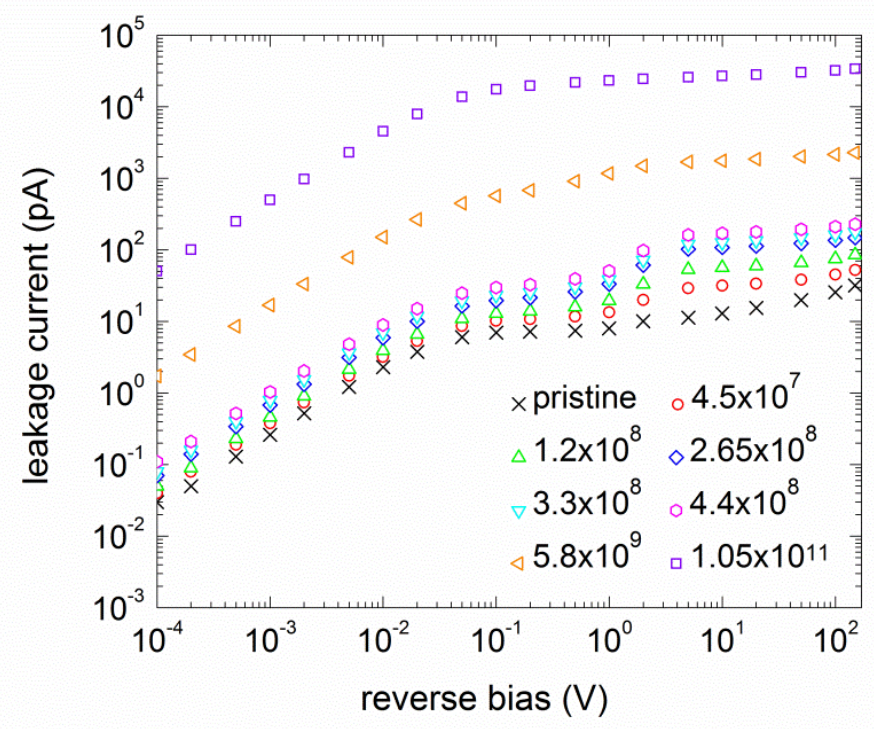

Fig. 59: Experimental data for the leakage current of a photodiode irradiated with 5.5 $\mathrm{MeV}$ alpha particles as a function of applied reverse bias for different alpha particle fluences.

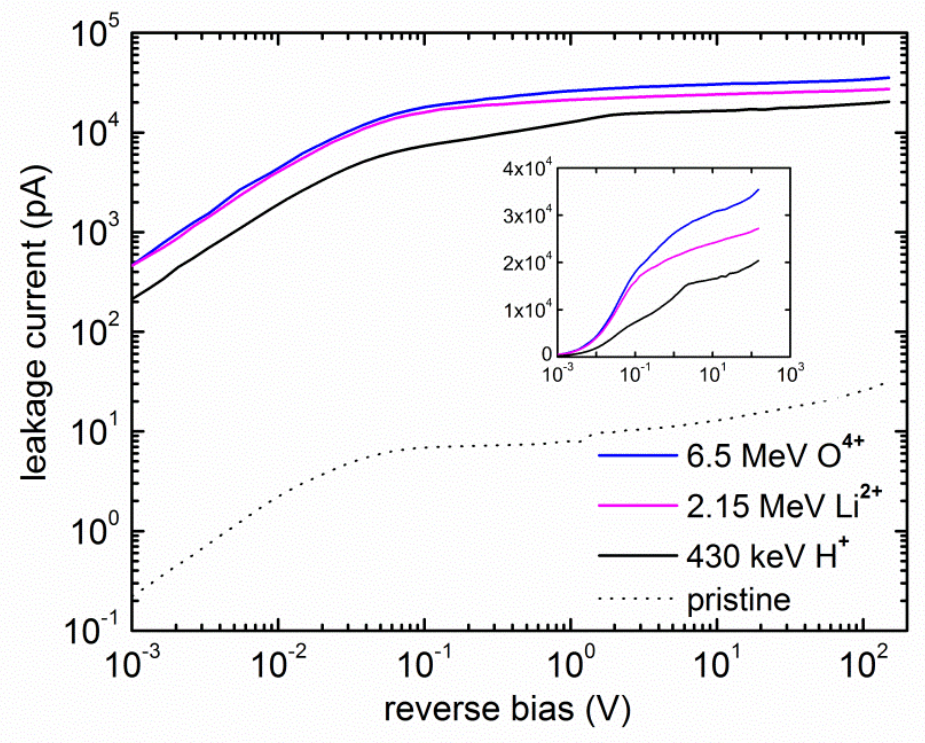

Fig.60: NIEL scaled (to the proton value) I-V characteristics of the irradiated photodiodes in the bias range relevant for ion beam analysis (IBA) techniques. The main graph depicts current values on the logarithmic scale. The inset graph depicts the differences between the scaled leakage currents on linear scale. Blue, magenta, blacksolid and black-dotted curves correspond to the oxygen-, lithium-, proton-irradiated, and unirradiated photodiodes, respectively. 